\title{
RAMAN STUDIES OF HEAVILY CARBON \\ DOPED GaAs
}

by

MOONSUK SEON, B.S., M.S.

\section{A DISSERTATION}

IN

\section{PHYSICS}

Submitted to the Graduate Faculty

of Texas Tech University in

Partial Fulfillment of

the Requirements for

the Degree of

DOCTOR OF PHILOSOPHY

Approved

Accepted

Dean of the Graduate S $₫$ hool

August, 1999 


\section{ACKNOWI.EDGEMENTS}

I still remember those many nights. On the way walking back home. I used 11 raise my eyes, look up the dark sky, stare twinkling stars and sang a song. " l'ou light up my life." While I sang the song, I was thinking of many people. Those who filled m mind when I felt empty, those who helped me to find my way whenever I was lost. and those who gave me their hand when I fell down.

At this moment, what is important to me is not the Ph.D. title. What is important is the fact that I have learned academically and personally how to persevere. and finish what I start. I now have the courage to manage research in any unfamiliar research environment. I deeply thank many people who helped me to overcome a feeling of despair. a temptation to quit, and lack of self-confidence. I can stand up again because I saw the faces of those who filled my mind, guided my path, and gave me their hand. This place where I have stayed since 1995 will be remembered as a beautiful place because of them, regardless of its sweltering temperatures, dusty winds, and low humidity.

I would like to thank my friends for their support. Kyung-Hee Lee. Jung-Eun Jang, Hee-Su Kim, Kyung-Mi Kang and Yun-Kyung Lee. They showed long lasting and strong friendship in Korea. In-Hee Baik, Mi-Seon Park. Dae-Hee Lee, Woo-Rahn Lee. Sun-Hee Kim, Brigette Hayes and Seul-Hee Yoo made me feel at home here with their warm hearts and kind actions.

I also would like to thank Kim Zinsmeyer, Lee Burnside, Richard Hernandez, and Sarah Stubbs for their help they offered me whenever I needed it. I am grateful to Dr. Charles Myles and Dr. Shubhra Gangopadhyay for their concern for me. I am also 
grateful to Dr. Charles Mỳles, Dr. Roger Lichti and Dr. Henryk Temkin for serving on my dissertation committee.

I deeply appreciate Dr. Ta-Ryeong Park. He was here when I had a hard time. He helped and encouraged me when I took my qualifying test. He gave me good advice for my research. He was one of main driving forces for silicon-germanium multi-quantum well project. I would like to thank Dr. Toni Sauncy and Dr. Tim Dallas. They helped me to become accustomed to the laboratory environment. When I first joined Dr. Holtz group in May 1995, I almost had no experience in a research level-laboratory. Everything was new to me. They showed me how things can be done. I also thank Dr. Tatayna Prokofyeva for her company. She is a mentally strong woman. like Dr. Toni Sauncy. and their strength encouraged me to break through any obstacles.

Whenever I think of my family. I cannot stop smiling. I love all of my family members. and feel their love toward me. I am proud of my family because they have a right and respectful point of view of society, the world. and right and wrong. I could concentrate on my studies here due to their existence. especially my elder sister. Misug Seon. who stayed in Lubbock with me for a year. She fed my body and soul. I respect and thank her for such generosity and kindness.

To me. May of 1995 is a very important time because I joined Dr. Holtz ${ }^{\circ}$ group at that time. My memory of the day when he offered me a research assistant position for the summer is picturesquely clear. I remember how he called my name with a warm smile. how I moved toward him with hopping steps. and how I accepted his offer with excitement. I always wanted to be a good student. It has been my goal since I joined Dr. Holtz s group. even though I could not achieve it up to now. I deeply respect Dr. Holtz as 
a physicist, mentor, and a human being. He has given me a standard to strive for in $\mathrm{m}$ ! life ahead. 


\section{TABLES OF (ONTENTS}

ACKNOWLEDGEMFNTS

ABSTRACT

LIST OF TABLES

LIST OF FIGURES

I. MOTIVATION

II. BACKGROUND

2.1. Introduction

2.2. Local vibrational modes

2.3. Vibrations in crystals

2.3.1. Linear diatomic chain 15

2.3.2. Vibrational structure of GaAs

2.4. Longitudinal phonon-plasmon coupled modes

2.4.1. Low damping and $\vec{k}=0$

2.4.2. Low damping and $\vec{k} \neq 0$

2.4.3. High damping

2.5. Raman spectroscopy

2.6. Photoluminescence

2.7. Annealing

III. EXPERIMENTAL DETAILS 49

3.1. Sample information t9

3.2. Photoluminescence 50

3.3. Raman spectroscopy 53

3.4. Annealing 57

IV. PHOTOLUMINESCENCE OF HEAVILY CARBON DOPED GAAS

4.1 Photoluminescence from unannealed GaAs: $\mathrm{C}$ 59

4.2 Photoluminescence from annealed GaAs: $\mathrm{C}$

4.3 Summary 69

V. RAMAN SPECTROSCOPY OF HEAVILY CARBON DOPED GAAS 70)

5.1 The $C_{A}$, local vibrational mode 70

5.2 Longitudinal optic-plasmon coupled band 75

5.3 Discussion of the LO mode intensity

5.4 Raman Spectroscopy from annealed GaAs:C

$\begin{array}{ll}5.5 \text { Summary } & 107\end{array}$ 
VI. SUMMARY

REFERENCES

114 


\section{ABSTRACT}

Optical measurements, mainly Raman spectroscopy. are used to study GaAs heavily doped with carbon. Hole concentration in these samples ranges from $2.3 \times 10^{19}$ to $1 \times 10^{20} \mathrm{~cm}^{-3}$. Three main Raman features are investigated: the longitudinal-optic (LO) phonon mode. the substitutional carbon-at-arsenic local vibrational mode $\left(C_{A s} L V M\right)$. and the coupled plasmon-LO phonon mode (LOPC). $\mathrm{C}_{\mathrm{As}}$ LVM intensity is directly proportional to carrier concentration. This implies that $\mathrm{C}_{\mathrm{As}} \mathrm{LVM}$ intensity is a good carrier density indicator, even though its practical use is limited by its weakness. Only one phonon-like coupled mode is observed due to the large plasmon damping and high effective carrier masses. The coupled mode is seen to systematically red shift as $p$ increases even though the peak width of the mode stays constant. This behavior is described by a model which includes the effects of high hole concentrations on the dielectric function and an additional shift in the LO phonon we attribute to carbon size effect. Interestingly, the local mode intensity shows good correlation with that of LOPC mode as a function of $p$. Based on these results, the intensity of LOPC to that of the LO phonon is determined to be a good indicator of the carrier concentration.

$\mathrm{I}_{\text {LOPC }} / \mathrm{I}_{\text {LO }}$ decreases upon annealing, implying $p$ reduction. Simultaneously, two peaks around 1375 and $1600 \mathrm{~cm}^{-1}$ appear in all the annealed samples. The two peaks are assigned to carbon precipitates. From the observation, it is believed that carbon precipitates into a nanocrystalline graphite phase upon annealing. The crystal size of the carbon precipitates was estimated from the peak intensity ratio. 
Emission due to a conduction band to acceptor level transition (e.A) was observed from photoluminescence (PL) spectra. The band redshifts as carrier concentration increases due to band gap shrinkage. PL intensity of the (e,A) transition in annealed samples is drastically decreased. Carrier concentration reduction and the formation of nonradiative recombination centers are suggested as the cause of the behavior, and carbon precipitates observed in Raman spectra are suggested as the possible nonradiative recombination centers. 


\section{LIST OF TABLES}

2.1 Character table and basis functions for carbon at arsenic LVM.

2.2. Atomic displacement Raman selection rules for backscattering from the (001) surface.

2.3 Surface field, linear $\mathrm{k}$, and atomic displacement Raman selection rules for backscattering from the (001) surface.

3.1 Heavily carbon doped GaAs sample information

3.2 List of parameters used for Eq. 2.24 and Eq. 5.6. 


\section{LIST OF FIGLRES}

2.1 Local vibrational mode frequencies in GaAs due to impuritics with different masses.

2.2 Crystal structure of GaAs and its crystallographic axes.

2.3 Carbon at arsenic LVM with $\Gamma_{15}$ symmetry of the $T_{d}$ group.

2.4. Dispersion curves of (a) 1-dimensional diatomic. (b) 3-dimensional diatomic systems and (c) GaAs.

2.5 Calculated plasmon-phonon mode frequencies as a function of free carrier concentration for Raman scattering from CiaAs at $51+.5 \mathrm{~nm}$. The solid lines correspond to the bulk LO and TO modes. The calculations ate been corrected for finite k-rector and non-parabolic effective mass effects [136].

2.6 Dispersion of the peaks in the Raman spectra of coupled plasmon-LO phonon modes of $n$-Ga.As. The solid and dashed lines are calculated by Abstreiter et al. [49], the dots represent the experimental results.

2.7 Schematic diagrams of the interband and intraband electronic transitions which play a role in (a) two-band and (b) three-band Raman processes. (c) Schematic diagrams of the additional interband and intraband electronic transitions which playa role in scattering processes which take place in an $n$-type semiconductors [69]. The numbers indicate the order of the electronic transitions. C. $C_{1}$ and $C_{2}\left(V . V_{1}\right.$, and $\left.V_{2}\right)$ are conduction (valence) bands.

2.8 Energy level diagram for rotational. vibrational and electrical levels.

2.9 Photoluminescence spectra of (a) pure GaAs [90] and (b) n-type GaAs:Te and p-type GaAs:Zn [94] at $77 \mathrm{~K}$.

2.10 Di-carbon $\left(C_{i}-C_{A s}\right)$ defect structure proposed by Wagner ct al. [105].

3.1 Photoluminescence setup.

3.2 Micro-Raman setup for Raman measurements.

$3.3 \quad$ Annealing sistem. 
4.1 Photoluminescence spectra from unannealed GaAs: (' samples for different carrier concentrations at $77 \mathrm{~K}$. ise $=488 \mathrm{~nm}$.

4.2 Position of the photoluminescence emission peak at $77 \mathrm{~K}$.

4.3 A schematic of the band structure in both undoped and heavily $p$-type doped GaAs [76].

4.4 Optical-micrographs from unannealed (a) $p=4.3 \times 10^{19} \mathrm{~cm}^{-3}$ and (b) $p=$ $1 \times 10^{211} \mathrm{~cm}^{-3}$ samples, and annealed (c) $p=4.3 \times 10^{19} \mathrm{~cm}^{-3}$. (d) $p=1 \times$ $10^{20} \mathrm{~cm}^{-3}$ samples. Theses are taken from capped area. Annealing conditions are $850^{\circ} \mathrm{C}, 1$ hour. (e) Taken from uncapped annealed $p=1 \times$ $10^{20} \mathrm{~cm}^{-3}$ sample.

4.5 Photoluminescence spectra from annealed $p=1 \times 10^{-11} \mathrm{~cm}^{-3}$ GaAs:C samples for different annealing conditions at $77 \mathrm{~K}$. $\lambda_{\text {cse }}=488 \mathrm{~nm}$.

5.1 Room temperature Raman spectra in the $C_{A}$, LVM range. $\lambda_{\text {exc }}=+57.9$ nm.

5.2 $\mathrm{C}_{\mathrm{As}}$ LVM intensity normalized by $2 \mathrm{TO}$ overtone intensity in these samples studied. $\lambda_{\mathrm{exc}}=457.9 \mathrm{~nm}$.

5.3 Compilation of relative intensities of the $\mathrm{C}_{\mathrm{A}}$ LVM (gray squares, right hand scale) and the LOPC band (filled circles. left-hand scale) to the LO mode from this study. Other data are for GaAs doped with Be from Refs. 52, 62, 65 and Ge, Ref. 67. The dashed line corresponds to direct dependence between the relative intensities and the carrier concentration.

5.4 Room-temperature, symmetry allowed Raman spectra of carbon doped GaAs samples studied here, spanning the range of hole concentrations studied. Data are normalized with respect to LOPC band intensity. A systematic change in relative intensities and red shifting are observed.

5.5 Polarized Raman spectra of the (a) most lightly and (b) most heavily doped samples studied here. Line shapes and positions in (b) do not vary significantly between the different spectra, which is different in (a).

5.6 The Raman scattering rate calculated by Eq. 5.6. Spectra are grouped by the same plasmon damping constant.

5.7 The Raman scattering rate calculated by Eq. 5.6. Spectra are grouped by the same plasmon frequency. 
5.8 LO phonon (a) and LOPC band (b) positions versus hole concentration (457.9 $\mathrm{nm}$ excitation). The solid squares represent our data while data for $\mathrm{Zn}$ and $\mathrm{Be}$ doped samples are included as diamonds and circles, respectively. (a) The solid line is a linear fit to the GaAs:C data. (b) The upper curve is from Eq. 2.24 with the parameters listed in Table 5.1. The lower curve includes the optic-phonon softening implied by the data in (a).

5.9 The Fermi level at the epilayer is very close to or under the top of valence band, and is pinned at the midgap at the surface. The Fermi level should be a straight line under equilibrium condition. resulting in the depletion region near surface.

5.10 Room-temperature Raman spectra taken from (a) unannealed and (b) (g) annealed $p=4.6 \times 10^{19} \mathrm{~cm}^{-3}$ samples. Samples are annealed between $350^{\circ} \mathrm{C} \sim 850^{\circ} \mathrm{C}$ during 1 hour. Peaks with * sign are Ar plasma lines.

5.11 Raman spectra taken from (a) unannealed and (b) annealed bulk GaAs and (c) unannealed and (d) annealed $p=4.6 \times 10^{19} \mathrm{~cm}^{-3}$ samples. Annealing condition is $850^{\circ} \mathrm{C}$ and 1 hour.

5.12 Raman spectra taken from annealed $p=4.6 \times 10^{19} \mathrm{~cm}^{-3}$ samples. Samples are annealed 1 hour at different temperatures. Spectra are fitted with $2 \sim 4$ peaks to calculate $\mathrm{I}_{\mathrm{LOPC}} / \mathrm{I}_{\mathrm{LO}}$ ratio.

5.13 Raman spectra taken (a) unannealed and (b) annealed $p=1 \times 10^{20} \mathrm{~cm}^{-3}$ samples. $\mathrm{I}_{\mathrm{LOPC}} / \mathrm{I}_{\mathrm{LO}}$ and $\mathrm{I}_{\mathrm{LVM}} / \mathrm{I}_{\mathrm{LO}}$ decrease upon annealing.

5.14 $\mathrm{I}_{\text {LOPC }} / \mathrm{I}_{\text {LO }}$ calculated from unannealed and annealed samples.

5.15 Normalized $\mathrm{I}_{\text {LOPC }} / \mathrm{I}_{\text {LO }}$ with respect to that of unannealed samples.

5.16 Raman spectra taken from $p=1 \times 10^{20} \mathrm{~cm}^{-3}$ sample at different spot. Sample is annealed at $850^{\circ} \mathrm{C}, 1$ hour. 


\section{CHAPTER I}

\section{MOTIVATION}

Impurities are intentionally introduced into a semiconductor to modify its electronic structure. As a result, the transport and optical properties of the semiconductors are altered. The mobility, the constant of proportionality between carrier drift velocity and electric field applied, is a central parameter in characterizing electron and hole transport due to drift. The mobility plays a key role in characterizing the performance of many devices [1]. At low doping concentration. the carrier mobility is independent of the doping concentration. For high doping concentration, the mobility monotonically decreases with increasing donor or acceptor concentrations. This is due to increased ionized impurity scattering [1]. The band structure is affected by doping due to the interaction between carriers and ionized impurities. Many calculations have been performed to calculate the band structure upon doping [2-6]. The main effects of heavy doping on band structure are bandtail and band gap shrinkage. The donor (acceptor) impurities make levels below conduction band (above valence band) [7]. The donor level is one binding or ionization energy below the conduction band, and similarly the acceptor level is one binding energy above the valence band. As the impurity concentration is increased, the electron wave functions of the impurity atoms begin to overlap. An overlap of wave functions is an interaction that changes slightly the potential of each level. resulting in the formation of a band of states [8]. As the impurity concentration is increased further, the impurity band broadens and eventually merges with the nearest intrinsic band. Impurities induce band tails in the density of states by forming a band of 
impurity states as mentioned above. and by perturbing the band edge via deformation potential and Coulomb interactions [9]. Even though impurities alter electronic levels of the to host material through chemical incorporation, the difference between the effect of different impurities on electronic levels is not drastic.

Studies of lattice vibrations can give information about crystalline structure with the length scale varying over an enormous range. The coherence length of lattice vibrations is in micrometer range, which is enormously long compared to atomic level distance. Therefore, any disturbance of structure in this range can influence the lattice vibration peak position and shape. The free electrons (or holes) present due to doping constitute a plasma. In polar semiconductors the longitudinal-optical (LO) phonon possesses a strong oscillating dipole field. This polarization field of LO phonons which has information on long-range structure interacts with the plasma oscillations of free carriers. The interaction produces the LO-phonon-plasmon coupled (LOPC) mode. Therefore. the LOPC mode is relevant to long-range ordering of the material and electronic properties through electron-phonon interactions. If the mass of a substitutional impurity is smaller than that of the host atoms. the eigenvector of the vibrational mode does not have a sinusoidal or wavelike dependence in space but is strongly peaked at the impurity atom and falls off rapidly one or two lattice sites away. This results in a local vibrational mode (LVM). Local modes are on the atomic-molecular size scale. even when the electronic levels associated with the impurities are strongly coupled. The frequency and polarization properties of LVMs offer information on impurity species and substitutional site geometry because LVMs are very sensitive to substitutional mass and 
the nearest-neighbor lattice structure. These can be measured by infrared absorption and Raman scattering.

As discussed above, lattice vibrations offer information on crystalline quality wer huge range: from micrometer to angstrom. They also render information on clectronic properties through interaction with free carriers. Due to these advantages. lattice vibrations are widely used to investigate properties of materials. I review here several such effects. I choose experiments to review which were conducted by my self or others in my research group. but which were not part of this dissertation. For example. the lattice vibrational modes in Ge/Si muli-quantum wells (NQW's) are used to access the information on well thickness and interface roughness [10]. In $.11(1 \mathrm{~W}$ and superlattice structures. the Brillouin zone is folded because the periodicity is changed. Therefore. vibrational modes are active in Raman scattering which can not be observed in the bulk counterpart. The lattice vibration mode shift is related to well thickness. For perfectly sharp interfaces, a narrow interface vibration line should appear. From a rougher interface, the line becomes wider because the contributions from interface regions of different widths and/or compositions should get different weight [11]. Information on electronic bands can also be obtained by observing the resonance in vibrational modes. As an example. the $E_{1}$ energy in $\mathrm{Ge}$ well of $\mathrm{Si} / \mathrm{Ge} \mathrm{MQW}$ was obtained from the resonance of Ge related vibrational modes [10]. The scattering cross section contains, at least in principle, information about the medium like electron-phonon interaction. electron-radiation interaction and the electron band structure besides phonon energies [12]. However, it is usually impossible to extract this information because of the summation over many intermediate states involved. This becomes feasible if only one or 
a small number of intermediate states make the dominant contribution. One wa! to achieve this is by tuning the incident laser into resonance with a strong interband transition, or vice versa. In our $\mathrm{Si} / \mathrm{Ge} \mathrm{MWQ}$ study mentioned above. various presisures were applied to tune the Ge interband transition to incident laser energies.

Lattice vibrations, which are sensitive to short-range order as well as long-range structure, are also used to map the strain in the epilayer. Under stress, there are changes in the spring constant (and hence frequencies) which characterizes the phonon dispersion of solid. As an example, strain maps are attained for silicon of a patterned structure [13] and GaN on sapphire substrate [14] by analyzing lattice vibration frequency shift and/or broadening of the vibration modes.

Lattice vibrations are also used to study alloy materials, like AlGaAs and GaAsN. For AlGaAs alloy, as Al content increases, GaAs-like and AlAs-like vibrational modes show systematic shift and change in linewidth. These modes are used to study the pressure effect on the alloy [15]. For GaAsN alloy, the redshift in the GaAs-like LOphonon mode and the blue shift in GaN-like LO-phonon mode are observed and they are attributed to strain and alloying [16]. In the study, GaAs-like second-order feature is seen to broaden slightly and diminish in intensity with increasing nitrogen incorporation. These results are attributed to a weak breakdown in the zincblende crystal long-range order. The LOPC mode was used to identify whether the as grown layer is doped or not [14]. As described above. lattice vibrations are used heavily to characterize materials because they render enormous range of information. In this dissertation, those are used to investigate very heavily carbon doped GaAs. 
Most of electronic devices are made from the combination of diodes and transistors. The diodes and transistors are based on $p-n$ junction $[17] . A p-n$ junction is a single semiconductor crystal in which a region of $p$-type material is adjacent to one of $n$ type material. Depending on the type of majority carrier in the semiconductor. electron or hole. semiconductors are either $n$ - or $p$-type. These $n$ - or $p$-type semiconductors are made by intentionally introducing impurities into the host semiconductors. This process is called as doping. Doping modifies the band structure [18]. density of states. electrical and optical properties of semiconductors [18. 19]. as mentioned above. and introduces free carriers into the host material. To make devices perform as designed, very careful control of the impurity concentration is critical.

Heavily $n$ - or $p$-type doped GaAs is used for certain high-speed applications of integrated circuits. An important example is field effect transistors (FETs). Heary doping reduces short channel effects, make FETs more one-dimensional in their electrical performance. and increase their transconductance. High doping of gate regions for FETs reduces leakage currents and increases the available current. Thin base regions in heterojunction bipolar transistors (HBTs). when heavily doped. reduce base resistance and HBT emitters with high concentrations have high switching speeds. GaAs/AlGaAs lasers contain several layers with high doping concentrations for such purposes as blocking currents, producing Ohmic contacts, and changing refractive indices [18]. The above-mentioned devices require carrier concentrations as high as $10^{19} \mathrm{~cm}^{-3}$ in both $\mathrm{n}^{-}$ and $p$-type material. In spite of the big need for high doping concentrations, it has been achieved only using several the state-of-art growth techniques. like metalorganic 
molecular beam epitaxy (MOMBE) [20], molecular beam epitaxy (MBE) [21], and organometallic chemical vapor deposition (OMCVD) [22].

An ideal impurity would possess a high solubility in the host material, a low solid state diffusivity for abrupt dopant profiles, and exhibit little self-compensation. The elements $\mathrm{Be}, \mathrm{C}, \mathrm{Cd}, \mathrm{Mg}$ and $\mathrm{Zn}$ have all been used as acceptor dopants in MOCVD [23]. Among them, $\mathrm{C}, \mathrm{Mg}$, and $\mathrm{Zn}$ are the most commonly used, $\mathrm{Cd}$ and Be less so (in the case of Be, because of its toxicity). There are major advantages of carbon over zinc. Carbon does not have a memory effect. The memory effect stands for a slow approach to steady state after the dopant is initially introduced. Similarly, when the dopant is turned off, the concentration, instead of falling abruptly, decreases slowly [23]. Carbon has a lower diffusion coefficient $[23,29]$ making abrupt carbon doping profiles more easily achieved, and it has higher solubility in GaAs permitting high doping levels [25]. These features are especially desirable for base dopants in devices such as high-gain, high-frequency HBTs. High doping levels allow the formation of non-alloyed Ohmic contacts to the base region of the HBT $[26,27]$. The small diffusivity of the base dopant makes carbon the best choice in HBTs where the base width needs to be narrow [28].

The motivation of this study is to investigate possible chemical changes with respect to carbon in heavily doped GaAs. In this dissertation, I describe results of optical measurements, primarily achieved through Raman spectroscopy, on unannealed and annealed heavily carbon doped GaAs. These measurements provide information mainly on long- and short-range ordering and electronic properties of carbon doped GaAs nondestructively. The most significant outcome of this work is clarifying carbon doping effect on LOPC mode. LOPC mode shift and intensity are investigated for different free 
carrier concentration. Carbon at arsenic site LVM and bandgap are also studied as a function of carrier density. To investigate thermal effects on the crystal quality, impurity incorporation, and doping, some of the samples are annealed. Most of devices undergo thermal cycling during their device fabrication processes. Therefore, it is very important to understand how the electrical and optical properties of the source material change during the heat cycle. Without this information, the exact expectation on the device performance is impossible. Carrier reduction upon annealing is observed by using Raman spectroscopy and photoluminescence. As a possible scenario for carrier density reduction, carbon precipitation is suggested based on Raman spectra taken from annealed samples. 


\section{CHAPTER II}

\section{BACKGROUND}

\subsection{Introduction}

In this chapter, the basic terms used through out this dissertation will be introduced. First of all, local vibration mode (LVM) will be introduced. One of the most drastic changes in vibrational structure due to doping is appearance of LVM. In section 2.2, how the frequency and intensity of LVM are related to chemical impurity incorporation and local ordering will be discussed. Vibrations in crystals are discussed in section 2.3. In GaAs, the lattice vibration is interacting with the vibration of free carriers. The carrier concentration dependence of the LOPC modes in many systems with different plașmon damping constants is studied. As an optical tool in studying these vibrational modes, Raman spectroscopy is used. In section 2.5, basic concepts in Raman spectroscopy are introduced. To get additional information on samples, photoluminescence (PL) is used. Information available through PL measurements is discussed. Annealing effect on material properties is investigated and many studies related to annealing effects on carbon doped GaAs are reviewed.

\subsection{Local Vibrational Modes}

If substitutional impurities present within a semiconductor are lighter than the host atoms, such as $\mathrm{Si}, \mathrm{Be}$ and $\mathrm{C}$ in $\mathrm{GaAs}$, the vibrational amplitude is localized mainly to the light atom and nearest neighbor shell [29]. This is easily understood phenomenologically; the more massive atoms surrounding the lighter impurity cannot 
oscillate at the same high frequency and amplitude. The measurement of such localvibrational modes (LVM), using either Fourier transform infrared (FTIR) or Raman spectroscopy, provides direct information about the lattice location occupied by the light atoms, and even the symmetry and properties of impurities and dopants.

If the impurity vibration is approximated to be completely localized on impurity atom itself, substitutional impurities with a mass $m_{\text {imp }}$ less than that of the host atoms give rise to a LVM with an angular frequency $\omega_{\mathrm{LVM}}$ calculated as follows:

$$
m_{\text {imp }} \ddot{x}=-k_{\text {imp }} x
$$

for harmonic oscillation,

$$
\begin{aligned}
& x=x_{0} e^{i\left(\omega_{I I, I / l}+\phi\right)} \\
& \omega_{I, M M}{ }^{2}=k_{\text {imp }}\left(\frac{1}{m_{\text {imp }}}\right)
\end{aligned}
$$

where $k_{i m p}$ is the force constant between the dopant and its neighbors. LVMs of various impurities in GaAs follow the trend expressed in Eq. 2.2 as shown in Fig. 2.1. However. the delta function model, not surprisingly, is in only rough accord with experiment. It may be improved by taking the vibration amplitude to be non-zero at sites which are nearest neighbors of the impurity as well as the impurity site itself. This is more physical since center of mass of the system must remain fixed. In first-order approximation, we have [30-34]

$$
\omega_{L, M M}{ }^{2}=k_{\text {imp }}\left(\frac{1}{m_{\text {imp }}}+\frac{1}{\chi m_{m m !}}\right)
$$

where $m_{n n}$ is the mass of the nearest neighbors, and $\chi$ is an order-unity parameter that depends on the local angle bending and bond stretching force constants of the particular 


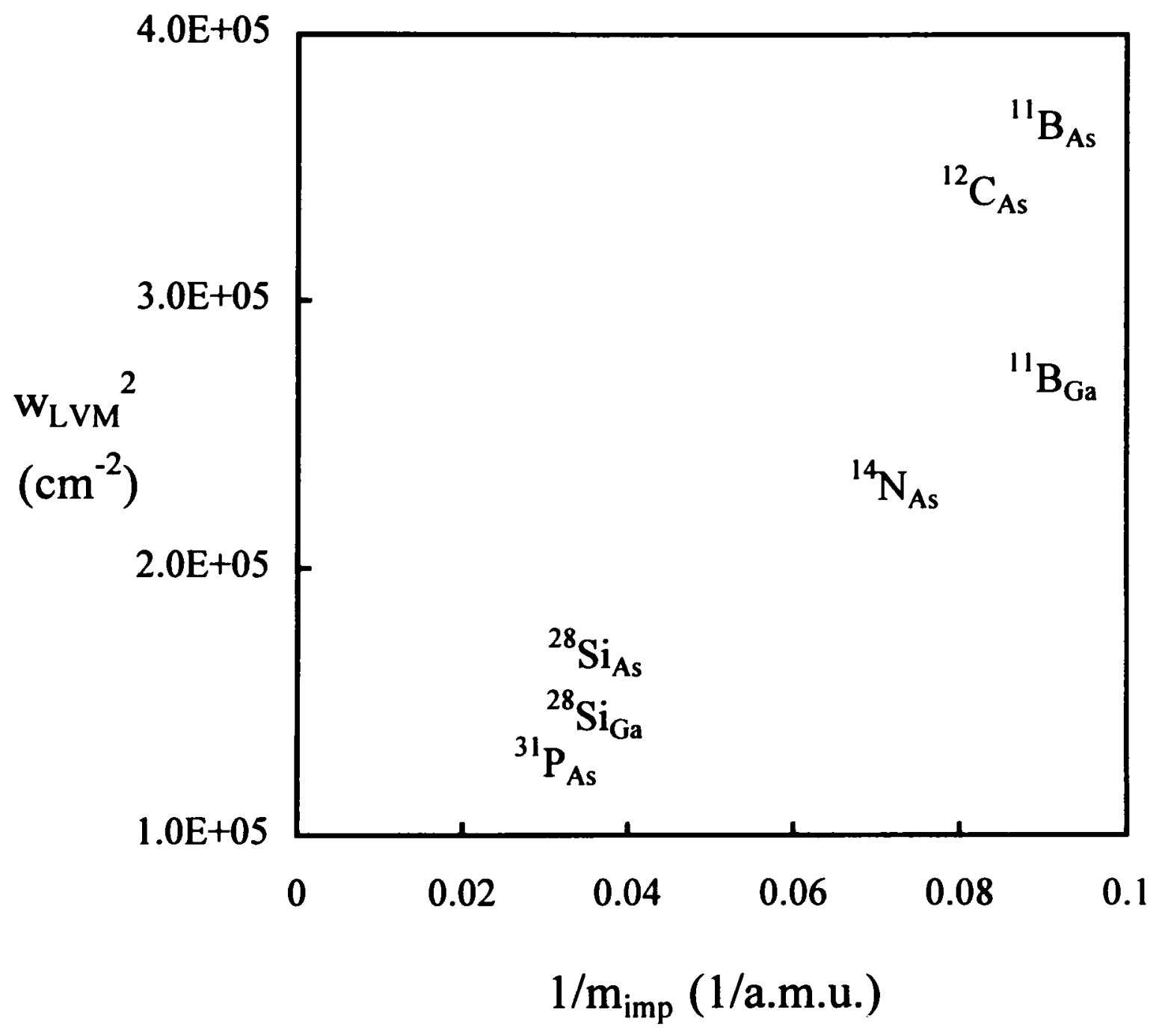

Figure 2.1: Local vibrational mode frequencies in $\mathrm{GaAs}$ due to impurities with different masses. 
vibrational mode. The value of $\chi$ is studied experimentally [35] and theoretically [32]. ${ }^{9} \mathrm{Be}_{\mathrm{Ga}} \mathrm{LVM}$ in GaAs by Wagner et al [36] is observed at $482 \mathrm{~cm}^{-1}$ and ${ }^{28} \mathrm{Si}_{\mathrm{i}, \mathrm{a}} \mathrm{LVM}$ in GaAs by Holtz et al [37] at $381 \mathrm{~cm}^{-1}$. Because the mass of $\mathrm{Si}$ is larger than that of $\mathrm{Be}$. ${ }^{28} \mathrm{Si}_{\mathrm{Ga}}$ LVM has lower frequency than that of ${ }^{9} \mathrm{Be}_{\mathrm{Ga}} \mathrm{LVM}$ according to Eq. 2.3. Furthermore, boron, ${ }^{11} \mathrm{~B}$, LVMs at Ga and As show different Raman shifts, at 517 and $601 \mathrm{~cm}^{-1}$. respectively. because in each case "B has different microstructure. That is. B atoms have different LVM frequencies because they have different neighbors. even though they are the same atoms. Because the mass of As is slightly larger than that of Ga, ${ }^{11} \mathrm{~B}_{\mathrm{Ga}}$ LVM has a lower frequency than that of ${ }^{11} \mathrm{~B}_{\mathrm{A}}$, LVM according to Eq. 2.3. Lightatom site symmetry can be deduced by examining the light polarization properties of the Raman modes. However, substitutional site concentrations are difficult to quantitatively assess using Raman scattering due to the weakness of the LVMs.

GaAs has the zincblende structure as shown in Fig. 2.2. Carbon substitutes for the arsenic atoms in GaAs $\left(\mathrm{C}_{\mathrm{As}}\right)$ making it an acceptor. Like scattering by the LVM produced by $\mathrm{Si}$ on the As site [37, 38], the $\mathrm{C}_{\mathrm{As}}$ LVM Raman line is observed in polarization configurations for which scattering described by a Raman tensor with $\Gamma_{15}$ symmetry is allowed, as shown in Figure 2.3 and Table 2.1. That is, in backscattering along the $z=$ [001] axis, the $C_{A s}$ LVM is allowed in $(x, y)$ and $\left(x^{\prime}, x^{\prime}\right)$, and suppressed in $(x, x)$ and $\left(x^{\prime}, y^{\prime}\right)$ polarization configurations [39]. Here, $x$ and $y$ stand, respectively, for the [100] and [010] crystallographic axes, while $x^{\prime}$ and $y^{\prime}$ are parallel to the [110] and [1 $\left.\overline{1} 0\right]$ directions as shown in Fig. 2.2. Besides the LVM, intrinsic second-order phonon scattering is resolved as a broad band centered at $540 \mathrm{~cm}^{-1}$. The $\mathrm{C}_{\mathrm{As}} \mathrm{LVM}$ is superimposed on the highfrequency edge of these second-order phonon bands. This edge arises from scattering by 


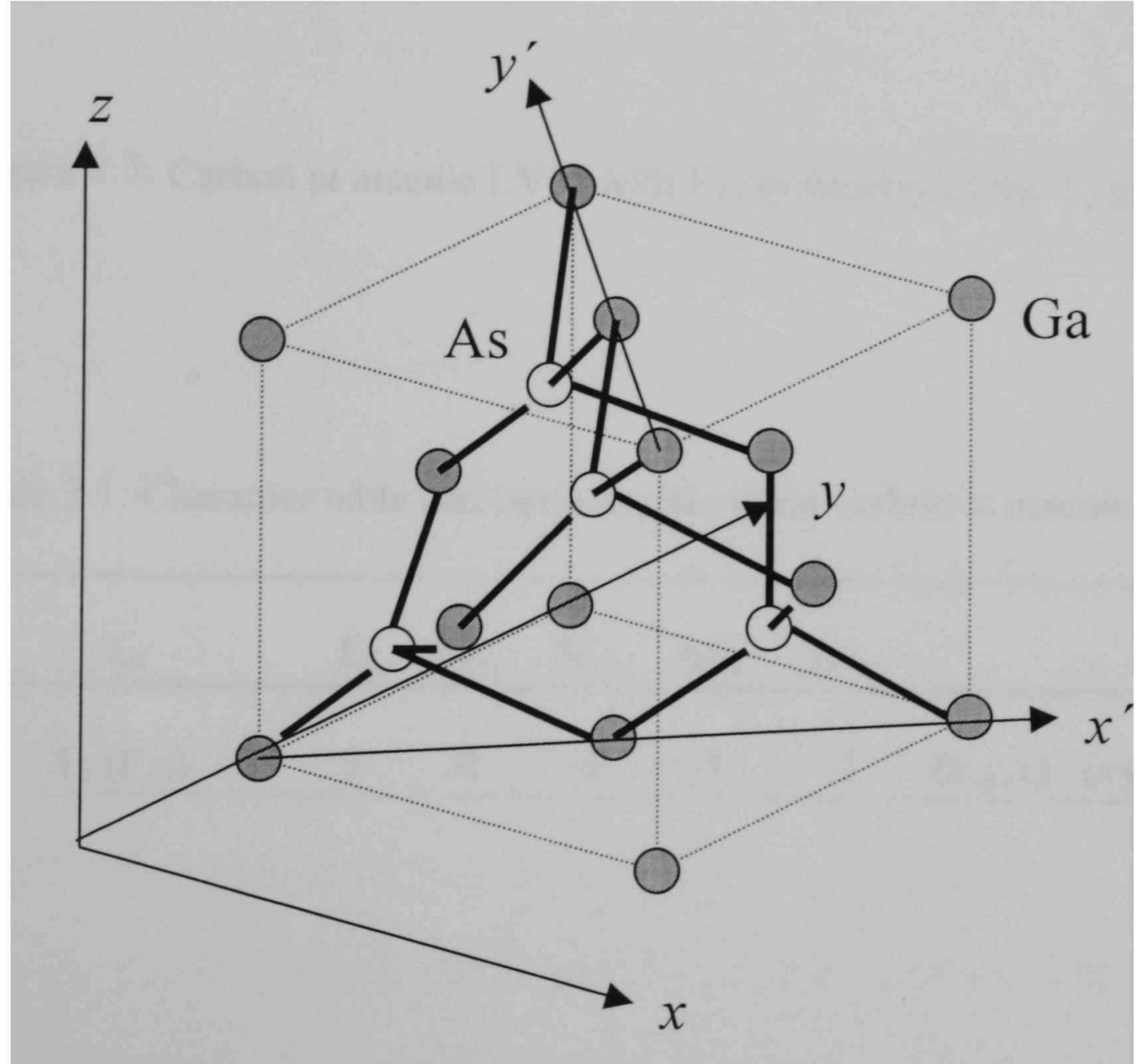

Figure 2.2: Crystal structure of GaAs and its crystallographic axes. 


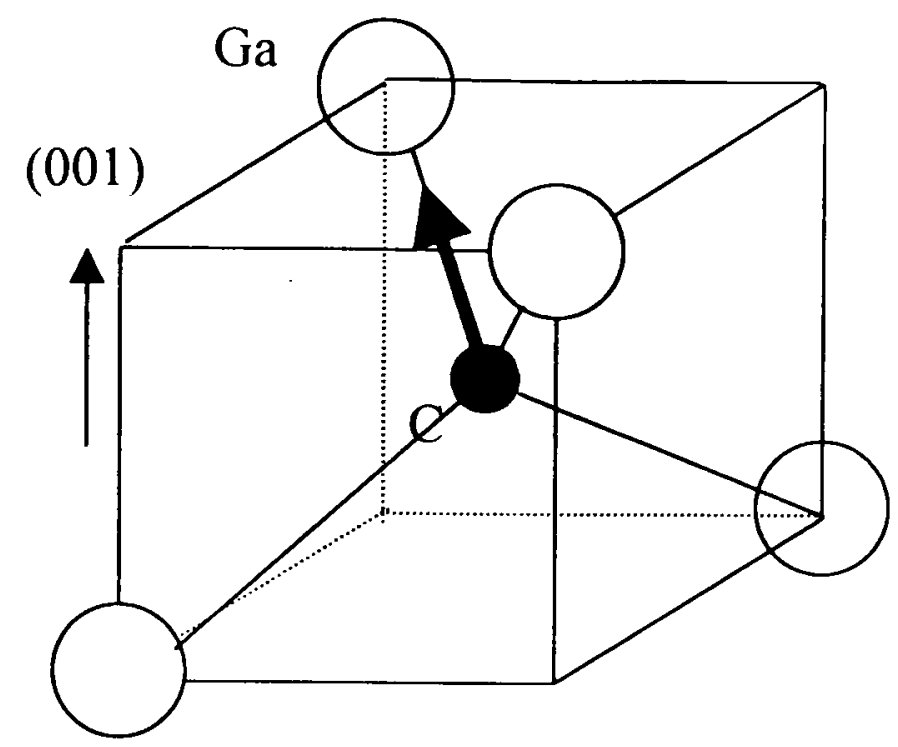

Figure 2.3: Carbon at arsenic LVM with $\Gamma_{15}$ symmetry of the $T_{d}$ group.

Table 2.1: Character table and basis functions for carbon at arsenic LVM.

\begin{tabular}{|c|c|c|c|c|c|c|c|}
\hline $\mathrm{T}_{\mathrm{d}}$ & $\mathrm{E}$ & $8 C_{3}$ & $3 C_{2}$ & $6 S_{4}$ & $6 \sigma_{d}$ & & \\
\hline $\mathrm{T}_{2}\left(\Gamma_{15}\right)$ & 3 & 0 & -1 & -1 & -1 & $(\mathrm{x}, \mathrm{y}, \mathrm{z})$ & $(x y, y z, z x)$ \\
\hline
\end{tabular}


LO phonons at the center of the Brillouin zone [40]. The 2LO( $\Gamma)$-phonon overtone scattering is forbidden in the $\mathrm{z}(\mathrm{x}, \mathrm{y}) \bar{z}$ polarization configuration. Therefore, to study LVM, the $\mathrm{z}(\mathrm{x}, \mathrm{y}) \bar{z}$ polarization configuration is preferred because it allows the LVM while discriminating against $2 \mathrm{LO}(\Gamma)$. The $\mathrm{C}_{\mathrm{As}} \mathrm{LVM}$ peak is identified by Wagner et al. [39] at $582 \mathrm{~cm}^{-1}$.

The intensity of LVM has been correlated with the dopant concentration [31]. The intensity $\mathrm{I}_{\mathrm{LVM}}$ is expected to increase with increasing dopant concentration from the relation $[36,39]$

$$
\mathrm{I}_{\mathrm{LVM}} \sim[\text { dopant concentration }] \times \mathrm{V} \times \sigma,
$$

where $\mathrm{V}$ denotes the scattering volume, and $\sigma$ the scattering cross section per impurity For $\sigma$ independent of the doping level a proportionality of the LVM intensity to the dopant concentration is obtained under constant scattering volume conditions.

For C-doped GaAs studied by Wagner et al. [39] their experimental data are described by the relation, $\mathrm{I}_{\mathrm{LVM}} \sim[\mathrm{C}]^{2 / 3}$. This exponent is unexpected. As discussed by Wagner et al., the proportionality accounted for all carbon, not only that which is substituted onto arsenic sites. The conclusion by Wagner et al. that $\mathrm{I}_{\mathrm{LVM}} \sim[\mathrm{C}]^{2 / 3}$ raises an interesting question: Where does the additional carbon reside in the GaAs crystal? This is discussed in Ch. $\mathrm{V}$ of my dissertation. As an alternative explanation for $\mathrm{I}_{\mathrm{LVM}} \sim[\mathrm{C}]^{2 / 3}$, Wagner et al. suggested a concentration-dependent scattering cross section as observed for B in Si system. [41] This is explained by an additional scattering mechanism due to the Coulomb field associated with the B atom. This field is screened by free carriers at high doping level. Due to the short history of GaAs:C, and the weakness of the $\mathrm{C}_{A_{S}}$ LVM, there are few Raman studies on this mode. 
The LVM of Be in GaAs was studied by Wagner et al. [36]. The observed proportionality between normalized LVM intensity with respect to the intrinsic secondorder phonon scattering at $540 \mathrm{~cm}^{-1}$ and $\mathrm{Be}_{(\mathrm{ia})}$ that is. $\mathrm{I}\left(\mathrm{Be}_{(\mathrm{ia})}\right)^{\prime} \mathrm{I}\left(5+0 \mathrm{~cm}^{-1}\right)=\left[\mathrm{Be}_{(\mathrm{ia}}\right]$. indicates a concentration independent scattering cross section. Thus, the second-order phonon structure serves as an internal intensity standard that is proportional to the scattering volume in Eq. 2.4. This proportionality can be used to nondestructivel! determine the carrier concentration of a sample. once a calibration between LVM intensity and carrier density has been established.

\subsection{Vibrations in Crystals}

Every atom inside a crystal is vibrating. The motion of each atom is affected by its neighbors and therefore all crystal oscillations are coupled together. The coupling can be strong. as in lattice vibrations, or weak, as in local modes. Motion of this type can be quite complex if it is described in ordinary coordinates that describe the geometrical configuration of the system. The coupled motion can be described in a simpler way by introducing normal coordinates that are constructed from the original position coordinates in such a way that there is no coupling among the oscillators. Thus each normal coordinate oscillates with a single. well-defined frequency [ [2].

\subsubsection{Linear Diatomic Chain}

Normal modes in one-dimensional diatomic chain system. $\mathrm{N}$ of each atom. can be calculated as follows. based on Ashcroft and Mermin [43] primary reference. If we 
assume each atom interacts only with its nearest neighbor with spring constant $\mathrm{C}$ and $\mathrm{a}$ is the repeat distance of the unit cell, then the equations of motion are

$$
\begin{aligned}
& M \dot{x}_{1 ! n}(t)=C\left(x_{m m}+x_{m m-1}-2 x_{1 m}\right) \\
& m \ddot{x}_{m ! n}(t)=C^{\prime}\left(x_{1 ! n}+x_{1 m+1}-2 x_{m m}\right)
\end{aligned}
$$

where $x_{1 / n}$ and $x_{m n}$ are displacements of $\mathrm{M}$ and $\mathrm{m}$, respectively. in the nth primitive cell. We look for a solution in the form of a traveling wave. with different amplitudes $A_{1}$ and $\mathrm{A}_{\mathrm{m}}$

$$
\begin{aligned}
& x_{1 / n}(t)=A_{11} e^{(k m m-c(x)} \\
& x_{m ! n}(t)=A_{m} e^{\left(1 k\left(n+\frac{1}{2}\right)(u-t(x)\right.}
\end{aligned}
$$

where $\mathrm{k}$ is the wavevector and $\omega$ is the frequency. From the Born-von Karman periodic boundary condition, i.e.. $x_{1 / n+.}=x_{1 / n}$, we have the following conditions.

$$
k=\frac{2 \pi h}{N a} . \text { where }-\frac{N}{2} \leq h \leq \frac{N}{2}
$$

On substitution of Eq. 2.6 into Eq. 2.5 we obtain

$$
\begin{aligned}
& -M \omega^{2} A_{M}=2 C\left[A_{m \prime} \cos \frac{k a}{2}-A_{1 \prime}\right] \\
& -m \omega^{2} A_{m}=2 C\left[A_{11} \cos \frac{k a}{2}-A_{m}\right] .
\end{aligned}
$$

These homogeneous linear equations have a non-trivial solution only if the determinant of the coefficient of $A_{11}$ and $A_{m}$ vanishes

$$
\left|\begin{array}{lc}
2 C-M \omega^{2} & -2 C \cos \frac{k a}{2} \\
-2 C \cos \frac{k a}{2} & 2 C^{\prime}-m \omega^{2}
\end{array}\right|=0 .
$$

The solutions of the above equation are 


$$
\omega^{2}=C \frac{M+m}{M m} \pm \sqrt{\left(\frac{M+m}{M / m}\right)^{2}-\frac{t}{1 / m} \sin ^{2} \frac{k a}{2}} .
$$

There are $2 \mathrm{~N}$ normal modes. For each $\mathrm{k}$, there are 2 values of $\omega$. The lower branch of $\omega$ is called the acoustic branch. and the upper one is the optical phonon branch. The dispersion curve from Eq. 2.10 is shown in Fig. 2.4 (a). At the zone center like points A and D in Fig. 2.4. both of the atoms move. At point A. atoms move in opposite directions and center of mass is stationary. At point B, atoms move in the same direction with nearly the same amplitude. The motion is $180^{\circ}$ out of phase in the neighboring unit cell. Only light (heavy) atoms move at the zone edge point B (C). Not all the normal mode is detected by Raman spectroscopy. Even though DOS of point A. B. and C are all large. only the mode of point A shows up clearly because of $\vec{k}=0$ selection rule. This selection rule will be derived and described in more detail in section 2.5.

\subsubsection{Vibrational structure of GaAs}

In a diatomic three-dimensional system such as is relevant for zincblende crystals. there are $3 \times(2 \mathrm{~N})=6 \mathrm{~N}$ normal modes. Of these $3 \mathrm{~N}$ make the three acoustic modes. and the other $3 \mathrm{~N}$ correspond to the three optic modes. For $\vec{k}$ along a symmetry axis of the solid, two modes of each acoustic and optic mode are transverse, and the other is longitudinal. The two transverse modes are degenerate along the symmetry axes. The dispersion curve for GaAs [44] is shown in Fig. 2.4 (c) as an example of diatomic three dimensional system. The dispersion curve of pure GaAs shows the characteristics mentioned above. However in doped GaAs, the LO phonon energy is less that that of pure GaAs LO phonon mode. This was suggested by Cowley et al. [45] as due to plasma 


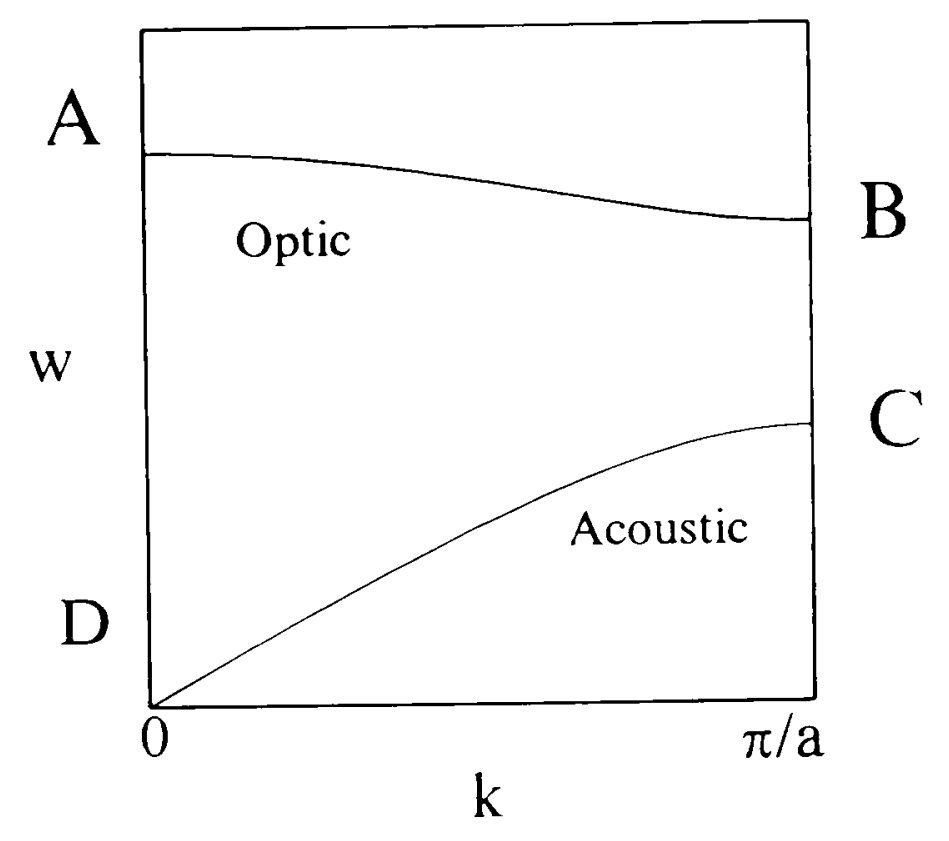

(a)

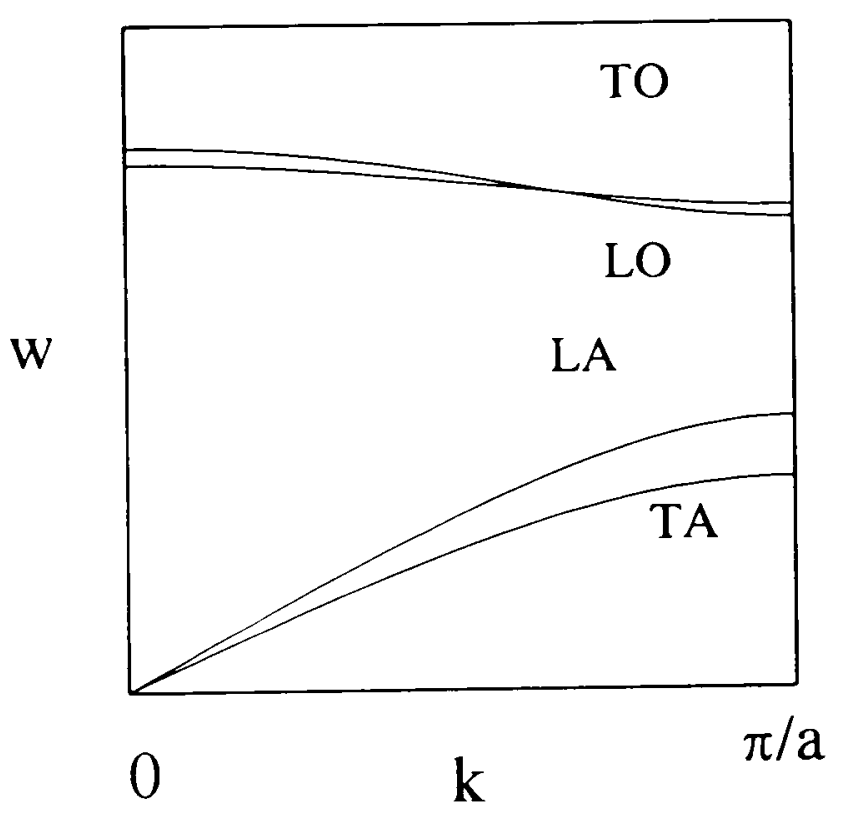

(b)

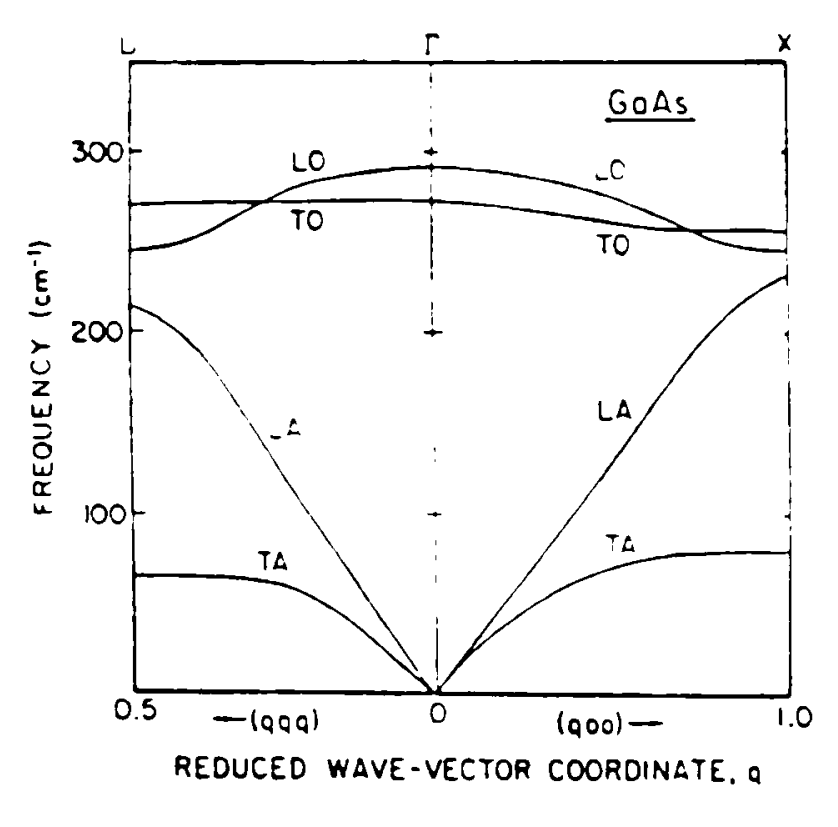

(c)

Figure 2.4: Dispersion curves of (a) 1-Dim. diatomic. (d) 3-Dim. diatomic systems and (c) GaAs [136]. 
screening of the macroscopic electric field produced by LO phonon based on the following relation [46]:

$$
\omega_{1,1}^{2}=\omega_{11}^{2}+C E_{11}
$$

where $\omega_{\mathrm{LO}}\left(\omega_{\mathrm{TO}}\right)$ is the frequency of the $\mathrm{LO}(\mathrm{TO})$ phonon mode, and $\mathrm{E}_{11}$ is a macroscopic electric field. $\mathrm{C}$ is a constant depending on the masses and effective charges of the ions.

\subsection{Longitudinal optic- plasmon coupled modes}

In GaAs, an oscillating electric dipole moment is generated by the LO phonons due to the lack of inversion symmetry. The electric dipole moment interacts with the longitudinal plasma oscillations of the free carriers. The LOPC mode frequencies can be theoretically deduced by solving the set of equations established for the coupled system. In this section, the interaction between lattice vibration and free carrier plasmons will be discussed, and expressions used to calculate the interaction will be described.

\subsubsection{Low damping and $\vec{k}=0$ case}

In the long wavelength limit. the optical ribrations of a diatomic lattice correspond to the motion of one type of atom are all in phase, relative to the other kind. In GaAs, such motion is associated with oscillating electric dipole moments. If $x$ represents the displacement of the positive ions relative to the negative ions. a reduced displacement vector $u$ may be expressed as $u=x\left(\mu / V_{s}\right)^{1 / 2}$, where $\mu / V_{s}$ is the reduced mass per Bravais unit cell. Then. the macroscopic equations describing the polar motion are 


$$
\begin{aligned}
& \ddot{\vec{u}}=k \vec{u}+e_{1} \vec{E} \\
& \vec{P}=e_{r} \vec{u}+\chi_{i} \vec{E} .
\end{aligned}
$$

From the above equations, anharmonicity and higher-order terms in the electric moment are neglected and $k, e_{T}$, and $\chi_{i}$ are the force constant, Born`s transverse effective dynamic charge, and polarizability without lattice vibration. respectively. $\vec{E}$ and $\vec{P}$ are the electric field and the dielectric polarization. The dielectric polarization $\vec{P}$ and the dielectric function $\varepsilon$ are defined as follows.

$$
\begin{gathered}
\vec{P}=\chi \vec{E} \\
\varepsilon=1+4 \pi \chi
\end{gathered}
$$

where $\chi$ is the polarizability. By using the periodic solutions.

$$
(\vec{u}, \vec{E} \cdot \vec{P})=\left(\vec{u}_{0}, \vec{E}_{0}, \vec{P}_{0}\right) e^{\imath(u t \prime}
$$

the polarization and dielectric function are represented as:

$$
\begin{gathered}
\vec{P}=\left(\chi_{1}+\frac{e_{1}{ }^{2}}{-k-\omega^{2}}\right) \vec{E} \\
\varepsilon=1+4 \pi \chi_{1}+\frac{4 \pi e^{2}}{-k-\omega^{2}} .
\end{gathered}
$$

From the similarity of Eq. 2.17 to the infrared dispersion formula,

$$
\varepsilon=\varepsilon_{0}+\frac{\left(\varepsilon_{0}-\varepsilon_{s}\right) \omega_{0}^{2}}{\omega_{0}^{2}-\omega^{2}}
$$

the parameters $k, e_{T}$, and $\chi_{i}$ are obtained. In the above equation, $\omega_{0} . \varepsilon_{0}\left(\varepsilon_{x}\right)$ are the dispersion frequency and low (high) frequency dielectric constant. 


$$
\begin{aligned}
& k=-(1)_{11}^{2} \\
& \mathfrak{e}_{1}=\omega_{11} \sqrt{\frac{\varepsilon_{01}-\varepsilon_{x}}{4 \pi}} \\
& \chi_{1}=\frac{\varepsilon_{x}-1}{4 \pi} \\
& \omega_{0}=\omega_{l()} \\
& \omega_{l, 0}=\sqrt{\frac{\varepsilon_{0}}{\varepsilon_{x}} \omega_{l()}}
\end{aligned}
$$

Therefore. the dielectric function of the system under only polar lattice vibration is

$$
\varepsilon=\varepsilon_{0}+\frac{\varepsilon_{x}\left(\omega_{l, .}^{2}-\omega_{\%,}^{2}\right)}{\omega_{\%,}^{2}-\omega^{2}-i \gamma \omega} .
$$

The above equation is generalized to include the phonon damping effects. with phonon damping constant $\gamma$.

In order to account for free carrier effects, the motion of free carrier in terms of its effective mass $\mathrm{m}^{*}$ and scattering time $\tau$ is considered.

$$
m^{*} \ddot{\vec{X}}+\frac{m^{*}}{\tau} \dot{\vec{X}}=e \vec{E} e^{\prime \prime \omega t}
$$

Then the polarizability. $\vec{P}_{f}$, associated with the free carriers can be written down as

$$
\vec{P}_{f}=n e \vec{X}=\frac{n e^{2} / m^{*}}{-\omega^{2}-i \omega \Gamma} \vec{E}
$$

where $\mathrm{n}$ is the number of free carriers per unit volume, and $\Gamma$ is the plasmon damping constant defined by the inverse of $\tau$. Therefore. the dielectric function of the system under phonon and plasmon interaction can be calculated as follows:

$$
\varepsilon(\omega)=\varepsilon_{\infty}+\frac{\varepsilon_{\infty}\left(\omega_{l .,}^{2}-\omega_{T()^{2}}{ }^{2}\right)}{\omega_{T(}{ }^{2}-\omega^{2}-i \omega \gamma}-\frac{\omega_{p}{ }^{2} \varepsilon_{x}}{\omega(\omega+i \Gamma)}
$$


where $(1)_{p}$ is the plasma frequency defined as

$$
\omega_{p}^{2}=\frac{4 \pi n n^{2}}{\varepsilon, m^{-}}
$$

The coupled plasmon-LO phonon mode frequencies are calculated from the routs if :.(1.) as follows.

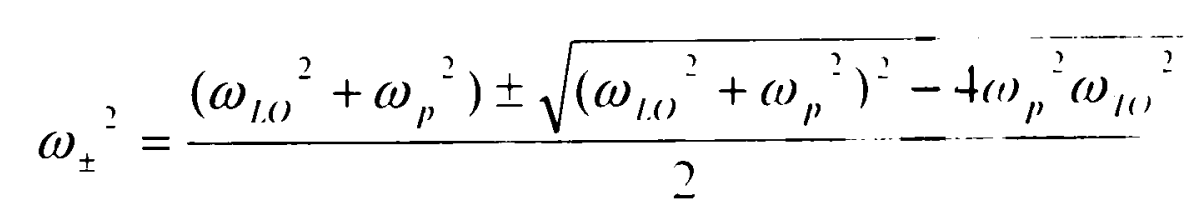

In the above equation, phonon and plasmon damping are neglected for their smallness, and $\omega_{ \pm}$represent frequencies of two LOPC modes, denoted $\mathrm{L}_{ \pm}$, respectively. Because $\omega_{\mathrm{p}}$ is related to carrier concentration, $\omega_{ \pm}$can be expressed as a function of $n$, and plotted as shown Fig. 2.5 [44]. The L. mode has an energy which varies between zero and that of the transverse-optic (TO) phonon energy in the long-wavelength limit, depending on the carrier concentration. The $\mathrm{L}_{+}$band is very close to the $\mathrm{LO}$ phonon at low $n$ and increases in energy as the carrier concentration increases. This behavior was observed in $n$-type GaAs by Mooradian and Wright [47]. In their $n$-type GaAs system, relatively free electrons in the conduction band are the participants in plasma-like oscillations. To good approximation, the single effective mass of the conduction-band electrons describes the plasma. These modes serve as a good measure of carrier concentration [47].

2.4.2. Low damping and $\vec{k} \neq 0$

LOPC modes with $\vec{k} \neq 0$ scattering wavevector were studied in n-typc (iills h using various excitation lines [48, 49]. From these studies, the LoP(' modes with $\vec{k} \leq 10^{-2} \times k_{\max }$ are studied. The wave vector non-conservation was associated with the 


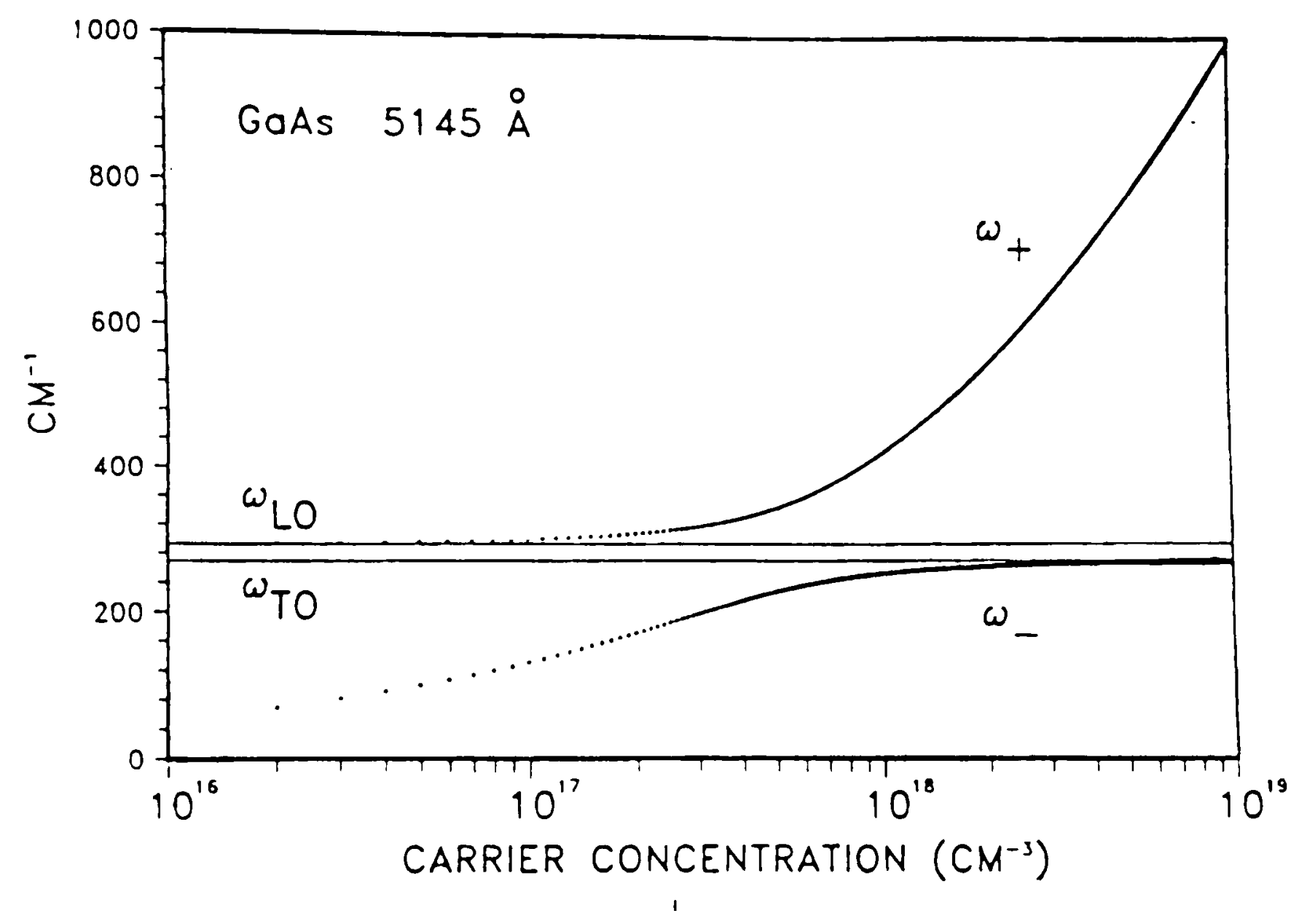

Figure 2.5: Calculated plasmon-phonon mode frequencies as a function of free carrier concentration for Raman scattering from GaAs at $514.5 \mathrm{~nm}$. The solid lines correspond to the bulk LO and TO modes. The calculations have been corrected for finite k-rector and non-parabolic effective mass effects [136]. 
absorption of incident and scattered light in an opaque crystal [49]. The dispersion curves for LOPC bands are plotted for different carrier concentrations. For small waverectors. $\mathrm{L}_{+}$mode is stationary as a function of scattering waverectors and the coupled modes are sharp. As the scattering wavevector increases. L. and $\mathrm{L}_{+}$modes are blue shifted for a given carrier concentration. L- mode shows a downward bending at $\vec{k} \approx 1.0 \times 10^{6} \mathrm{~cm}^{-1} \approx 10^{-2} \times k_{\max }$ as shown in Fig. 2.6. This bending is analyzed by Abstreiter et al. [49] as due to the strong absorption.

\subsubsection{High damping}

In p-type GaAs, the high-energy plasmon-like LOPC mode is weak. resulting in a single mode near the LO and TO phonons [50]. Recently, this behavior has been explained by using higher hole effective masses, lower hole mobility, and higher plasmon damping constant $[51,52]$. These effects weaken and broaden the high-energy plasmonlike LOPC mode $[51,52]$. To date this mode has not been convincingly detected in $p$ type GaAs. Therefore. single-mode LOPC behavior is seen in $p$-type material when low carrier mobility. high damping constant, and higher effective carrier mass are characteristic for the plasma oscillations. Similarly, a single LOPC band is observed in $n$ type samples where the same characteristics apply [53-58].

The LOPC mode in p-type GaAs is a complicated phenomenon. Holes near the valence band edge carry the amplitude of the plasma oscillations. However, the structure of the valence band edge of GaAs is rich. exhibiting heavy and light hole states. with their respective effective masses. and the nearby split-off hole band. Even though only a fraction of carriers are light holes [59], 


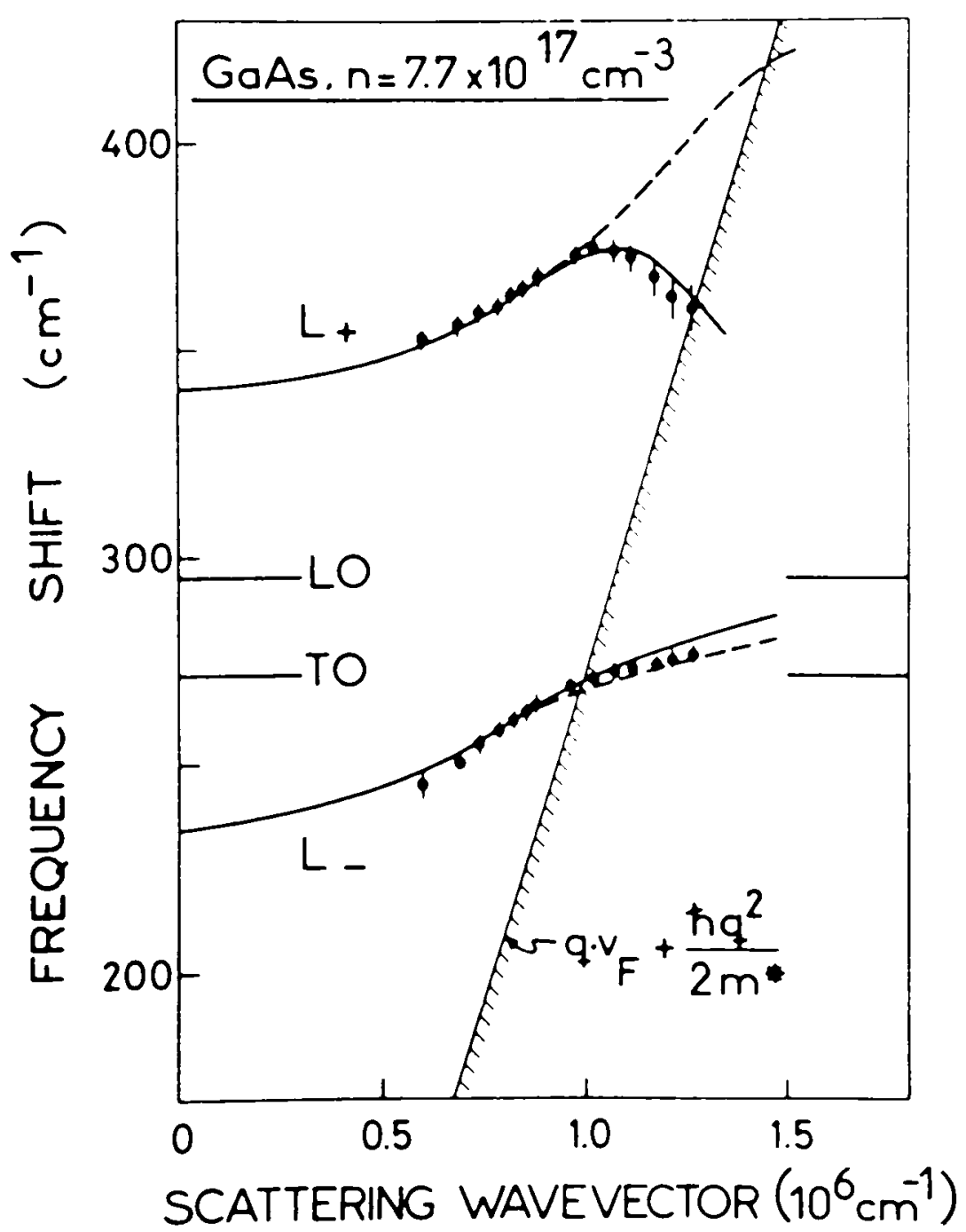

Figure 2.6: Dispersion of the peaks in the Raman spectra of coupled plasmon-LO phonon modes of $n$-GaAs. The solid and dashed lines are calculated by Abstreiter et $a l .[+9]$ the dots represent the experimental results. 


$$
\frac{N_{l l t}}{N_{l \prime}}=\left(\frac{m^{*}{ }_{l \prime \prime}}{m^{*}{ }_{l \prime \prime}}\right)^{3 / 2} \approx 17
$$

their influence on the optical and electrical properties of the plasma cannot be ignored due to their comparatively large plasmon energy and higher mobility. This necessitates the use of at least two hole masses in describing the plasma [51]. The electronic susceptibility must account for light and heavy hole state intraband transition contributions $[52-54,57,58,60-64]$. Recent work has included contributions to the susceptibility due to transitions between these valence bands $[51,59,65]$. Another factor is breakdown of the wave vector $\vec{k}=0$ selection rule due to the strong scattering of holes by ionized impurities in the Raman process. This effect is of greatest relevance for extremely high dopant-ion concentrations [66]. Two schemes are used to explain the LOPC mode in $p$-type GaAs. The first involves large wave vector modes due to the selection rule breakdown described above [66, 67]. The second scheme considers strong damping in the long wavelength limit [59.62]. We adopt here the latter approach which is found to adequately describe the results of Be doped GaAs [59.62].

The simplest type of light scattering related to the phonon component of the coupled mode is the deformation potential mechanism [68]. A long wavelength optical phonon involves relative displacements of atoms within the primitive cell by changing bond lengths and/or the bond angles, causing microscopic distortions. In this mechanism the lattice vibrations modulate the electron energy band structure and thereby cause a modulation in the electric susceptibility related to interband transitions. Therefore. the deformation potential is the parameter that describes the change of energies at different 
Brillouin zone introduced by the distortions. An additional contribution arises from the macroscopic electric field that accompanies the coupled modes. This field also produces a modulation in the interband electric susceptibility. W'ithin perturbation theory these effects are described as field-induced intraband and interband transitions. This description leads to two-band and three-band representations of the light scattering cross section [69]. The three-band electric field contributions are referred to as the electro-optic mechanism, and two-band electric field contributions as the Fröhlich mechanism. In polar semiconductors like GaAs, a long wavelength LO phonon involves uniform displacements of the charged atoms within the primitive cell. Such relative displacement of oppositely charged atoms generates a macroscopic electric field. This electric field can interact with free carriers. This electron LO phonon interaction is known as the Fröhlich interaction. The electronic transitions involved in the calculation of the transition susceptibility may be described by diagrams of the type shown in Figure 2.7 (a) and (b) [69]. These two interactions are used to explain the observed LOPC behavior in $\mathrm{Zn}$ doped GaAs [66]. Be doped GaAs [52], and Ge doped GaAs [67]. In the case of crystals having free carriers, it is also possible to have initial or final electronic transitions which are intraband. This is shown in the diagrams of Fig. 2.7 (c) for the case of a degenerate $n$ type semiconductor. Consequently, crystals with free carriers have contributions to the transition susceptibility which do not exist for crystals with full valence bands and empty conduction bands. These additional contributions. which are associated with the charge density fluctuations of the electron-gas. are important light scattering mechanisms for semiconductor plasmons [69]. 

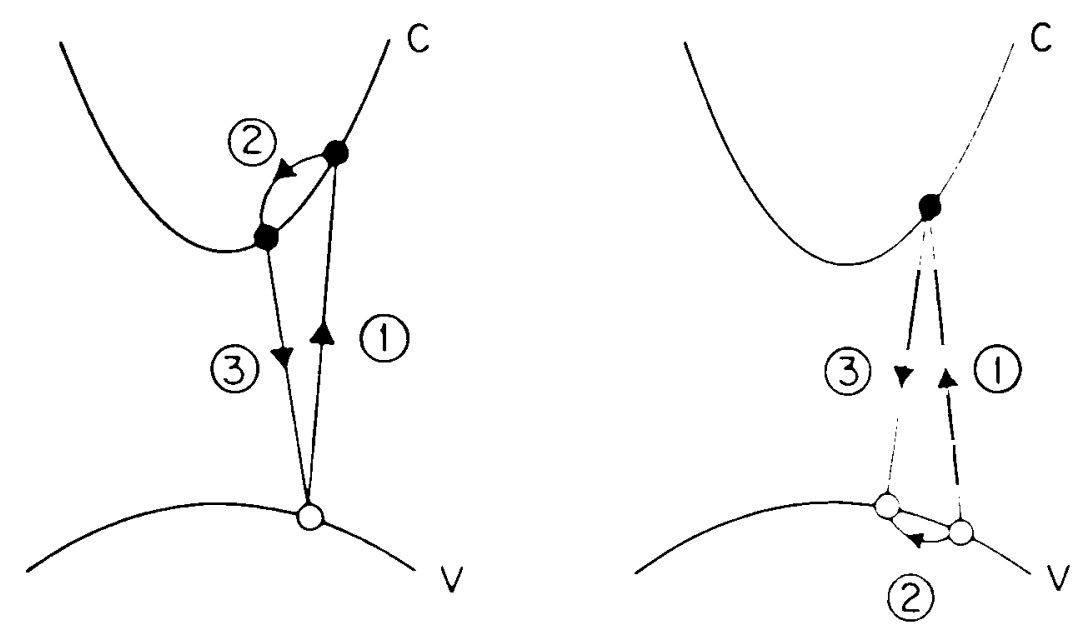

(a)

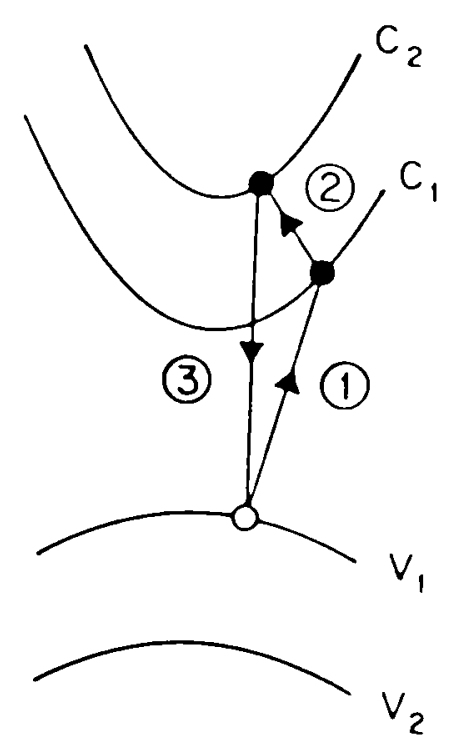

(b)
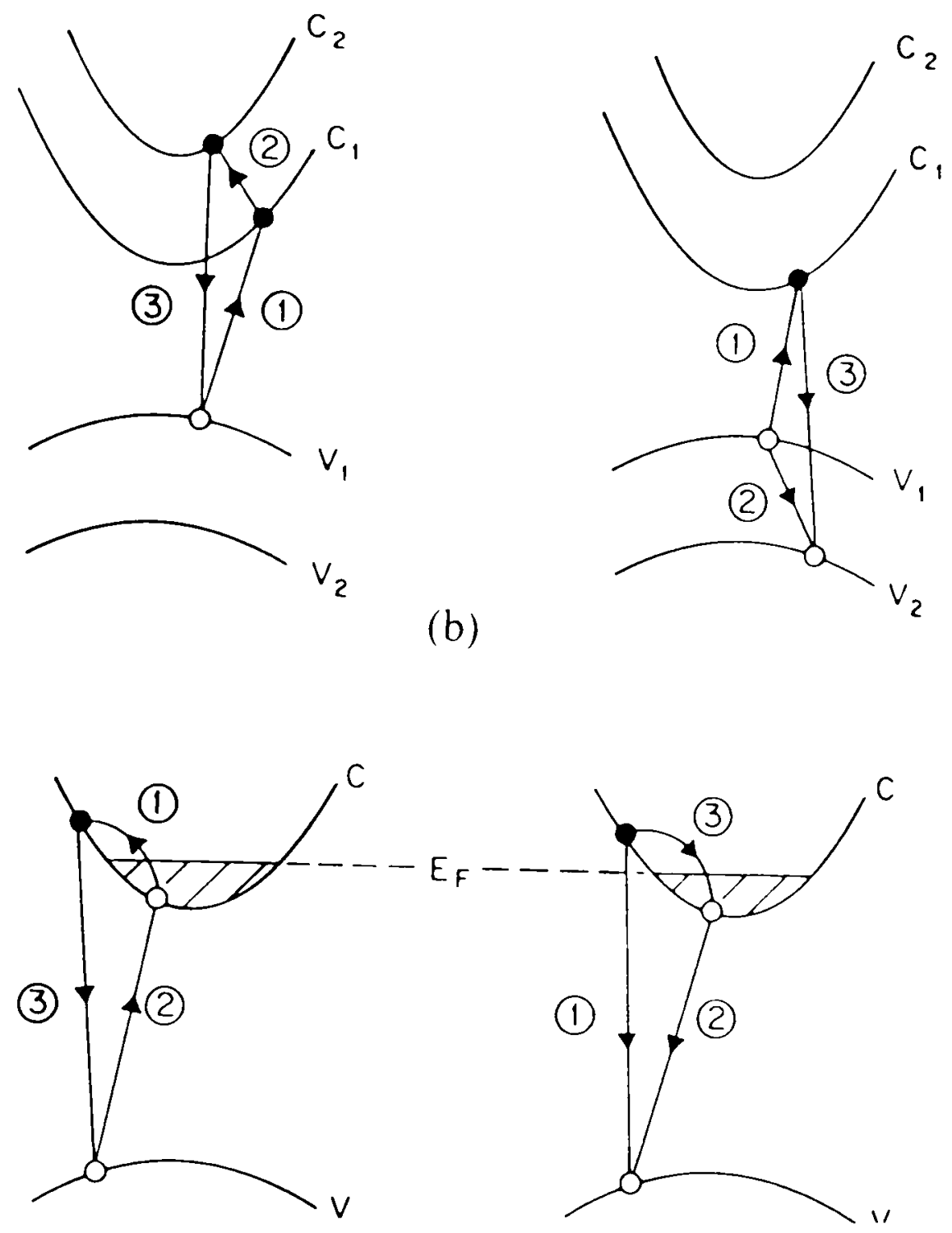

(c)

Figure 2.7: Schematic diagrams of the interband and intraband electronic transitions which play a role in (a) two-band and (b) three-band Raman processes. (c) Schematic diagrams of the additional interband and intraband electronic transitions which playa role in scattering processes which take place in an $n$-type semiconductors [69]. The numbers indicate the order of the electronic transitions. $\mathrm{C}, \mathrm{C}_{1}$ and $\mathrm{C}_{2}\left(\mathrm{~V}, \mathrm{~V}_{1}\right.$, and $\left.\mathrm{V}_{2}\right)$ are conduction (valence) bands. 


\subsection{Raman Spectroscopy}

When monochromatic radiation of warenumber $\overline{l^{\prime}} \equiv 1 / \lambda$ is incident on systems like transparent solids. some scattering of radiation occurs. The scattered radiation is composed of wavenumber $\bar{v}$ associated with the incident radiation, and new wavenumbers of $\bar{v} \pm \Delta \bar{v}$. The wavenumber difference between the incident and scattered light $\Delta \bar{v}$ are related to transitions between rotational, vibrational, and electronic levels. The scattered radiation has polarization characteristics which can be different from those of the incident radiation. Both the intensity and the polarization of the scattered radiation depend on the direction of observation [70].

Such scattering of radiation with change of wavenumber is first observed by C.V. Raman [71] in 1928. The new wavenumbers are termed Raman lines. or bands, and collectively said to constitute a Raman spectrum. Raman scattering occurs by phonon annihilation (Stokes bands with wavenumbers $\bar{v}-\Delta \bar{v}$ ). and phonon creation (antiStokes bands with wavenumbers $\bar{v}+\Delta \bar{v}$ ). It is a little difficult to make generalization about the relative intensities of Stokes and anti-Stokes bands. Looking at the thermal dependence only, the ratio of Stokes to anti-Stokes is

$$
\frac{I_{S}}{I_{A S}}=\frac{n_{B}(h \Delta \bar{v} \cdot T)+1}{n_{B}(h \Delta \bar{v} \cdot T)}=e^{\frac{h S_{B}}{\hat{k}_{k} T}}
$$

where Bose function is used because phonons are bosons. The intensity of anti-Stokes is weaker than that of Stokes Raman scattering. and the ratio between these two decreases rapidly with increase in the wavenumber shift as shown in Eq. 2.28 . 
As mentioned before, the change of wavenumbers in scattered light. $J \bar{v}$. is related to rotational, vibrational. and electronic transitions. Usually, rotational transition energy is in the range of $10^{-4} \mathrm{eV}$. vibrational transition in $10^{-2} \mathrm{eV}$, and electronic transition is related to much larger energy as shown in Fig. 2.8. Therefore. Raman spectra may be used to study rotational and vibrational characteristics of the material. For gas system, the Raman spectrum will consist of a series of rather finely spaced lines of relatively small wavenumber shift. arising from transitions between the closely spaced rotational levels and a number of other lines of larger wavenumber shift, arising from transitions which involve either a change of vibrational state or a simultaneous change of vibrational and rotational states. For liquid and solid samples, the Raman spectrum will usually show only Raman lines arising from vibrational transitions since free rotation is inhibited.

Not every vibrational transition is Raman active. The first selection rule is related to polarizability tensor. In order for a vibration to be Raman-active. the change in polarizability of the molecule with respect to vibrational motion must not be zero at the equilibrium position of the normal vibration, i.e..

$$
\left(\frac{\partial \alpha}{\partial r}\right)_{r} \neq 0
$$

where $\alpha$ is the polarizability and $r_{\mathrm{e}}$ represents the equilibrium distance along the normal coordinate. In general. for any molecule that possesses a center of symmetry, there will be no fundamental lines in common in the infrared and Raman spectra. This is a very valuable generalization for structure determination. 


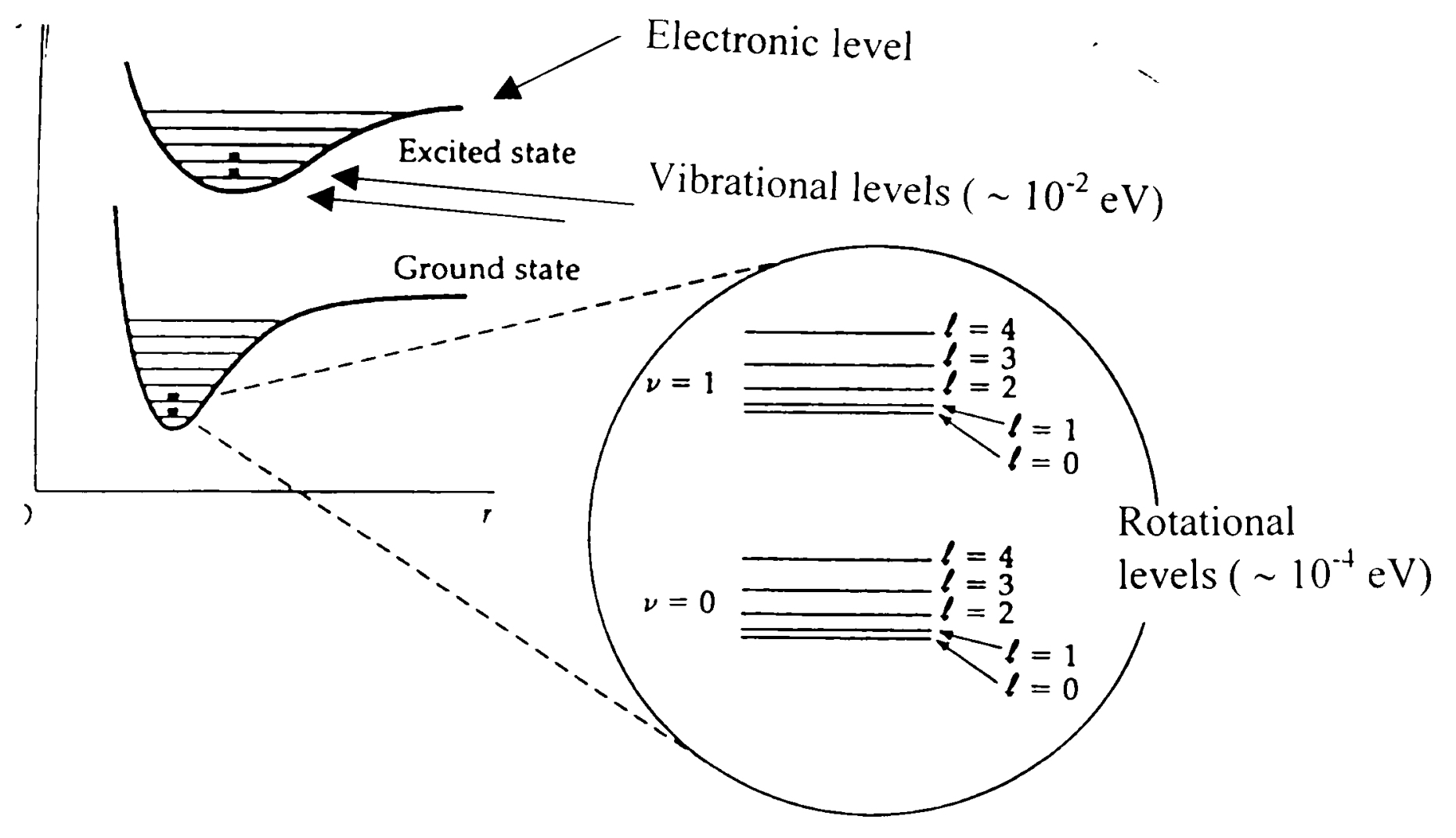

Figure 2.8: Energy level diagram for rotational, vibrational and electrical levels. 
The second selection rule can be derived from the harmonic oscillator approximation. This selection rule. which is rigorous for a harmonic oscillator. states that in the absorption of radiation only transitions for which change of vibrational quantum number $= \pm 1$ can occur. Therefore. only Raman lines $w$ ith fundamental frequency are detected from harmonic oscillator approximation. Since most molecules are not perfect harmonic oscillators, this selection rule breaks down and lines corresponding to overtones are detected.

Raman is an extremely useful method for the characterization of semiconductors and is an important complement to other spectroscopic techniques because it is a function of electron-phonon (lattice vibration) interactions. Raman spectroscopy is a very useful method to study lattice vibrations and their interactions with other excitations such as plasmon oscillations. Since lattice vibrations are very sensitive to local environment. Raman scattering can give information about material structure and quality on the scale of a few lattice constants. Raman spectroscopy is an effective method for studying both local structure and long-range crystalline order, plus the effects of doping [4+]. Also. since Raman scattering is a second-order optical process. it contains important symmetry information not available from first-order interaction. For example, in Raman scattering the scattered light intensity for a given phonon mode is a function of both the incident and scattered light polarizations. Therefore, polarization study reveals the symmetry information that can be used to characterize many material parameters. In Raman scattering peak positions as well as polarization selection rules are sensitive to perturbations. like electric field. strain. pressure. and temperature. Furthermore by using 
various excitation lines with different wavelength it is possible to perform nondestructive depth profiling measurements and excite resonance interactions because the absorption coefficient varies with $\lambda$.

In a Raman experiment, a monochromatic beam of light illuminates the sample. In transparent media it is possible to observe the scattered light at right angles to the incident beam $[44,72]$. However, if the crystal is not transparent to the incident radiation, as is the case for most semiconductors, the observation is done in the back scattering geometry. The polarizability tensor $\chi$ defined by Eq. 2.13 can be expanded as follows.

$$
\chi_{i m}=\chi_{i m}{ }^{0}+\chi_{i m}{ }^{1} u+\chi_{i m}{ }^{2} u^{2}
$$

where $u$ is a nuclear displacement during a normal vibration. Thus, for the $j$ th normal mode

$$
u_{i}=u_{j 0} e^{i \omega_{i}^{\prime}}
$$

and the electric field of frequency $\omega_{i}$

$$
\vec{E}=\vec{E}_{0} e^{i \omega, t}
$$

the polarization can be expressed as following.

$$
\vec{P}=\chi_{l m}{ }^{0} E_{0} e^{i \omega_{i} !}+\chi_{I m}{ }^{1} u_{j 0} E_{0} e^{i\left(\omega_{i} \pm \omega_{,}\right) \prime}+\chi_{l m}{ }^{2} u_{j 0}{ }^{2} E_{0} e^{i\left(\omega_{i} \pm 2 \omega_{i}\right) \prime}+\cdots
$$

The first term is related to Rayleigh scattering in which the frequency of the radiation remains unchanged during the scattering process. The second term constitutes first-order Raman scattering. $\chi_{l m}{ }^{1}$ is called the Raman tensor, and corresponds to the first derivation of the polarizability term with respect to atomic displacement, Eq. 2.30. If the frequencies and wavevectors of incident and scattered light are $\left(\omega_{i}, \vec{k}_{i}\right)$ and $\left(\omega_{1}, \vec{k}_{1}\right)$ respectively, 
and $(\omega, \vec{k})$ of phonon is created or annihilated in the process. energy and momentum are conserved as follows:

$$
\begin{aligned}
& \hbar \omega_{,}=\hbar \omega_{i} \pm \hbar \omega \\
& \hbar \vec{k}_{,}=\hbar \vec{k}_{i} \pm \hbar \vec{k}
\end{aligned}
$$

In the above equations, the + and - sign denote anti-Stokes and Stokes scattering. respectively. The conservation of momentum reduces to the Bragg condition with $\omega_{i} \cong(1) j$ $>\omega$ approximation.

$$
k=2 k, \sin \frac{\phi}{2}=\frac{2 \omega_{1} n_{1}(\omega)}{c} \sin \frac{\phi}{2} .
$$

where $\phi$ is the angle between the incident and scattered directions and $n\left(\omega_{i}\right)$ is the index of refraction of the medium at $\omega_{\mathrm{i}}$. For visible light, $\left|\vec{k}_{i}\right|$ is about $10^{-4}$ to $10^{-3}$ of $\mathrm{k}_{\max }$ for phonons [44, 73]. Thus in a perfect crystal and for first-order Raman scattering. only phonons from the near center of the Brillouin zone are involved, i.e., $\vec{k} \approx 0$. This is a very important selection rule. This selection rule can be relaxed. Key cases are crystals with many impurities or when the incident light energy is near an interband electronic transition. The differential scattering cross-section per the scattering volume V for Stokes Raman scattering is given by

$$
\frac{d \sigma}{d \Omega}=\left.V^{\prime 2}\left(\frac{\omega_{1}{ }^{4}}{c}\right) \hat{\mathfrak{e}}_{i} \cdot \chi_{\ln }{ }^{\prime} \cdot \hat{\mathfrak{e}}_{1}\right|^{2}\left(\frac{1}{e^{\frac{\hbar_{1} \omega}{k_{B} T}}-1}\right)
$$

where $\hat{\mathrm{e}}_{\mathrm{i}}, \hat{\mathrm{e}}_{\mathrm{s}}$ are the unit polarization vectors of the incident and scattered light. respectively. 
We now consider atomic displacement polarization selection rules for diamond and zincblende structure materials. The Raman tensor for the $\vec{k}=0$ phonons for these materials is as follows [73].

$$
\left(\begin{array}{ccc}
0 & 0 & 0 \\
0 & 0 & d \\
0 & d & 0
\end{array}\right): u_{r_{1}} \quad\left(\begin{array}{ccc}
0 & 0 & d \\
0 & 0 & 0 \\
d & 0 & 0
\end{array}\right): u_{1_{1}} \quad\left(\begin{array}{ccc}
0 & d & 0 \\
d & 0 & 0 \\
0 & 0 & 0
\end{array}\right): u_{z=1}
$$

Each corresponds to phonons with atomic displacement along x. y. and z. respectively. For backscattering along $\mathrm{z}=(001)$ configuration, Raman selection rules can be calculated by using Raman tensors and Raman scattering cross-section equation as shown in Table 2.2. The selection rule applies to a crystal with full translational symmetry. This means that the crystal must be infinite. TO phonon propagates perpendicular to its vibrational direction. TO mode is forbidden for all configurations for (001) backscattering configuration.

When the energy of the incident light is near an interband electronic transition, there is strong absorption. The penetration depth. $L_{p}$, of the light is then given by

$$
L_{p} \approx \frac{2 \pi}{\Delta q}, \text { where } \Delta q=\operatorname{Im}\{\vec{k}\}+\operatorname{Im}\{\vec{k},\}
$$

In this case the light scattering is from a finite region of the material which does not have the full translational symmetry of the infinite crystal. Thus, there will be a change in the polarization selection rules due to this "linear $k$ " (LK) effect. Also, in the range of strong absorption, any surface electric field (SF) effects will become important, such as those originating from the space charge region. Electric fields also destroy the translational symmetry of the material. It is shown by group theory that the Raman tensor due to LK or 
Table 2.2: Atomic displacement Raman selection rules for backscattering from the (001) surface.

\begin{tabular}{|c||ccc|}
\hline Polarization & \multicolumn{4}{|c|}{ Phonon modes } \\
\cline { 2 - 4 } Configuration & $\mathrm{LO}\left(\mathrm{z}_{1}\right)$ & $\mathrm{TO}\left(\mathrm{x}_{1}\right)$ & $\overline{\mathrm{TO}}\left(\mathrm{y}_{1}\right)$ \\
\hline $\mathrm{z}_{1}\left(\mathrm{x}_{1}, \mathrm{x}_{1}\right)-\mathrm{z}_{1}$ & 0 & 0 & 0 \\
$\mathrm{z}_{1}\left(\mathrm{x}_{1}, \mathrm{y}_{1}\right)-\mathrm{z}_{1}$ & $\mathrm{~d}^{2}$ & 0 & 0 \\
$\mathrm{z}_{1}\left(\mathrm{y}_{1}, \mathrm{y}_{1}\right)-\mathrm{z}_{1}$ & 0 & 0 & 0 \\
\hline & $\mathrm{LO}\left(\mathrm{z}_{1}\right)$ & $\mathrm{TO}\left(\mathrm{x}^{\prime}{ }_{1}\right)$ & $\mathrm{TO}\left(\mathrm{y}^{\prime}{ }_{1}\right)$ \\
\hline & $\mathrm{d}^{2}$ & 0 & 0 \\
$\mathrm{z}_{1}\left(\mathrm{x}_{1}{ }^{\prime}, \mathrm{x}_{1}{ }^{\prime}\right)-\mathrm{z}_{1}$ & 0 & 0 & 0 \\
$\mathrm{z}_{1}\left(\mathrm{x}_{1}{ }^{\prime}, \mathrm{y}_{1}{ }^{\prime}\right)-\mathrm{z}_{1}$ & 0 & 0 & 0 \\
$\mathrm{z}_{1}\left(\mathrm{y}_{1}{ }^{\prime}, \mathrm{y}_{1}{ }^{\prime}\right)-\mathrm{z}_{1}$ & $\mathrm{~d}^{2}$ & 0 \\
\hline
\end{tabular}

Table 2.3: Surface field, linear $k$, and atomic displacement Raman selection rules for backscattering from the (001) surface.

\begin{tabular}{|c|c|c|c|}
\hline \multirow{2}{*}{$\begin{array}{c}\text { Polarization } \\
\text { Configuration }\end{array}$} & \multicolumn{3}{|c|}{ Phonon modes } \\
\hline & $\mathrm{LO}\left(\mathrm{z}_{1}\right)$ & TO $\left(x_{1}\right)$ & $\mathrm{TO}\left(y_{1}\right)$ \\
\hline$z_{1}\left(x_{1}, x_{1}\right)-z_{1}$ & $(b \varepsilon)^{2}$ & 0 & 0 \\
\hline$z_{1}\left(x_{1}, y_{1}\right)-z_{1}$ & $d^{2}$ & 0 & 0 \\
\hline$z_{1}\left(y_{1} \cdot y_{1}\right)-z_{1}$ & $(b \varepsilon)^{2}$ & 0 & 0 \\
\hline \multirow[b]{2}{*}{$z_{1}\left(x_{1}^{\prime}, x_{1}^{\prime}\right)-z_{1}$} & $\operatorname{LO}\left(z_{1}\right)$ & $\mathrm{TO}\left(\mathrm{x}_{1}^{\prime}\right)$ & TO $\left(y^{\prime}{ }_{1}\right)$ \\
\hline & $(d+b \varepsilon)^{2}$ & 0 & 0 \\
\hline $\mathrm{z}_{1}\left(\mathrm{x}_{1^{\prime}}, \mathrm{y}_{1}{ }^{\prime}\right)-\mathrm{z}_{1}$ & 0 & 0 & 0 \\
\hline$z_{1}\left(y_{1}^{\prime}, y_{1}^{\prime}\right)-z_{1}$ & $(d-b \varepsilon)^{2}$ & 0 & 0 \\
\hline
\end{tabular}

where

$$
\begin{aligned}
& x_{1}=(100) \quad y_{1}=(010) \quad z_{1}=(001) \\
& x_{1}{ }^{\prime}=\frac{1}{\sqrt{2}}(110) \quad y_{1}{ }^{\prime}=\frac{1}{\sqrt{2}}(1 \overline{1} 0)
\end{aligned}
$$


SF effects is the same. The tensor for the electric field $E_{\downarrow} . E_{y}, E_{z}$ are shown below. For LK effects, $k_{x}$ replaces $E_{x}$, etc. Then, Raman tensor for $S F$ and $A D$ scattering terms are $\left(\begin{array}{ccc}a E_{\mathrm{r}} & c E_{1} & c E_{z} \\ c E_{1} & b E_{x} & d \\ c E_{z} & d & b E_{x}\end{array}\right): u_{\mathrm{r}_{1}} \quad\left(\begin{array}{ccc}b E_{1} & c E_{x} & d \\ c E_{x} & a E_{1} & c E_{z} \\ d & c E_{z} & b E_{\mathrm{v}}\end{array}\right): u_{1_{1}} \quad\left(\begin{array}{ccc}b E_{z} & d & c E_{\mathrm{r}} \\ d & b E_{z} & c E_{\mathrm{y}} \\ c E_{\mathrm{r}} & c E_{1} & a E_{z}\end{array}\right): u_{z 1}$

Then. the Raman selection rule for (001) backscattering is modified as shown in Table 2.3 with $\mathrm{E}_{\mathrm{v}}=\mathrm{E}_{\mathrm{y}}=0$ and $\mathrm{E}_{\mathrm{z}}=\mathrm{E}$.

\subsection{Photoluminescence}

Photoluminescence (PL) spectroscopy is a direct way to non-destructively measure the band gap energy as a function of the carrier concentration [74-81] This technique measures the spectrum emitted by the radiative recombination of photogenerated minority carriers. PL intensity and lineshape are sensitive to crystalline quality and material composition [82]. PL study was done to get a better characterization of samples and to see if some complementary chemical level information could be obtained to compare with Raman carbon at arsenic LVM and carbon precipitation spectra.

In the simplest description, the optical process known as PL involves three major steps. First, an electron-hole pair is created with the absorption of incident light, resulting in a non-equilibrium condition in the material. The excited pair then seeks lower energy levels via thermalization. If the material is pure, the electron and holes pair off into excitons which then recombine. emitting a narrow spectral line at low temperatures. 
However at temperatures such that $\mathrm{k}_{\mathrm{B}} \mathrm{T}>($ exciton ionization energy). and also in less pure crystals, the free carriers can then recombine radiatively in a band-to-band transition and/or deep transition. The deep transition stands for the transition of an electron from the conduction band to an acceptor state or a transition from a donor to the valence band. If a direct gap between parabolic bands was assumed and the impurities were taken to be shallow. discrete, and nonoverlapping. the band-to-band transition would be about four times more probable than the transition between the impurity (donors or acceptors) and the band [9]. However when the impurity concentration is large enough to form an impurity band which merges with the nearest intrinsic band, the interpretation of the process becomes ambiguous. When electric dipole transitions are allowed, the electronhole pair recombines with the emission of a photon with high probability [12.83]. The transition rate, $W_{t \rightarrow f}$. from the initial state $i$ to the final state $f$ is governed by Fermis Golden rule;

$$
W_{l \rightarrow f}=\frac{2 \pi}{\hbar}\left|H_{f i}^{\prime}\right|^{2}\left\{\delta\left(E_{,}-E_{l}-\hbar \omega\right)+\delta\left(E_{f}-E_{i}+\hbar \omega\right)\right\}
$$

where $E_{f}\left(E_{i}\right)$ stands for the energy of final (initial) state, and $+(-) \hbar \omega$ is the energy of absorbed (emitted) light. $H_{t \prime}^{\prime}$ is the transition matrix element given by

$$
H_{1 \prime}^{\prime}=\left\langle\varphi_{t}\left|H^{\prime}\right| \varphi_{1}\right\rangle
$$

where $\varphi_{\mathrm{f}}\left(\varphi_{\mathrm{i}}\right)$ represents the final (initial) state function, and $\mathrm{H}^{\prime}$ is a perturbation term in the Hamiltonian function. Fermi's Golden rule is derived from time dependent perturbation theory for the radiation field and atomic system [82-85]. The transition rate is also controlled by momentum conservation. For direct transition; 


$$
\vec{q}+\vec{k}_{\imath}=\vec{k}_{1}
$$

where $\vec{k}_{i}\left(\vec{k}_{,}\right)$is the wave vector of electrons in initial (final) state, and $\vec{q}$ is that of incident or emitted light. Usually $|\vec{q}|$ is very small compared to $\left|\vec{k}_{l}\right|$ and $\left|\vec{k}_{f}\right|$. resulting in $\left|\vec{k}_{i}\right| \approx\left|\vec{k}_{f}\right|$ which means transition is direct. Finally, the recombination radiation escapes from the sample. The electron-hole pair could also recombine via non-radiative recombination paths, which compete with the radiative transition path [7]. The generation of electron-hole pairs in semiconductors involves the transition of carriers across the forbidden gap and thus is very different for direct and indirect gap material.

The spontaneous band-to-band emission rate can be obtained on the basis of the thermodynamic equilibrium condition, and the emission spectrum $S(E)$ is expresses as $[86,87]$

$$
S(E)=\frac{8 \pi n e^{2} E^{2} \alpha(E)}{h^{3} c^{2}\left(e^{\frac{l}{k_{3} T}}-1\right)}
$$

where $n$ and $\alpha(E)$ represent the index of refraction and absorption coefficient. respectively. The absorption coefficient $\alpha(E)$ is proportional to density of state, therefore proportional to $\sqrt{E-E_{g}}$ for allowed direct transitions. $\mathrm{E}_{\underline{g}}$ stands for the band gap which is a function of temperature. The band gap of pure GaAs shows the quadratic dependence on the temperature which is characteristic of direct band gap material according to Varshni equation [88] and shown as follows [89].

$$
E_{x . d i r}(T)=1.519-\frac{5.408 \times 10^{-4} T^{2}}{T+204}
$$


From the above equation, band gap of pure GaAs at $77 \mathrm{~K}$ is $1.508 \mathrm{eV}$. The emission spectrum from pure GaAs at room temperature shows intense band peaking near $E_{g}$ with the shape described above as shown in Fig. 2.9 (a) [90]. W'ith photoluminescence of pure GaAs at $77 \mathrm{~K}$, emission spectra of $n$ - (Te doped) and $p$ - ( $\mathrm{Zn}$ doped) type GaAs are also included in Fig. 2.9 (b) for comparison.

For $n$-type GaAs in Fig. 2.9 (b). the peak of PL is blue shifted as carrier concentration increases. This is attributed to Burstein-Moss shift [91. 92]. i.e.. a filling of the conduction band by electrons. The peak extends to lower energy as carrier concentration increases. This is due to energy gap renormalization and bandtailing. As carrier concentration increases, the conduction band edge moves downward and the valence band edge moves upward resulting in band gap renormalization. This is caused by carrier impurity ions interaction and by many-body effect [77. 93]. The many-body effects result from the spin-spin interaction and Coulomb repulsive interaction between the free carriers. The band gap renormalization as a function of carrier concentration can be represented as follows:

$$
\Delta E_{g}=E_{g}(\text { doped })-E_{g}(\text { pure })=-A n^{1 / 3} .
$$

The coefficient $\mathrm{A}$ has been adjusted to give the measured value of $\Delta \mathrm{E}_{\mathrm{g}}$ at high dopant concentrations, and the minus sign means that the band gap shrinks with increasing impurity concentration. Borghs et al. [77] observed $\mathrm{A}=-7.3 \times 10^{-8} \mathrm{eV} \cdot \mathrm{cm}$ from their data fitting. The bandtailing is caused by the broadening and merging of the impurity energy levels with intrinsic bands. If impurity atoms are introduced, they may form discrete energy levels in the forbidden band. If their wave functions overlap, the impurity levels begin broadening. Then the allowed energy region, the impurity band. arises in the 


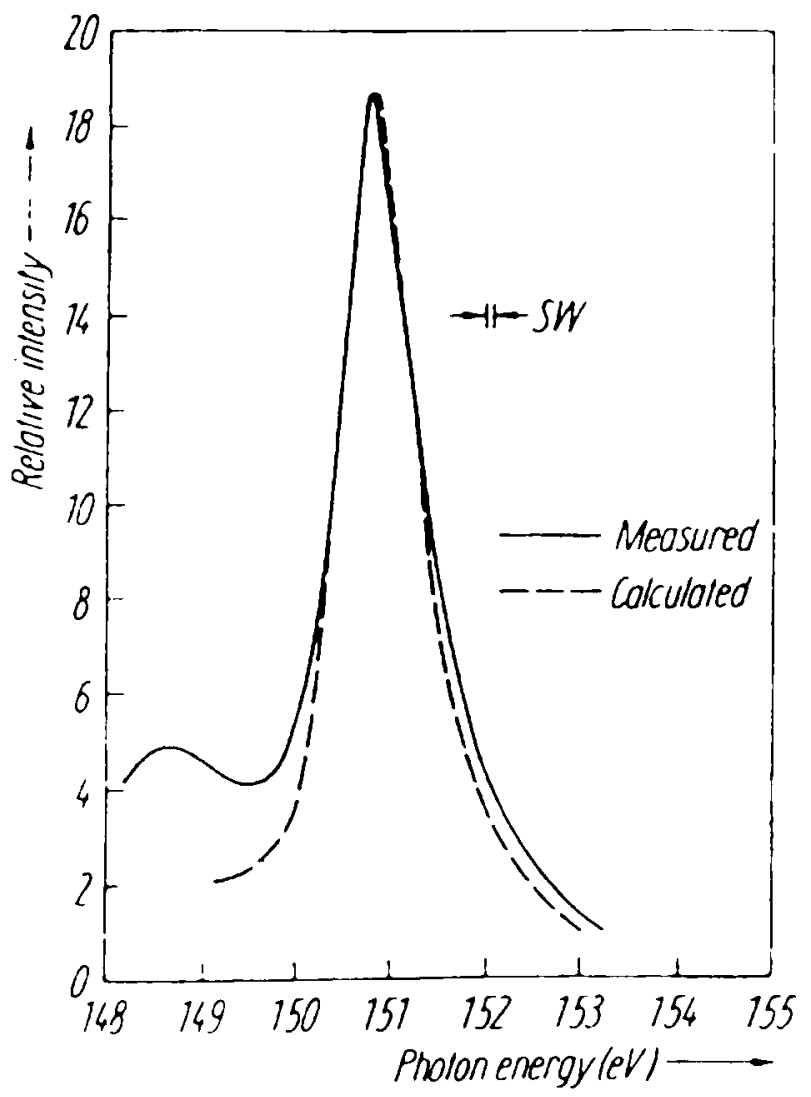

(a)

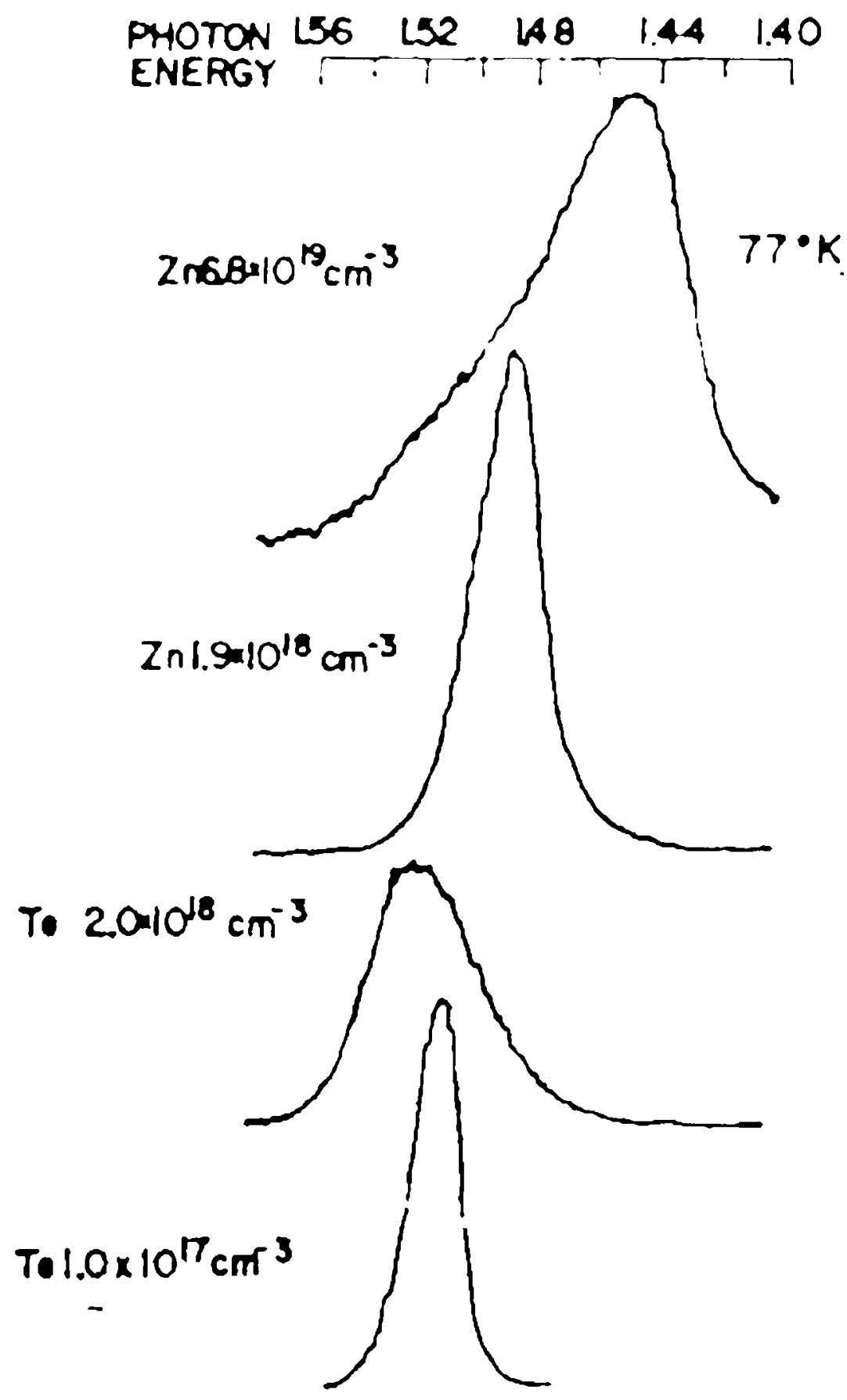

(b)

Figure 2.9: Photoluminescence spectra of (a) pure GaAs [90] and (b) n-type Grit: $:$ : $\Gamma_{c}$ and p-type GaAs:Zn [94] at $77 \mathrm{~K}$. 
forbidden gap. The case when there is an impurity band separated both from the conduction and valence bands of the host crystal is referred to as intermediate doping. As impurity concentration increases, the impurity band becomes broader, resulting in decrease of the activation energy determining the electron (hole) concentration in the conduction (valence) band. Finally, when the host material is heavily doped, the activation energy is zero. This means that the top (bottom) of the donor (acceptor) impurity band merges into the conduction (valence) band. The net result is an apparent bandgap shrinkage. The full width half maximum of the peak is increased as carrier density increases. This is attributed to Burstein-Moss shift, band gap renormalization, and bandtailing.

For p-type GaAs in Fig. 2.9 (b), the peak of PL is red shifted as carrier concentration increases. For GaAs, the higher hole effective mass must shrink the band gap more in $p$-type material than in $n$-type material [95]. In $p$-type emission, the shift to higher energy due to the Burstein-Moss effect is masked by the energy gap decrease with increasing concentration [77, 94]. The hole concentration dependence of the band gap shrinkage in p-type GaAs can be represented as follows:

$$
\Delta E_{g}=E_{g}(\text { doped })-E_{g}(\text { pure })=-A p^{1 / 3}
$$

$A$ is suggested by many research groups and ranging between $1.6 \times 10^{-8}$ and $2.6 \times 10^{-8}$ $\mathrm{eV} \cdot \mathrm{cm}[77,80,96]$.

\subsection{Annealing}

Post-deposition processing may include both standard and rapid annealing treatments. With this device processing, device operation might introduce high 
temperature. The elevated temperatures are capable of altering compound semiconductor stoichiometry. crystal quality and microscopic defect structure unexpectedly. To better understand these phenomena. I conducted several anneals and studied the microscopic effects through Raman spectra.

It has been reported that after carbon doped GaAs annealing at low temperatures $\left(\mathrm{T}_{\mathrm{a}}<550{ }^{\circ} \mathrm{C}\right)$. the hole concentration increases [97]. This is attributed to the decrease in the concentration of hydrogen in the sample [97-99]. Secondary ion mass spectroscopy (SIMS) profiles reveal a corresponding decrease in $[\mathrm{H}]$ as a function of annealing temperature. $T_{a}[97,99]$. SIMS is a mass analysis technique to provide a quantitative indication of the sample surface s composition by using secondary ions from the sample surface. Hole concentration reduction in heavily carbon doped GaAs upon high temperature annealing $\left(\mathrm{T}_{\mathrm{a}}>600^{\circ} \mathrm{C}\right)$ has been reported by numerous authors [26. 28. 97. 100-102]. Higher carrier density samples show sharper decrease. A significant decrease in the hole concentration occurs at the early stage of the anneal. and then a decrease slows down as the anneal proceeds [101]. The carrier concentration was found to reach a minimum concentration. $p_{m m n}$. independent of as grown $[\mathrm{C}][97.101 .103]$. Each research group observed different $p_{m i n}$. Westwater et al. [103] and Höfler et al. [97] found $p_{\min } \approx$ $(4 \sim 5) \times 10^{19} \mathrm{~cm}^{-3}$. Not every annealing study on carbon doped GaAs shows the behavior mentioned above. Carrier concentration increase is observed for heavily carbon doped epilayers $\left(4.7 \times 10^{19}\right.$ and $\left.9.8 \times 10^{19} \mathrm{~cm}^{-3}\right)$ by rapid annealing from $500{ }^{\circ} \mathrm{C}$ to $900{ }^{\circ} \mathrm{C}[99]$. These differences are attributed to a difference in annealing conditions or growth techniques. No carbon concentration decrease is observed by SIMS measurement [28. 98. 99. 101. 103]. Therefore, the observed $p$ reduction is not due to passivation by loss of $\mathrm{C}$. 
Many mechanisms are suggested to explain the observed decrease in $p$ and mobility upon high temperature annealing, as follows:

1. $\mathrm{C}_{\mathrm{As}}^{-} \rightarrow$ interstitial $\mathrm{C}_{\mathrm{i}}[97,98,100,101,104]:$

The site switching of carbon atoms from a substitutional site to an interstitial site leaving behind an arsenic vacancy might cause $p$ reduction upon annealing. These interstitial defects might be electrically neutral or part of a complex defect or precipitate. This argument can be verified by comparing the number of carbon interstitials before and after annealing. This can be done by ion-beam channeling studies as Höfler et al. [97]. Their study showed that the fraction of interstitial $\mathrm{C}$ after annealing at $\mathrm{T}_{\mathrm{a}}>600{ }^{\circ} \mathrm{C}$ is at least $70 \%$ higher than in the as grown samples. Carbon interstitial can be identified by dicarbon Raman signal observed by Wagner et al. [105]. As for the carbon interstitial. formation of di-carbon centers is deduced from the observation of Raman lines at $17+2$. 1708 , and $1674 \mathrm{~cm}^{-1}$ in GaAs co-doped with ${ }^{12} \mathrm{C}$ and ${ }^{13} \mathrm{C}$ after annealing at $850{ }^{\circ} \mathrm{C}$ with concomitant loss of vibrational scattering from $C_{A s}[105]$. The frequencies agree with results of theory for a $\mathrm{C}-\mathrm{C}$ formed by the trapping of a mobile interstitial $\mathrm{C}$ (displaced $\mathrm{C}_{\mathrm{As}}$ ) atom by an undisplaced $\mathrm{C}_{\mathrm{As}}$ acceptor. The proposed structure of this complex is shown in Fig. 2.10. Other mechanisms of carrier loss are inferred since a weaker Raman triplet is detected at 1859,1824 , and $1788 \mathrm{~cm}^{-1}$ from a different $\mathrm{C}-\mathrm{C}$ complex.

2. $\mathrm{C}_{\mathrm{As}}{ }^{-} \rightarrow$ precipitatates $[28,97,101-103,106]:$

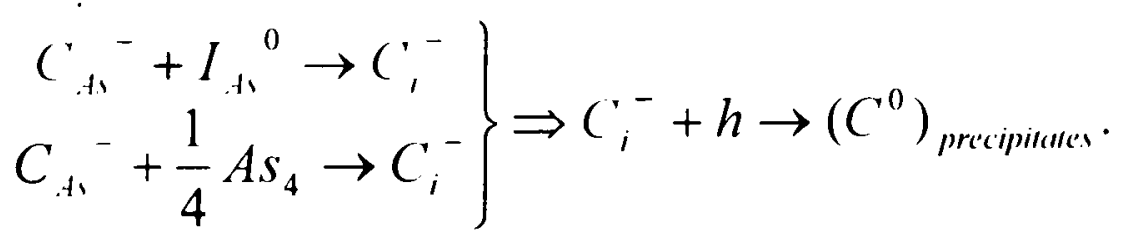




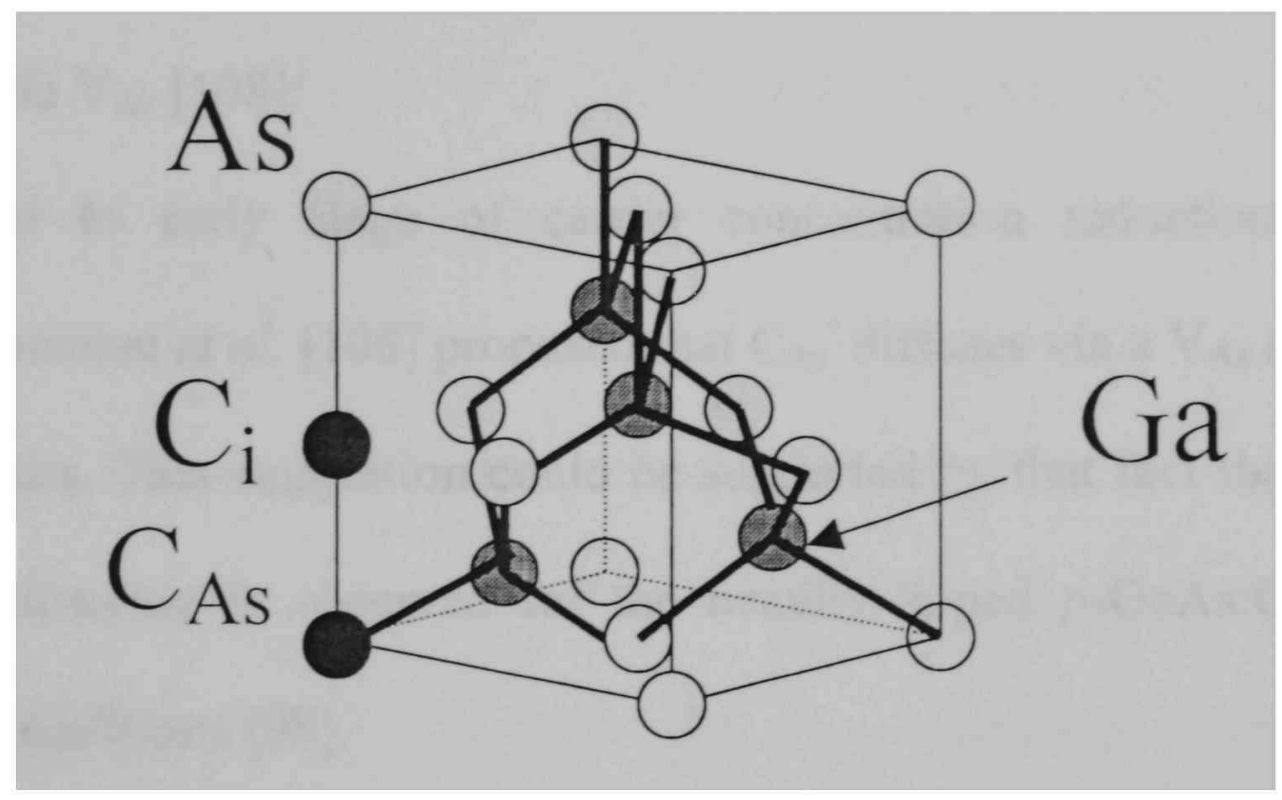

Figure 2.10: Di-carbon $\left(\mathrm{C}_{\mathrm{i}}-\mathrm{C}_{\mathrm{As}}\right)$ defect structure proposed by Wagner et al. [105]. 
The initial reduction in carrier concentration and mobility after anneals is a result of supersaturated $\mathrm{C}_{\mathrm{As}}$ moving to interstitial sites. As annealing temperature and time increase, clustering of $\mathrm{C}$ leads to the formation of precipitates. If the size of the cluster is big enough, $\mathrm{C}-\mathrm{C}$ bonds will show up in Raman peaks at $\sim 1355 \mathrm{~cm}^{-1}$ and $\sim 1585 \mathrm{~cm}^{-1}$.

3. Site switching: $\mathrm{C}_{\mathrm{As}} \rightarrow \mathrm{C}_{\mathrm{Ga}}{ }^{+}[26]$ :

Migration of carbon from As site to Ga site upon annealing might cause the $p$ reduction. The carbon at gallium site LVM should be infrared active and is expected near $C_{A}$ LVM, in the range of $480 \sim 500 \mathrm{~cm}^{-1}$ [107] from the analogy of the LVM frequencies for ${ }^{28} \mathrm{Si}_{\mathrm{i}_{\mathrm{ia}}}$ donors at $384 \mathrm{~cm}^{-1}$ and ${ }^{28} \mathrm{Si}_{\mathrm{As}}$ acceptors at $399 \mathrm{~cm}^{-1}$.

4. Diffusion via $\mathrm{V}_{\mathrm{As}}[108]$ :

This is related to early stage of carrier concentration reduction. As a diffusion mechanism. Uematsu et al. [108] proposed that $C_{A s}$ diffuses via a $V_{A s}$ species in the form of $\left(\mathrm{C}_{\mathrm{As}} \mathrm{V}_{\mathrm{As}}\right)^{-}$pairs. This suggestion could be supported by that fact that the smaller hole concentration decrease is observed for the heavily doped $p$-GaAs: $\mathrm{C}$ epilayers grown under As rich conditions [98].

5. Passivation by the incorporation of hydrogen [34]:

The passivation of carbon acceptors by hydrogen in GaAs is observed by Pan et al. [109]. They proposed the following passivation process. The valence electron of the atomic hydrogen recombines with a free hole leaving behind a proton. Because there is Coulombic attraction between the proton and the negatively charged $\mathrm{C}$ acceptor. pairing occurs resulting in an electrically deactivated $\mathrm{C}$. The hydrogen might be introduced during the growth of layers by MOVPE, MOMBE, or CBE from metalorganic sources. The paired hydrogen atom gives rise to a high-frequency asymmetric LVM 
corresponding to stretching of the bond to its nearest-neighbor atoms [110]. The LVM of $\mathrm{H}-{ }^{12} \mathrm{C}_{\mathrm{As}}$ pair. $\mathrm{H}-{ }^{12} \mathrm{C}_{\mathrm{As}}$ stretching mode, identified at $2636 \mathrm{~cm}^{-1}$ by Woodhouse et al. [34] was used to verify the existence of the pair. The H-C $\mathrm{C}_{\mathrm{As}}$ mode can $\mathrm{t}$. however, be used in explaining hole concentration reduction under annealing if hydrogen concentration introduced during the growth of layers is under SIMS detection limit.

6. Formation of misfit dislocations [100]:

Dislocations can acts as carrier traps and reduce the efficiency of radiative recombination (PL intensity decrease upon annealing will be discussed in section 4.2.), and therefore, may explain the degradation in electrical and optical properties [100. 107]. Partial relaxation of the C-doped layers is attributed to the introduction of misfit dislocations [103]. This introduction of misfit dislocation occurs only at thicknesses significantly above the critical thickness [111].

On the contrary to carrier concentration, mobility stays almost constant except in very high carrier concentration range, $p>6 \times 10^{19} \mathrm{~cm}^{-3}[97.100]$. At very high carrier concentration range, the mobility decreased after annealing. With $p$ and mobility reduction upon annealing. lattice contraction decrease is also observed $[97,100,104]$. The origin of the decrease in lattice contraction is not understood clearly. This might be due to the consequence of a solid solubility limit for substitutional carbon and a resulting site switching to interstitial sites [97]. One of other possibilities is due to strain relaxation via misfit dislocation [100]. Even though each process mentioned above can explain hole denisity reduction, many processes should be combined to explain the whole annealing effect. For example, even though precipitation explains observed $p$ reduction, SIMS data and carbon precipitation Raman peaks. it does not explain the mobility reduction upon 
annealing because the total density of ionized impurities..$V_{\%}$. does not change or decrease in precipitation model. For degenerate semiconductors. the mobility is inversely proportional to the number of ionized impurities, $\mu \sim p / \Lambda_{i}[100.112]$. Therefore. precipitation is not the sole contributor to the reduction of free hole concentration. For site switching model $\left(\mathrm{C}_{\mathrm{As}} \rightarrow \mathrm{C}_{\mathrm{Ga}}{ }^{+}\right)$, no direct evidence, like $\mathrm{C}_{\mathrm{Ca}_{\mathrm{a}}}$ LVM. is observed in Raman or infrared spectroscopy [103]. Further, it cannot explain the large reduction observed in the lattice contraction of the carbon-doped epilayer $[97,100,104]$. For diffusion via $\mathrm{V}_{\mathrm{As}}$ mechanism, it does not explain the higher $\mathrm{C}_{\mathrm{As}}{ }^{-}$diffusivity increases for higher $\mathrm{As}_{4}$ pressure upon annealing [28]. For the passivation by the incorporation of hydrogen (mechanism 5 above), even though $\mathrm{H}_{-}{ }^{12} \mathrm{C}_{\mathrm{As}}$ mode is observed from annealed GaAs:C [34]. this possibility is rejected by many groups. Their argument is based on $[\mathrm{H}]$ level decreases observed in samples annealed $T_{a}>650{ }^{\circ} \mathrm{C}[97,99]$. The hole concentration is decreased even though most hydrogen is evacuated by thermal annealing, indicating that the hole decrease is not related to hydrogen passivation [98]. Westwater et al. [103] observed $p$ decrease after high temperature annealing without observing any sign for $\mathrm{C}-\mathrm{H}_{\mathrm{As}}$ complex before and after annealing.

From this discussion, it is clear that annealing effects on heavily carbon doped GaAs are not completely understood. In this study, Raman spectroscopy and photoluminescence are used to add understanding about hole concentration. mobility reduction and lattice strain relaxation upon annealing. 


\section{CHAPTER III \\ EXPERIMENTAL DETAILS}

\subsection{Sample Information}

The carbon doped GaAs samples studied here are provided by T. Kim and W.D. Duncan at Central Research Laboratory at Texas Instruments. Inc. Samples were grown by organo-metallic chemical vapor deposition (OMCVD) on pure, (001) GaAs substrates in EMCORE GS-3200 organometallic vapor-phase epitaxy system. CVD is one of the most widely used techniques of crystal growth, and refers to the formation of a condensed phase from a gaseous medium of different chemical composition [7, 113. 114]. The OMCVD has emerged as a technologically important one for the production of single layers, heterojunctions, and quantum well structures due to its excellent control over layer thickness and doping, and the achievement of hyperabrupt junctions [23. 113. $115,116]$. As source gases. trimethylgallium $\left(\mathrm{Ga}\left(\mathrm{CH}_{3}\right)_{3}\right)$, tetrabutylarsine $\left(\left(\mathrm{CH}_{3}\right)_{3} \mathrm{CAsH}_{2}\right)$ are used. Controlled carbon doping was achieved by using carbon tetrachloride $\left(\mathrm{CCl}_{4}\right)$ as the external carbon source. Even though some of $\mathrm{C}$ atoms from TMGa are incorporated in GaAs, the carbon doping level control with TMGa as a sole carbon source is not easy since V:III ratio and temperature not only changes the doping level. but also growth rate and morphologies. Thus, it is convenient to have a separate doping source. $\mathrm{CCl}_{4}$ is used as a separate dopant source for the samples studied in here. To achieve high doping concentrations, low growth temperature $\left(<600^{\circ} \mathrm{C}\right)$ and V:III molar-flow rate ratios $(<$ 10) were maintained. Carrier concentrations were determined by Hall effect measurements. The hole concentrations range from $2.3 \times 10^{19}$ to $1 \times 10^{20} \mathrm{~cm}^{-3}$. The 
epilayer thicknesses of the samples were $\approx 100 \mathrm{~nm}$. This is thicker than the penetration depth, which is the inverse of the absorption coefficient $\alpha$, for the excitation light used for photoluminescence (PL) and Raman measurements. Therefore, PL and Raman signal from the samples are mainly from the epilayer, rather than from the substrate. More information on samples is shown in Table 3.1.

\subsection{Photoluminescence}

Photoluminescence spectra were generated with $488 \mathrm{~nm}$ argon-ion laser light (Coherent Innova 300). A diffraction grating filter (Optometrics TGF-302) was used to remove unwanted plasma lines. This filter has a band pass of $2 \mathrm{~nm}$. The incident and scattered light are directed and collected by mirrors and lenses as shown in Fig. 3.1. The samples were immersed in the liquid nitrogen during the measurements. A scanning spectrometer (ISA HR 320) with 1200 grooves per $\mathrm{mm}(\mathrm{gr} / \mathrm{mm})$ grating (optimized at $900 \mathrm{~nm}, 68 \mathrm{~mm} \times 68 \mathrm{~mm}$ ) was used to disperse the scattered light, corresponding to a spectral resolution of $\approx 0.2 \AA \approx 0.07 \mathrm{meV}$. Slit width was set between 200 and 300 micrometer. Signal from the germanium detector (EG\&G Judson-JD16 model) was amplified by a lock-in amplifier (SRS SR 830). Lock-in amplifiers use a technique known as phase-sensitive detection to single out the component of the signal at a specific reference frequency and phase. Here the reference frequency from the chopper and phase are $209 \mathrm{~Hz}$ and $90^{\circ}$. This lock-in amplifier technique is good for detecting very small signal. All spectra were corrected for the wavelength dependent system response. Details of the PL setup are described in Ref. 117. 
Table 3.1: Heavily carbon doped GaAs sample information

\begin{tabular}{|c|cccc|}
\hline Run\# & $\begin{array}{c}\text { Thickness } \\
(\mathrm{nm})\end{array}$ & $\begin{array}{c}\text { Carrier } \\
\text { concentration } \\
\left(\mathrm{P} / \mathrm{cm}^{3}\right)\end{array}$ & $\begin{array}{c}\text { Sheet } \\
\text { Resistance } \\
\mathrm{R}(\Omega /)\end{array}$ & $\begin{array}{c}\text { Mobility } \\
\mu\left(\mathrm{cm}^{2} / \mathrm{Vs}\right)\end{array}$ \\
\hline $\mathrm{E} 6303$ & 94.905 & $2.3 \mathrm{E}+19$ & $4.25 \mathrm{E}+02$ & 67.53 \\
$\mathrm{E} 6301$ & 94.905 & $2.9 \mathrm{E}+19$ & $2.76 \mathrm{E}+02$ & 82.59 \\
$\mathrm{E} 6314$ & 94.905 & $4.29 \mathrm{E}+19$ & $2.28 \mathrm{E}+02$ & 67.45 \\
$\mathrm{E} 6331$ & 94.905 & $4.62 \mathrm{E}+19$ & $1.93 \mathrm{E}+02$ & 73.89 \\
$\mathrm{E} 6302$ & 93 & $4.87 \mathrm{E}+19$ & $1.87 \mathrm{E}+02$ & 73.98333 \\
E6315 & 94.905 & $5.31 \mathrm{E}+19$ & $1.90 \mathrm{E}+02$ & 65.45 \\
E6269 & 93 & $7.54 \mathrm{E}+19$ & $1.31 \mathrm{E}+02$ & 68.18333 \\
E6349 & 1 & $\mathrm{E}+20$ & & \\
\hline
\end{tabular}




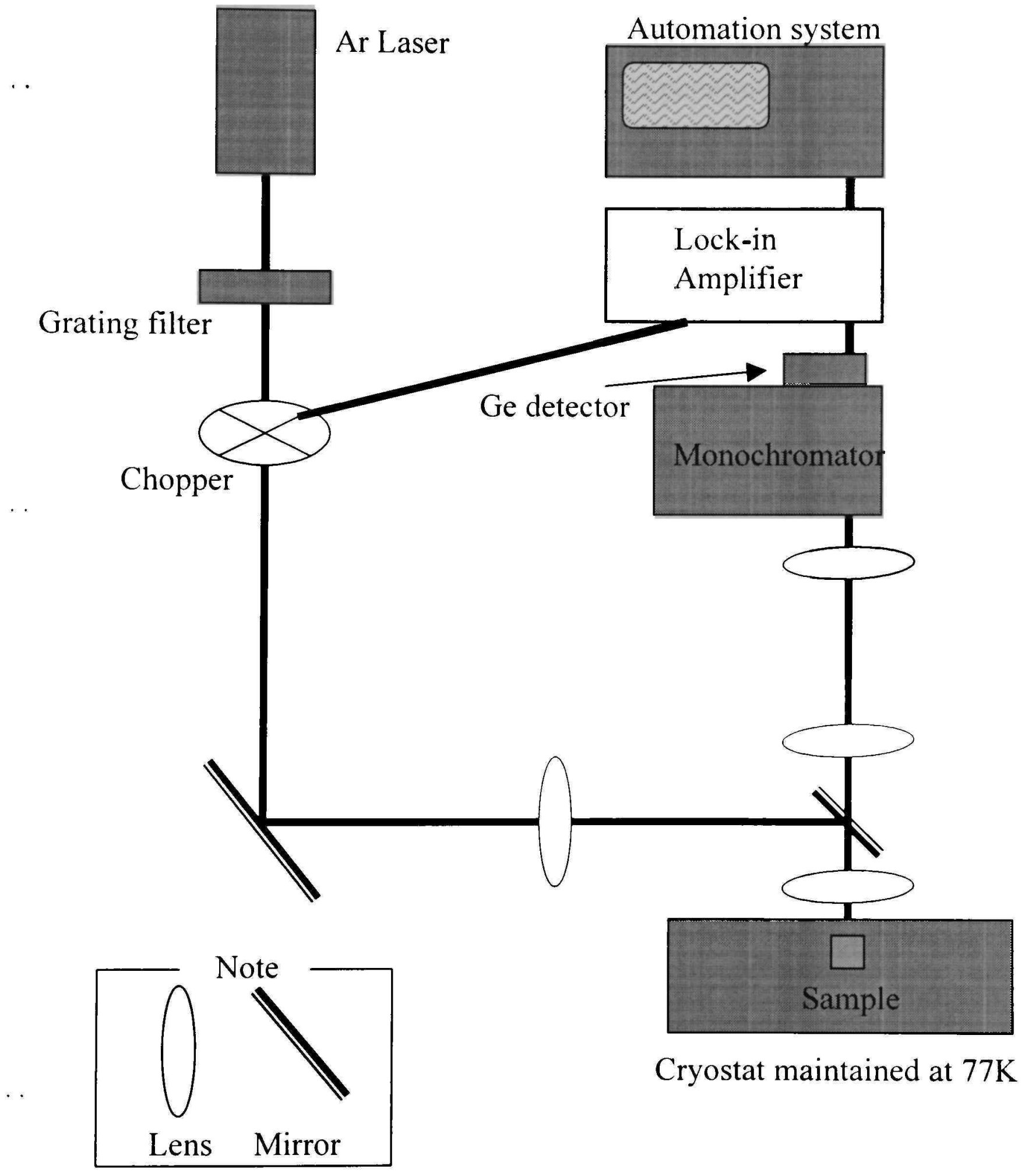

Figure 3.1: Photoluminescence setup. 


\subsection{Raman Spectroscopy}

Raman measurements were performed in backscattering geometry from the $(001)$ growth surface with the samples at room temperature. The spectra were excited with the $2.71 \mathrm{eV}(457.9 \mathrm{~nm}), 2.54 \mathrm{eV}(488.0 \mathrm{~nm})$ and $2.41 \mathrm{eV}(514.5 \mathrm{~nm})$ lines of an argon-ion laser (Coherent Innova 300). For each excitation line, the optical penetration depth is ₹ $26, \approx 45, \approx 55 \mathrm{~nm}$, respectively [118]. The optical penetration depth is the distance from the surface of a sample which the intensity of the incident light has fallen $1 / \mathrm{e} \approx 37 \%$ of its intensity. Because the scattered light must escape from the surface material. the optical penetration depth is $1 /(2 \alpha)$ where $\alpha$ is an absorption coefficient of incident light. Excitation light from the laser passes through the grating filter to get rid of plasma lines. The incident and scattered light are directed and collected by mirrors and lenses as shown in Fig. 3.2. Standard optical components were used to control polarization conditions. The laser light is focused to $\mathrm{a} \approx 10 \mu \mathrm{m}$ diameter spot using a SPEX MicraMate microRaman instrument. The surface of the sample on the xyz stage can be viewed using a camera and monitor. The magnification of the image on the monitor is about 1000 . Due to this system, careful study of the surfaces of the annealed samples was possible. Notch filters are used to remove Rayleigh scattering due to elastic scattering. The notch filters attenuates the Rayleigh scattering, allows $80 \%$ of other wavelengths through, and makes this type of multichannel system possible. The filters have a bandwidth of $<20 \mathrm{~nm}(<$ $\left.700 \mathrm{~cm}^{-1}\right)$. The notch filters can be rotated in a plane orthogonal to the incoming light to shift the cutoff edge. The scattered light is dispersed by 0.5 meter monochromator (SPEX $500 \mathrm{M}$ ) whose input slit is set to $200 \mu \mathrm{m}$. For Raman shift, $\Delta v$, smaller than $1000 \mathrm{~cm}^{-1}$. 


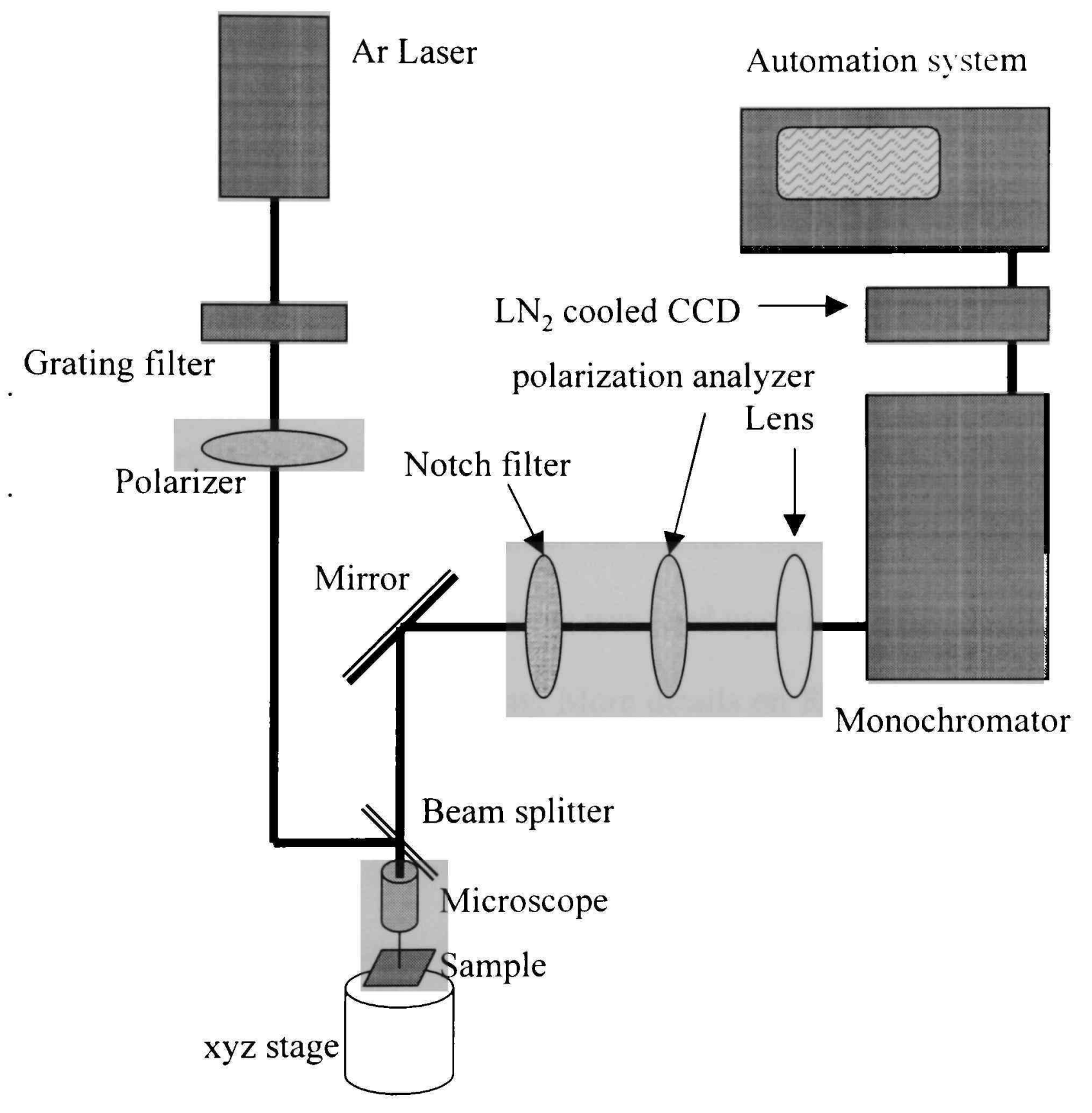

Figure 3.2: MicroRaman setup for Raman measurements. 
$1800 \mathrm{gr} / \mathrm{mm}$ grating (optimized at $500 \mathrm{~nm} .110 \mathrm{~mm} \times 110 \mathrm{~mm}$ ) is used. and $1200 \mathrm{gr} / \mathrm{mm}$ grating (optimized at $500 \mathrm{~nm}, 110 \mathrm{~mm} \times 110 \mathrm{~mm}$ ) is used for $\Delta v>1000 \mathrm{~cm}^{-1}$. Even though larger number of lines per mm grating gives better resolution. lower number of lines per mm grating was used for $\Delta v^{\prime}>1000 \mathrm{~cm}^{-1}$ to have enough spectral coverage. A liquid nitrogen cooled multichannel charge-coupled device (Princeton Instruments, LNCCD-512 TKB) was used to detect the scattered light. The Raman shift range of the detector is calibrated using either known emission lines from lamps (neon or mercury) or plasma lines from the laser. The CCD has a $512 \times 512$ pixel grid with an active area of $1.2 \mathrm{~cm}^{2}$ and the chip is maintained at a temperature of $110^{\circ} \mathrm{C}$ to minimize the thermal dark current. The dark current occurs due to charge leakage within each pixel and is exponentially related to the temperature. The ADC (analog-digital convert) limit is 65536 (16 bit) and is read out sequentially after the allotted exposure time has elapsed. CSMA software written by Princeton Instruments was used to control data taking parameters and manage the read-out of the CCD array. More details on Raman setup used in this study are described in Ref. 119. Integration times ranged from several minutes to three hours. Even though long integration time offers theoretically better signal-to-noise $(\mathrm{S} / \mathrm{N})$ ratio. cosmic rays limit the $\mathrm{S} / \mathrm{N}$ ratio improvement with long integration times. Therefore for long integration time Raman spectrum, many short Raman spectra are taken sequentially'. rather than just one long spectrum. and processed by computer program specifically designed to reduce these effects especially for very low scattered signal conditions. The program is written based on Adjouri et al.'s article [120]. The main steps of the data analysis are spike removal, standard statistical testing and enhancement of the mean 
statistical accuracy. In this program, a number of data files are read and a median value for each channel, $\mathrm{m}_{\mathfrak{c}}$, is calculated. Then, the intensity of each channel is compared with

$$
m_{c} \pm\left(\alpha_{1}+\beta_{1} \sqrt{m_{c}}\right)
$$

where $\alpha_{1}$ and $\beta_{1}$ are two constants adjusted to experimental conditions. In this step, using median value, not mean value, is very important because the mean value of the intensity accumulation may be modified by excessively high values generated by cosmic rays. For each spectra, if the intensity of each channel lies outside of the range given by Eq. 4.1, the data point is rejected. After first spike removal, each spectrum is normalized. Normalization step is introduced to standardize the statistical data processing procedure. This normalization step goes next to first spike removal process. Otherwise, at some spectra, data might be normalized with respect to cosmic rays because cosmic ray spikes are usually very intense compared to that of Raman signal. After normalization, second spike removal is conducted. The second spike removal step is introduced to get rid of weaker spikes.

$$
m_{c} \pm\left(\alpha_{2}+\beta_{2} \sqrt{m_{c}}\right)
$$

where $\alpha_{2}$ and $\beta_{2}$ are smaller than $\alpha_{1}$ and $\beta_{1}$. Finally, the obtained spectra undergo a statistical test procedure. For each channel, the mean value $\mu_{\mathrm{c}}$, and standard deviation $\sigma_{\mathrm{c}}$ of the remaining intensity values are calculated and an interval $\left(\mu_{c}-\xi \sigma_{c}, \mu_{c}+\xi \sigma_{c}\right)$ is defined. $\xi$ is a constant. Parameters, $\alpha_{1}, \beta_{1}, \alpha_{2}, \beta_{2}$, and $\xi$, are selected to get a low data rejection rate and a good $\mathrm{S} / \mathrm{N}$ ratio. Again, data fall outside of the interval for each channel will be discarded. The remained data are averaged and saved. The number of 
remained data per each channel and averaged data are displayed. and these plots are used to diagnose parameters used, $\alpha_{1}, \beta_{1}, \alpha_{2}, \beta_{2}$, and $\xi$.

\subsection{Annealing}

The annealing system shown in Fig. 3.3 was used in Maddox Lab to stud! the effect of elevated temperatures comparable to what is used in device processing. The $\mathrm{N}_{2}$ gas flow rate was about 300 standard cubic $\mathrm{cm}$ per minute. To minimize As surface loss during annealing, samples are loaded in face to face geometry [103, 121]. referred to as a proximity cap, and surrounded by pure GaAs. This provides an environment in which the As pressure gradient at the sample surface is minimized. The annealing temperature ranges from $450^{\circ} \mathrm{C}$ to $850^{\circ} \mathrm{C}$. and annealing time from 20 minutes to 1 hour. 


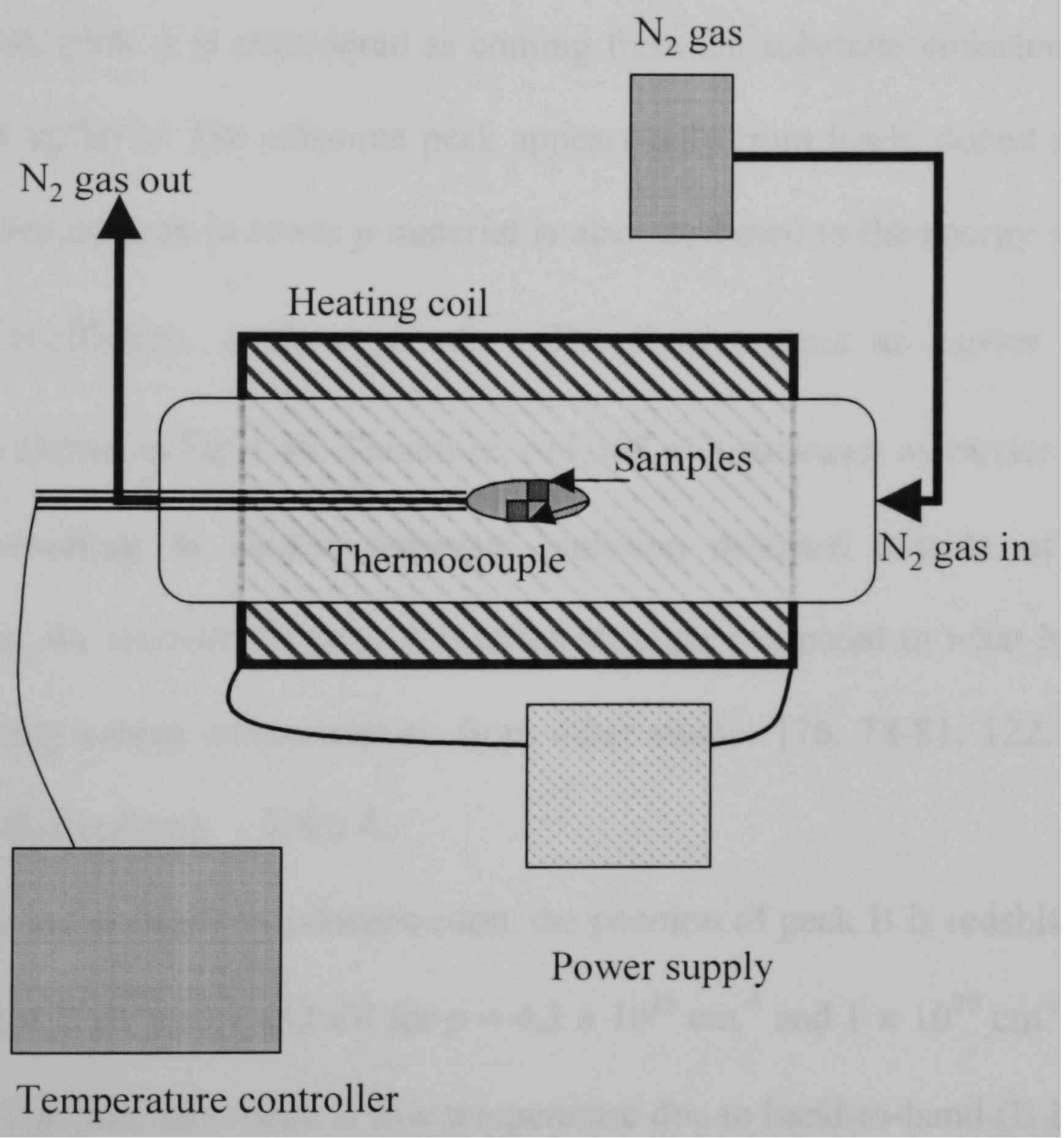

Figure 3.3: Annealing System 


\section{CHAPTER IV}

\section{PHOTOLUMINESCENCE OF HEAVILY CARBON DOPED GAAS}

\subsection{Photoluminescence from unannealed GaAs:C}

Figure 4.1 shows the PL spectra of carbon doped GaAs epilayers at $77 \mathrm{~K}$ with hole concentrations (a) $4.3 \times 10^{19} \mathrm{~cm}^{-3}$. (b) $5.3 \times 10^{19} \mathrm{~cm}^{-3}$. (c) $1 \times 10^{-11} \mathrm{~cm}^{-3}$. From the peak position, peak $\mathrm{A}$ is considered as coming from the substrate emission which is not absorbed by epilayer. The substrate peak appears only from lowly doped materials. The stronger substrate peak in lower $p$ material is also attributed to the energy gap dependent absorption coefficient, $\alpha(E) \propto \sqrt{E-E_{k}}$. The $\mathrm{E}_{\mathrm{g}}$ decreases as carrier concentration increases as shown in Eq. 2.46. Therefore, $\alpha(1.508 \mathrm{eV})$ increases as carrier concentration increases, resulting in weaker substrate emission detected outside of the surface. Nevertheless, the intensity of peak A is much stronger compared to what is observed for about the same carrier concentrations from other studies $[76,78-81,122.123]$. This is due to very thin epilayer. $\sim 1000 \AA$.

With increasing hole concentration, the position of peak B is redshifted. The peak position is $1.452 \mathrm{eV}$ and $1.432 \mathrm{eV}$ for $p=4.3 \times 10^{19} \mathrm{~cm}^{-3}$ and $1 \times 10^{2(1)} \mathrm{cm}^{-3}$, respectively in Fig. 4.1. The dominant peak at low temperature due to band-to-band (B.B) or band-toacceptor $(\mathrm{e}, \mathrm{A})$ transition is the subject of controversy. As acceptor concentration increases up to $p \leq 1.4 \times 10^{19} \mathrm{~cm}^{-3}$ [124], the activation energy decreases to zero. In this situation. the transition via the acceptor level does not occur [125]. However. Nasledov' 't al. [126] proposed that the transition via the acceptor band was possible for the heary' 


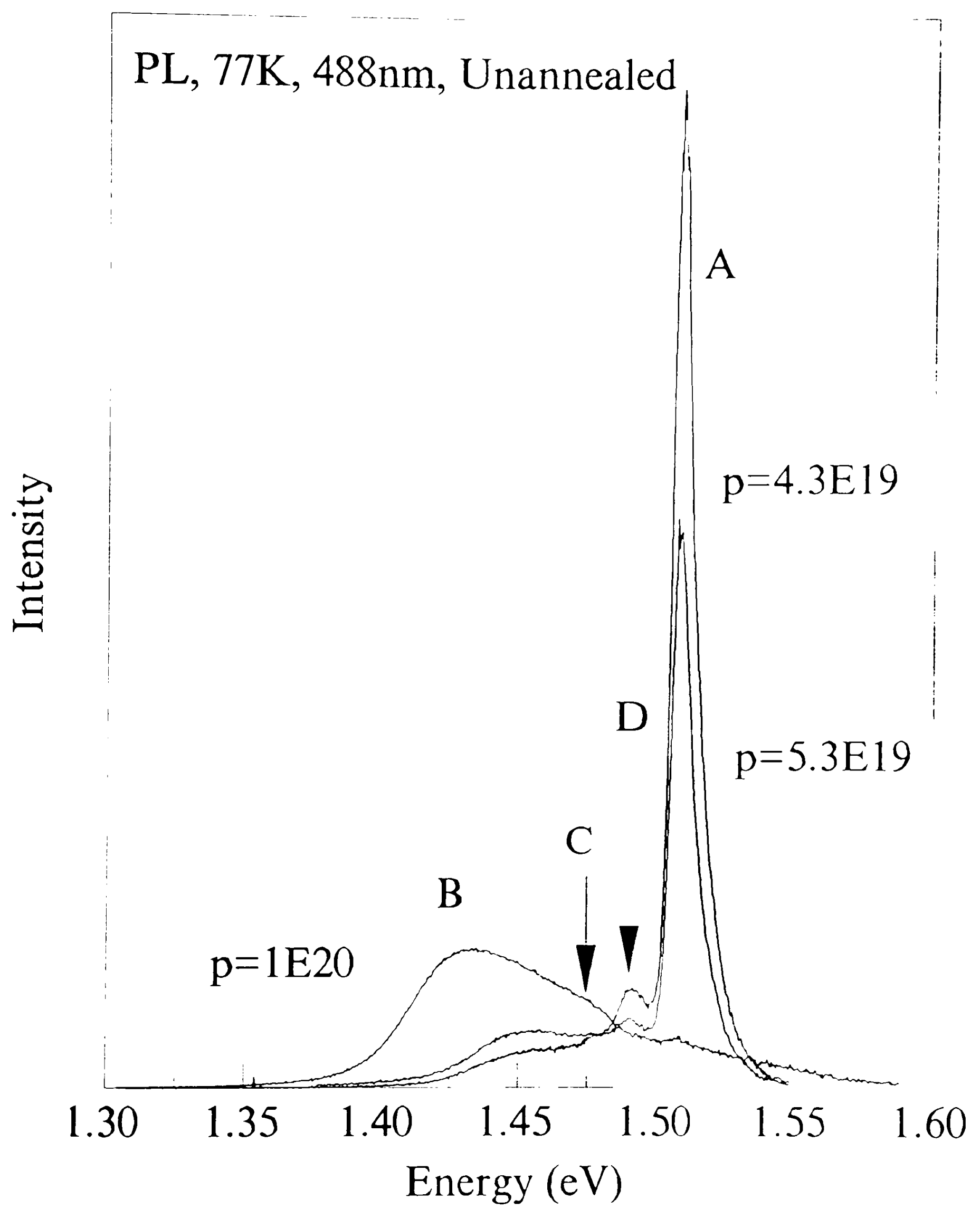

Figure 4.1: Photoluminescence spectra from unamnealed ( iats: (' samples for difterent carrier concentrations at $77 \mathrm{~K}$. $i_{心 \infty}=488 \mathrm{~nm}$. 
doping regime. The peak $\mathrm{B}$ might be coming from (e.A) transition. This identification agrees with that of Chen et al. [78] and Nathan et al. [94]. Nathan et al. made the identification from the PL peak energy study for varying concentration. PL peak of lowly Zn- [94] or C- [122] doped GaAs showed up at lower energy than that expected from Lic. 2.44 as shown in Fig. 4.2. The peak position of PL taken at $12 \mathrm{~K}$ from $p=1.9 \times 10^{16} \mathrm{~cm}^{-3}$ carbon doped GaAs was $\approx 1.494 \mathrm{eV}$, which is $\approx 25 \mathrm{meV}$ smaller than $\mathrm{E}_{\mathrm{g}}(12 \mathrm{~K}$ ) [80]. The difference is compatible to carbon impurity level in GaAs, which is $26 \mathrm{meV}$ [127]. The intensity of the (e.A) emission peak increased as the concentration increases. The redshift observed for peak B indicates the doping induced band gap shrinkage. The FWHM of peak B is increased as carrier concentration increases. The broadening might be caused by breakdown of the $\vec{k}$ - conserving process described in section $2.6[75,122]$. This broadening is accelerated by bandtail states and high energy side peak, C. and high energy tail at very high concentration. The peak of emission is plotted in Fig. 4.2.

The low-energy tail edge is found from the intersection between the tangent to the low energy tail of the emission band and the background [75]. The low-energy tail edge is regarded as the real band gap of the doped samples. They are 1.414, 1.413, and 1.390 $\mathrm{eV}$ for the $p=4.3,5.3$ and $10 \times 10^{19} \mathrm{~cm}^{-3}$ samples, respectively. in this study. This agrees well with what is expected from the following empirical equation [77].

$$
\Delta E_{g}=-2.6 \times 10^{-8} p^{1 / 3}
$$

The above equation describes the band gap shrinkage upon doping. This is due to pure band gap shrinkage, which can be described by a rigid shift of each band and shrinkage due to bandtail formation into the band gap, as shown in Figure 4.3. 


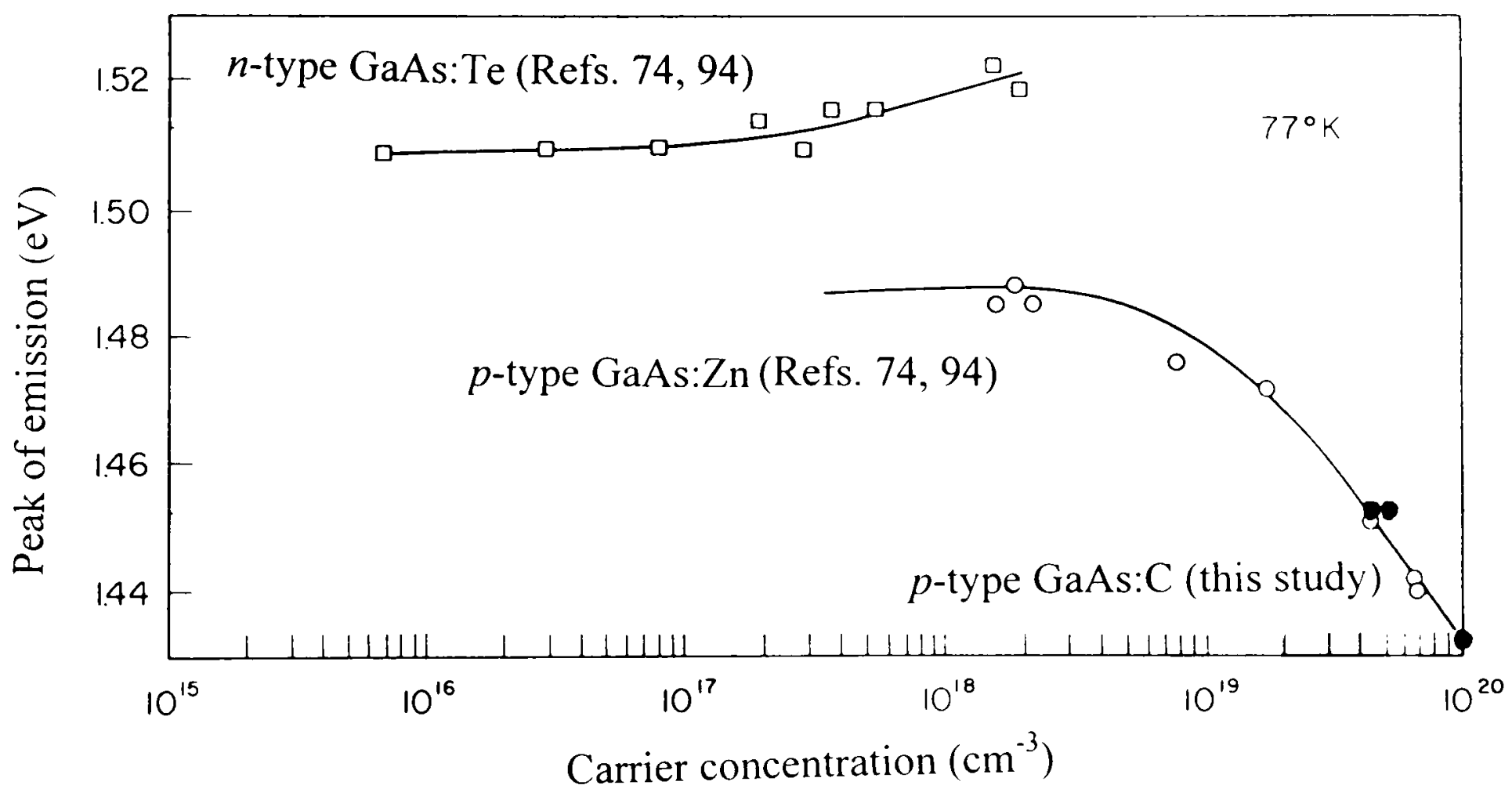

Figure 4.2: Position of the photoluminescence emission peak at $77 \mathrm{~K}$. 


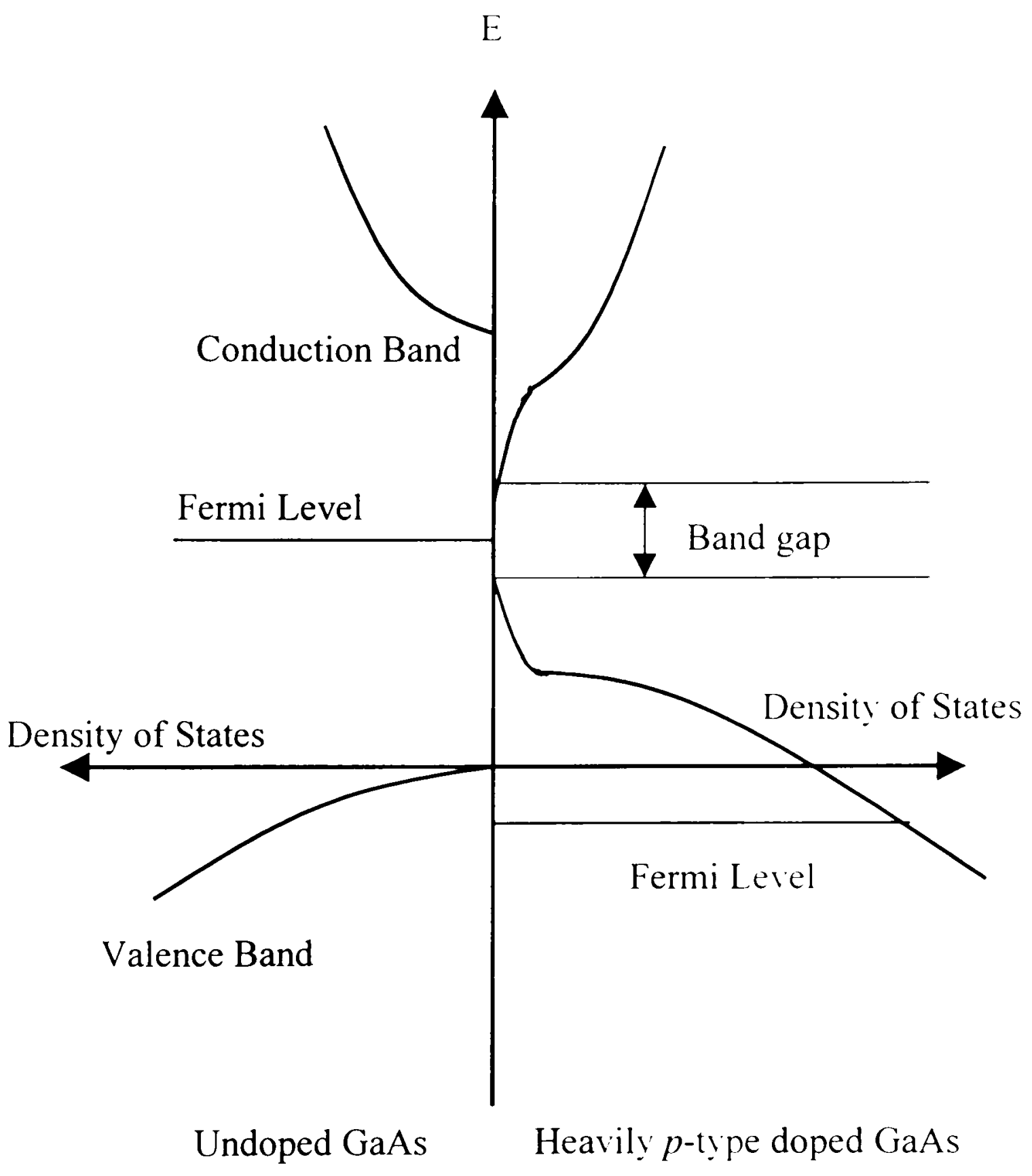

Figure 4.3: A schematic of the band structure in both undoped and heavily $p$-type doped GaAs [76]. 
A shoulder at the high energy side of the main PL peak in $p$-type GaAs has been observed [75, 77-81, 122.]. The identification of this peak is controversial. This peak is identified as coming from $E_{\underline{g}}+E_{F}[75,122]$. $E_{F}$ is the Fermi energy . Other identifications are conduction band-to-heavy hole band transition [80]. or conduction band-to-the bottom of impurity band of the epilayer [78], or from the substrate [27, 79. 81]. These discrepant identifications are mainly coming from concentration dependence of the highenergy peak position. The blue shift of the peak as concentration increases was observed by Olego et al. and Kim et al. [75. 122]. which is different from concentration independent peak position observed by Chen et al. . Lu et al.. and Lee et al. [78. 79. 81]. Because the high energy side peak appears only at very' high concentration range [78. 80], only the $p=1 \times 10^{20} \mathrm{~cm}^{-3}$ sample showed the peak. C. at $1.479 \mathrm{eV}$ in Fig. 4.1 . Therefore. on information on carrier concentration dependence of peak position is available. As a result. no clear identification on the peak can be made in this study.

The peak $\mathrm{D}$ shows up at $\approx 1.49 \mathrm{eV}$, which is $18 \mathrm{meV}$ lower that the energy of peak A. This peak appears when peak A shows up. Therefore, this peak is considered as coming from the substrate emission. From the peak position, peak D is considered to be coming from conduction band-to-acceptor impurity level transition unintentionally introduced to the substrate material during the growth processes.

The high energy tail develops at the highest concentration as shown in Fig. 4.1. This is coming from hot-electron PL [27]. At high doping lev'els, the Fermi level moves into the valence band and the upper lying valence-band states are filled with holes. Due to the large difference in effective masses between holes and electrons in GaAs, a small 
distribution of holes in the valence band can recombine with conduction band electrons. Consequently, the probability of recombination of hot electrons, i.e., those with energy greater than the conduction band minimum, is increased. Therefore, some of hot electrons contribute to luminescence before they are thermalized. resulting in a high energy tail.

\subsection{Photoluminescence from annealed GaAs:C}

Carbon doped GaAs samples are annealed at temperature $350{ }^{\circ} \mathrm{C} \sim 850^{\circ} \mathrm{C}$. The surface of samples annealed at low temperature does not show difference from that of unannealed samples. but, samples annealed at 750 , or $850{ }^{\circ} \mathrm{C}$ were covered with white powder which is considered as As related material. Black pits show up on the surface of the samples annealed at high temperature. To investigate the surface of unannealed and annealed samples. optical micrographs are taken as shown in Fig. 4.4. Fig. 4.4 (a) and (b) are. taken from unannealed $p=4.3 \times 10^{19} \mathrm{~cm}^{-3}$ and $p=1 \times 10^{20} \mathrm{~cm}^{-3}$ samples. respectively. Fig. 4.4 (c) and (d) are from annealed samples. There are black spots on the surface of unannealed samples, and the number of the spots is increasing as the carrier concentration increases. The number and size of them increases drastically upon annealing as shown in Fig. 4.4 (c) and (d).

The PL spectra taken from annealed $p=1 \times 10^{20} \mathrm{~cm}^{-3}$ sample are shown in Fig. 4.5. The PL spectra taken from $p=4.3 \times 10^{19} \mathrm{~cm}^{-3}$ and $p=5.3 \times 10^{19} \mathrm{~cm}^{-3}$ samples under $850^{\circ} \mathrm{C}, 1$ hour annealing are similar to those of $p=1 \times 10^{20} \mathrm{~cm}^{-3}$ sample following the same anneal. The intensity of peak B is drastically decreased. The strong decrease in PL intensity might be due to the hole concentration reduction [98] and the formation of nonradiative recombination centers $[98,100]$ induced by annealing. As mentioned in 


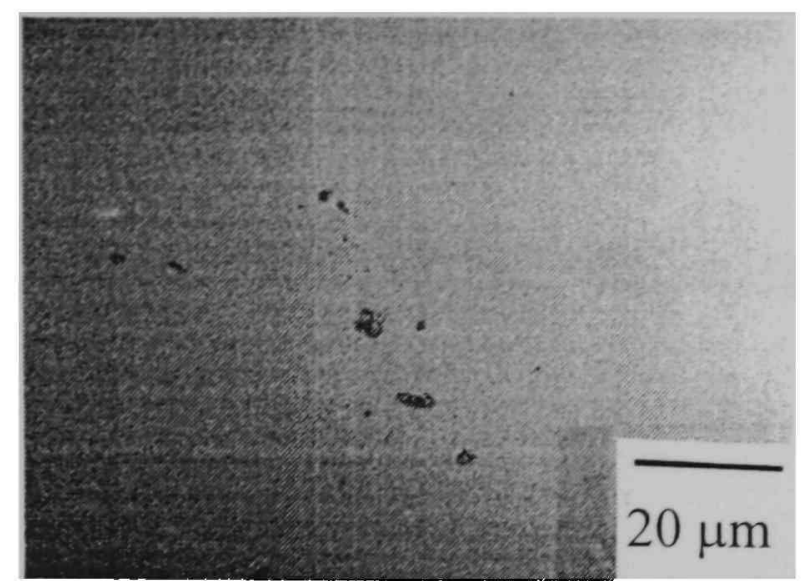

(a)

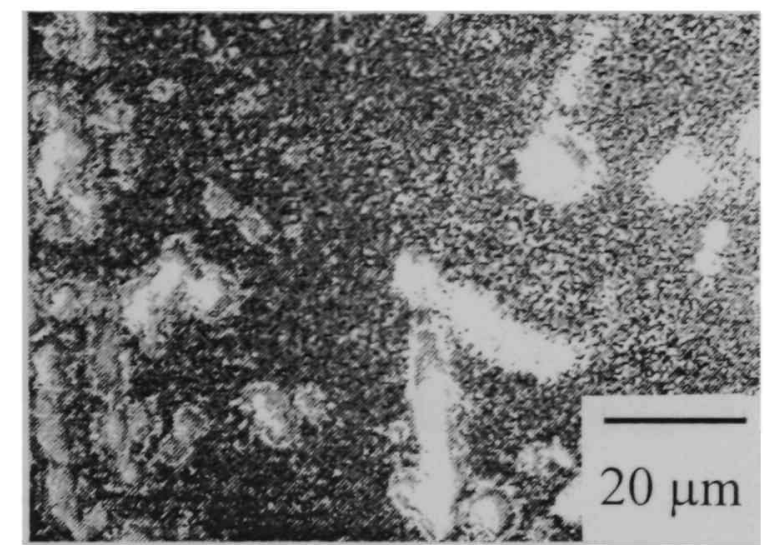

(c)

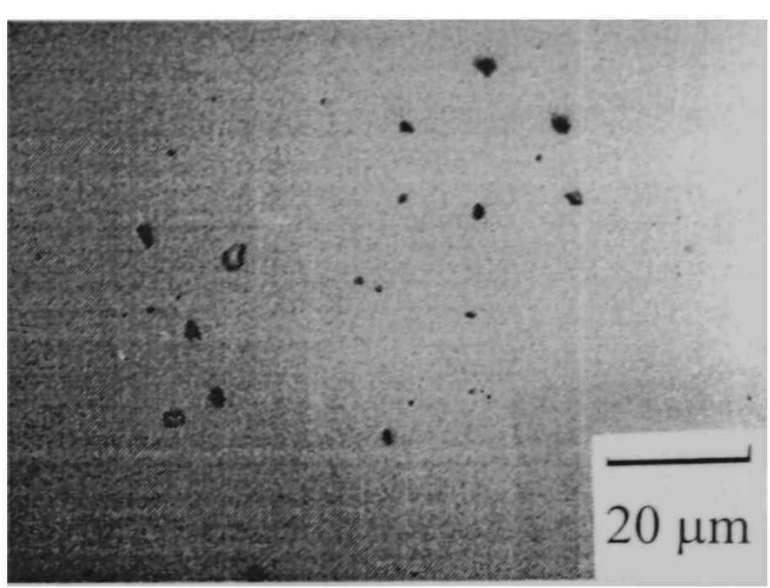

(b)

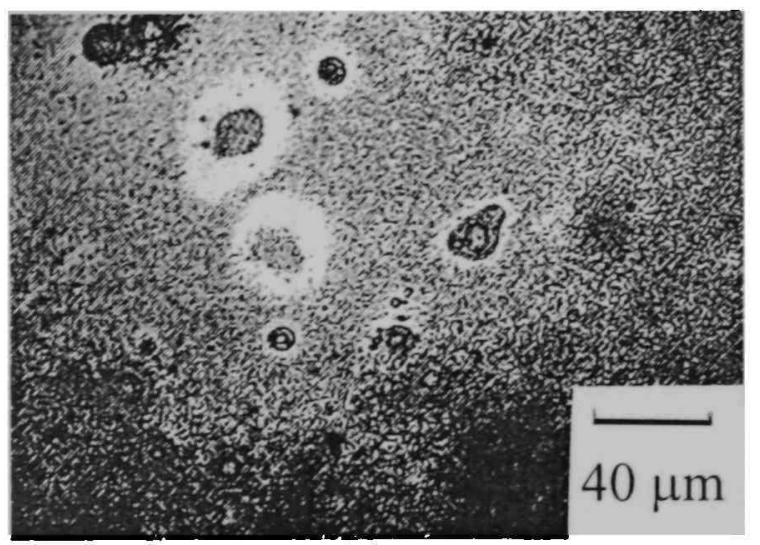

(d)

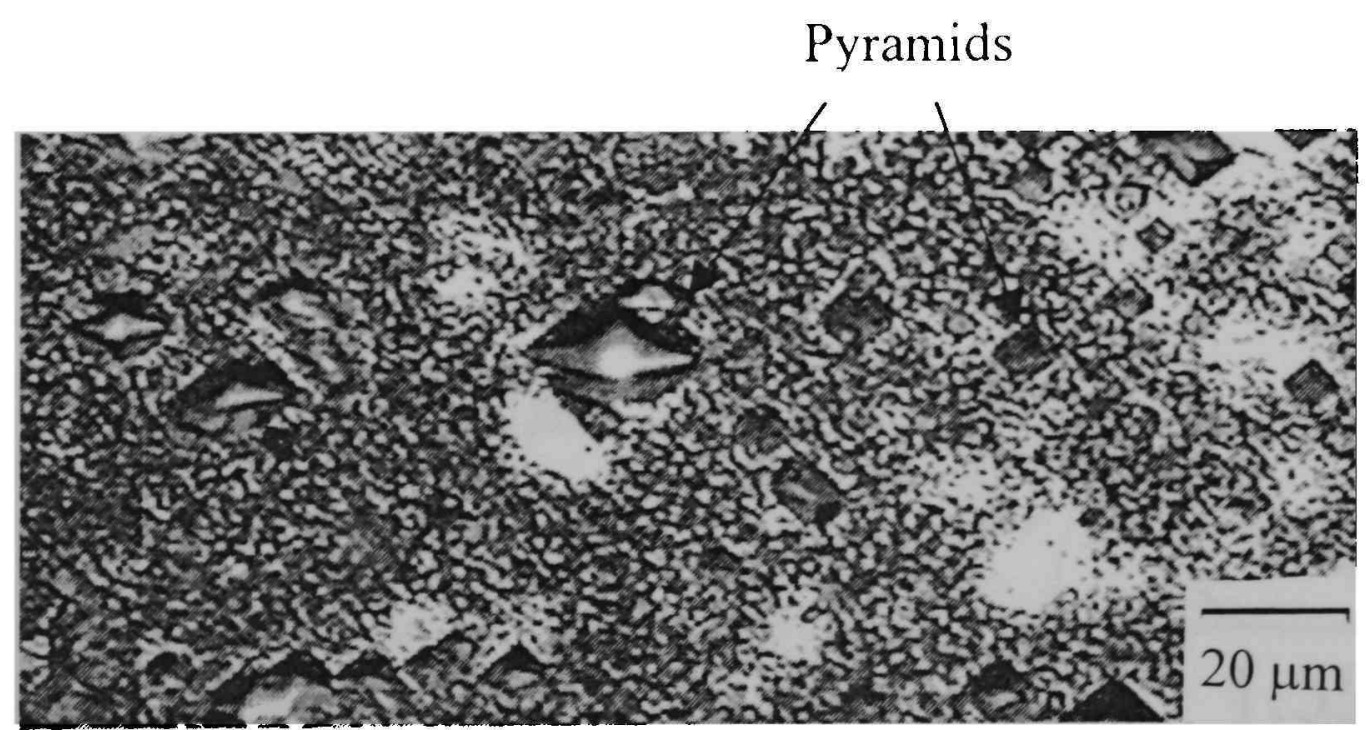

(e)

Figure 4.4: Optical-micrographs from unannealed (a) $p=4.3 \times 10^{19}$ $\mathrm{cm}^{-3}$ and (b) $p=1 \times 10^{20} \mathrm{~cm}^{-3}$ samples, and annealed (c) $p=4.3 \times 10^{19}$ $\mathrm{cm}^{-3}$, (d) $p=1 \times 10^{20} \mathrm{~cm}^{-3}$ samples. Theses are taken from capped area. Annealing conditions are $850^{\circ} \mathrm{C}, 1$ hour. (e) Taken from uncapped annealed $p=1 \times 10^{20} \mathrm{~cm}^{-3}$ sample. 


\section{$\mathrm{PL}, 77 \mathrm{~K}, 488 \mathrm{~nm}, \mathrm{p}=1 \times 10^{20} \mathrm{~cm}^{-3}$}

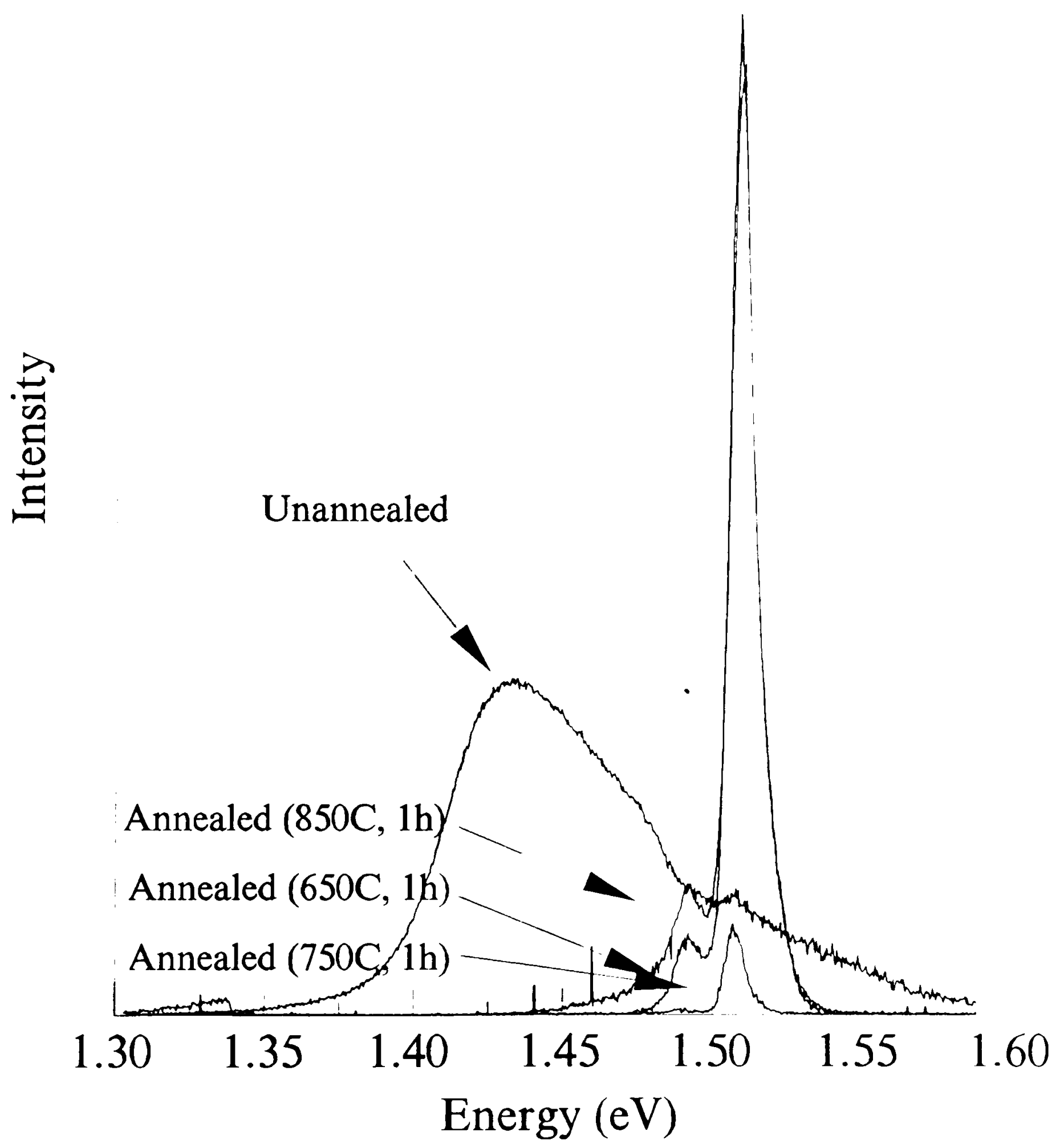

Figure 4.5: Photoluminescence spectra from annealed $p=1 \times 10^{2(1)} \mathrm{cm}^{-3}$ (iaAs: $\mathrm{C}$ sample: for different annealing conditions at $77 \mathrm{~K}$. í心 $=488 \mathrm{~nm}$. 
section 4.1 , the intensity of peak B is proportional to carrier concentration. The annealing causes hole concentration reduction, and this reduction might result in PL intensity decrease. The hole concentration reduction upon annealing will be discussed in more detail in Chapter V. The hole density reduction alone cannot explain the drastic decrease in PL intensity. The hole density reduction based on Raman spectra of unannealed and annealed $\left(850^{\circ} \mathrm{C}, 1\right.$ hour) $p=1 \times 10^{20} \mathrm{~cm}^{-3}$ sample in Fig. 5.13 is much smaller than that based on PL spectra in Fig. 4.1. The hole density estimation based on Raman spectra will be discussed in section 5.3. Therefore, the annealing might cause the formation of nonradiative recombination centers. Fushimi et al. [98] found the luminescence lifetime decrease with high temperature annealing, indicating more recombination centers. As one of the nonradiative recombination centers, misfit dislocation caused by strain relief was suggested [100]. However, the PL intensity reduction was not alleviated by reducing the layer thickness below the critical thickness, $h_{c}$, or adding indium to increase $h_{c}$ above the layer thickness [128]. This result suggests that the strain relief via misfit dislocation formation is not responsible for the PL intensity reduction upon annealing. One of compensation centers, $\mathrm{C}_{\mathrm{Ga}}$, is introduced to explain the hole concentration and $\mathrm{PL}$ intensity reduction. Watanabe et al. [129] observed peak around $1.148 \mathrm{eV}$ from unannealed and annealed carbon doped GaAs. The peak becomes more intense upon annealing. They proposed that this peak is related to $\mathrm{Ga}$ vacancy $+\mathrm{C}_{\mathrm{Ga}}$ donor center from its energy [130] and proposed the intensity of the peak as the index for the concentration of compensation centers such as $\mathrm{C}_{\mathrm{Ga}}$ donors. The peak was not observed from the samples studied in here. Therefore, based on their proposal, carbon compensation via $\mathrm{C}_{\mathrm{Ga}}$ is negligible in the annealed and unannealed samples studied in here. Carbon 
precipitation in annealed samples is observed from Raman spectroscopy study which is discussed in section 5.4. The radiation efficiency drops by an order of magnitude when the precipitates become detectable by transmission electron microscopy (TEM) for $n-t y p e$ GaAs doped with Se or Te with $n>3 \times 10^{18} \mathrm{~cm}^{-3}[9.131]$. Therefore, carbon precipitates are suggested for nonradiative recombination centers causing the PL intensity' reduction.

\subsection{Summary}

Photoluminescence spectra are studied for heavily carbon doped GaAs. Due to the energy dependent absorption coefficient and the thin epilayer thickness, emission from the substrate was observed. The main peak from the epilayer is attributed to band-toacceptor band transition. The peak is broadened and redshifted as carrier concentration increases. The broadening is attributed to non $\vec{k}$-conserving process. band tailing, and high energy tail at very high carrier concentration. The redshift is due to bandgap narrowing caused by doping. PL intensity from annealed samples is drastically decreased. Carrier concentration reduction and the formation of nonradiative recombination centers are suggested as the cause of the behavior. and carbon precipitates observed in Raman spectra are suggested as the possible nonradiative recombination centers. 


\section{CHAPTER V}

\section{RAMAN SPECTROSCOPY OF HEAVILY CARBON DOPED GAAS}

Raman spectra from heavily carbon doped GaAs are taken with three different excitation energies: $2.71 \mathrm{eV}, 2.54 \mathrm{eV}$, and $2.41 \mathrm{eV}$. Line shapes are found to be insensitive to photon energy over this range. The relationships between hole concentration $p$ and the intensity and energy shift of the LOPC mode are investigated. It is found that this mode, as well as the LO phonon, red shifts below the respective TOand LO-phonon energies in bulk GaAs. The $\mathrm{C}_{A}$ LVM intensity increases in direct proportion to $p$. This intensity measurement is compared with those of the more easily studied LOPC band.

\subsection{The $\mathrm{C}_{\mathrm{As}}$ local vibrational mode}

Raman spectra are shown in Figure 5.1 over the $C_{A s} L V M$ range for bulk GaAs and the $4.87 \times 10^{19} \mathrm{~cm}^{-3}, 7.54 \times 10^{19} \mathrm{~cm}^{-3}$ and $1 \times 10^{20} \mathrm{~cm}^{-3}$ samples. The LVM intensity increases with increasing $p$. These spectra are normalized with respect to 2 TO overtone intensity, and the ratio is plotted in Figure 5.2. The 2TO overtone intensity is used as an internal intensity standard for comparing spectra from sample to sample because it is proportional to the scattering volume, i.e., independent of $p$. This procedure eliminates experimental difficulties in measuring absolute scattering intensities.

Even though carbon is introduced as an acceptor in $p$-type GaAs. it may also incorporate as a substitutional donor or interstitial. This may cause inequality between 


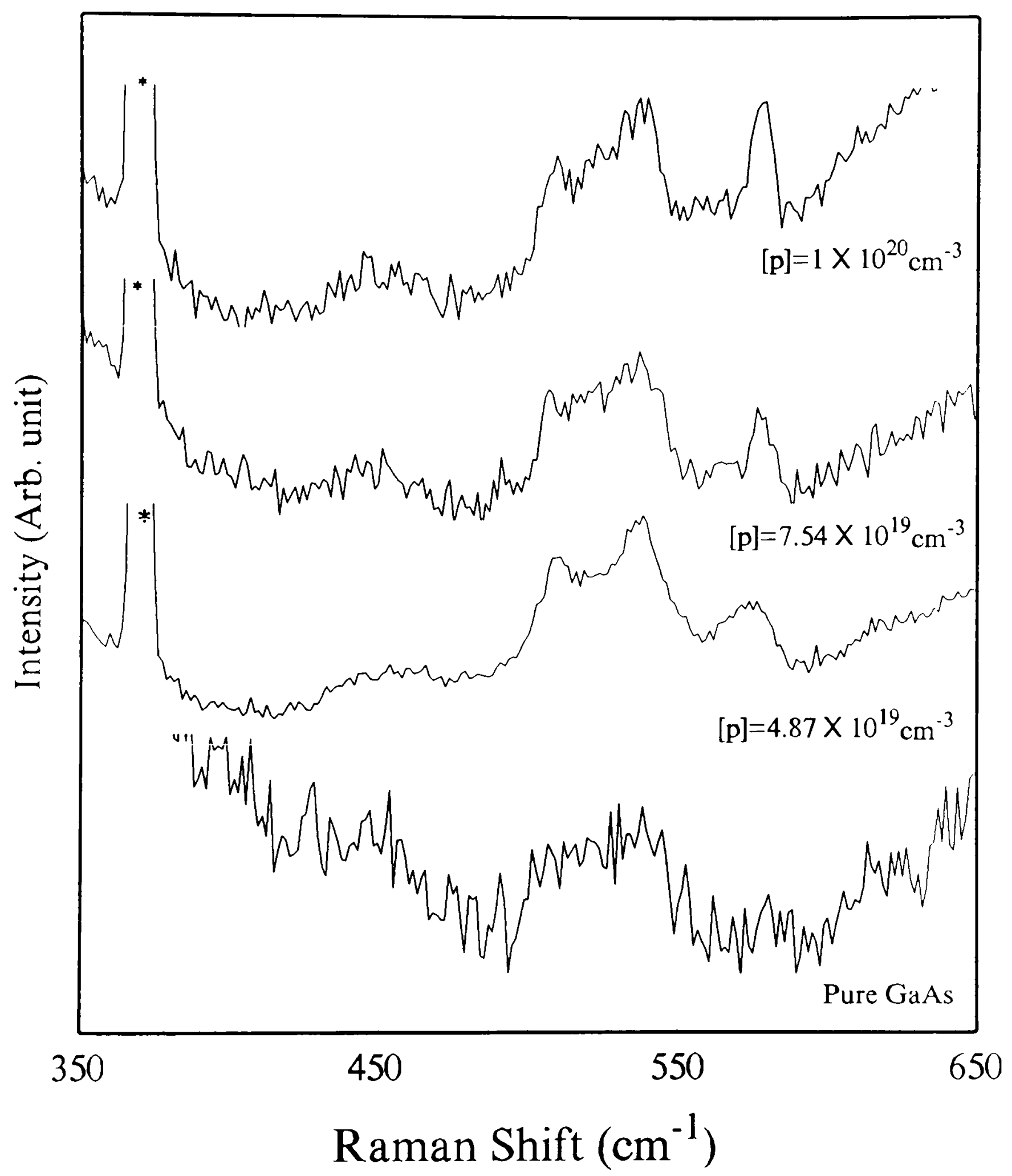

Figure 5.1: Room temperature Raman spectra in the $\mathrm{C}_{\mathrm{A}} \mathrm{LVM}$ range with $z(1,1) \bar{z}$ polarization configuration. $\lambda_{\mathrm{c} \backslash \mathrm{C}}=457.9 \mathrm{~nm}$. 


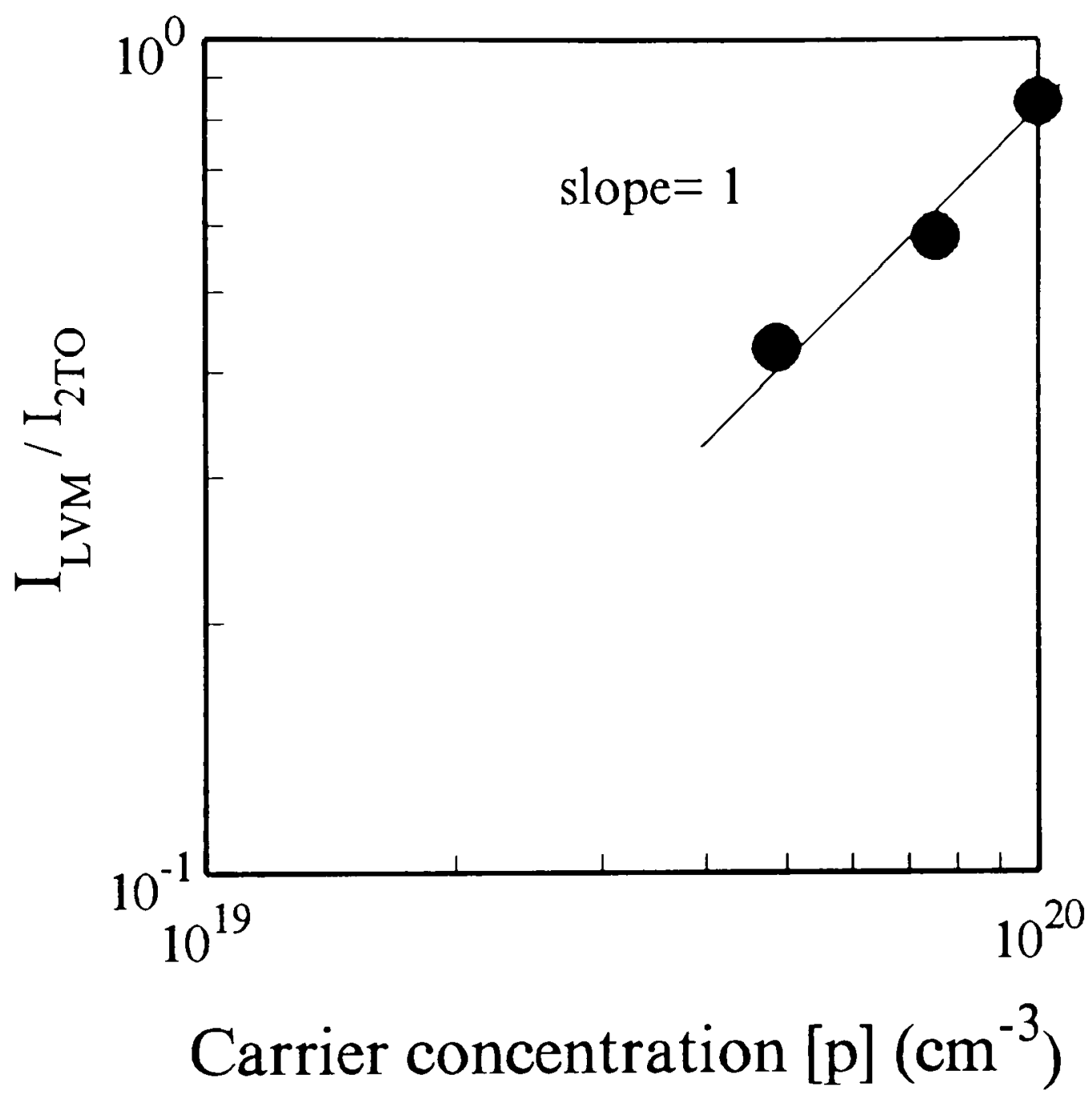

Figure 5.2: $\mathrm{C}_{\mathrm{As}} \mathrm{LVM}$ intensity normalized by $2 \mathrm{TO}$ overtone intensity in these samples studied. $\lambda_{\text {eic }}=457.9 \mathrm{~nm}$. 
carrier concentration and carbon at arsenic site concentration. The site distribution of carbon is determined by the surface stoichiometry, V:III flow ratio. laver growth temperature, and carbon and gallium source [132-134]. It is known that the carbon incorporation as an acceptor is higher at the lower V/III molar ratio and grouth temperature [103, 128, 133-135]. A wide range of carbon doping concentrations, with low compensation, is obtained in OMCVD growth of GaAs using $\mathrm{CCl}_{+}$[133]. Samples investigated in this study were grown at low V/III molar ratio and growth temperature by using $\mathrm{CCl}_{4}$ as a carbon source. Therefore, the self-compensation and interstitial concentrations should be small and the Hall concentrations close to $\mathrm{C}_{\mathrm{As}}$ concentration.

It is found from Figures 5.1 and 5.2 that in this set of samples, the relative LVM intensity $\mathrm{I}_{\mathrm{LVM}} / \mathrm{I}_{2 \mathrm{TO}}$ is directly proportional to the carrier concentration. This is in accord with Eq. 2.4 under the $p \approx\left[\mathrm{C}_{\mathrm{As}}\right]$ assumption made from the sample growth conditions. Since only the latter contributes to $\mathrm{I}_{\mathrm{LVM}}$, our data show the expected correlation between $\left[\mathrm{C}_{\mathrm{As}}\right]$ and the measured Raman intensity. Our results on OMCVD samples are more intuitive than those of Wagner et al. [39] mentioned in section 2.2 and validate the use of $\mathrm{I}_{\mathrm{LVM}} / \mathrm{I}_{2 \mathrm{TO}}$ to calibrate substitutional-site concentrations. Unfortunately, the LVM signals are weak and the superposition on $2 \mathrm{LO}(\Gamma)$ limit their practical use for determining $\left[\mathrm{C}_{\mathrm{As}}\right]$. Therefore. any effects which cause a deviation between $p$ and $\left[\mathrm{C}_{\mathrm{As}}\right]$ would not be observed with intensity vs. $p$ we report.

Interestingly, we find that the LO phonon also serves as a good internal intensity standard. This conclusion is reached by examining the $C_{A s} L V M$ intensity. normalized to $\mathrm{I}_{\mathrm{LO}}$. The normalized LVM intensity with respect to that of LO phonon are shown in Figure 5.3 (gray squares). We find direct dependence of $I_{L V M I}$ on $p$, as when the TO 


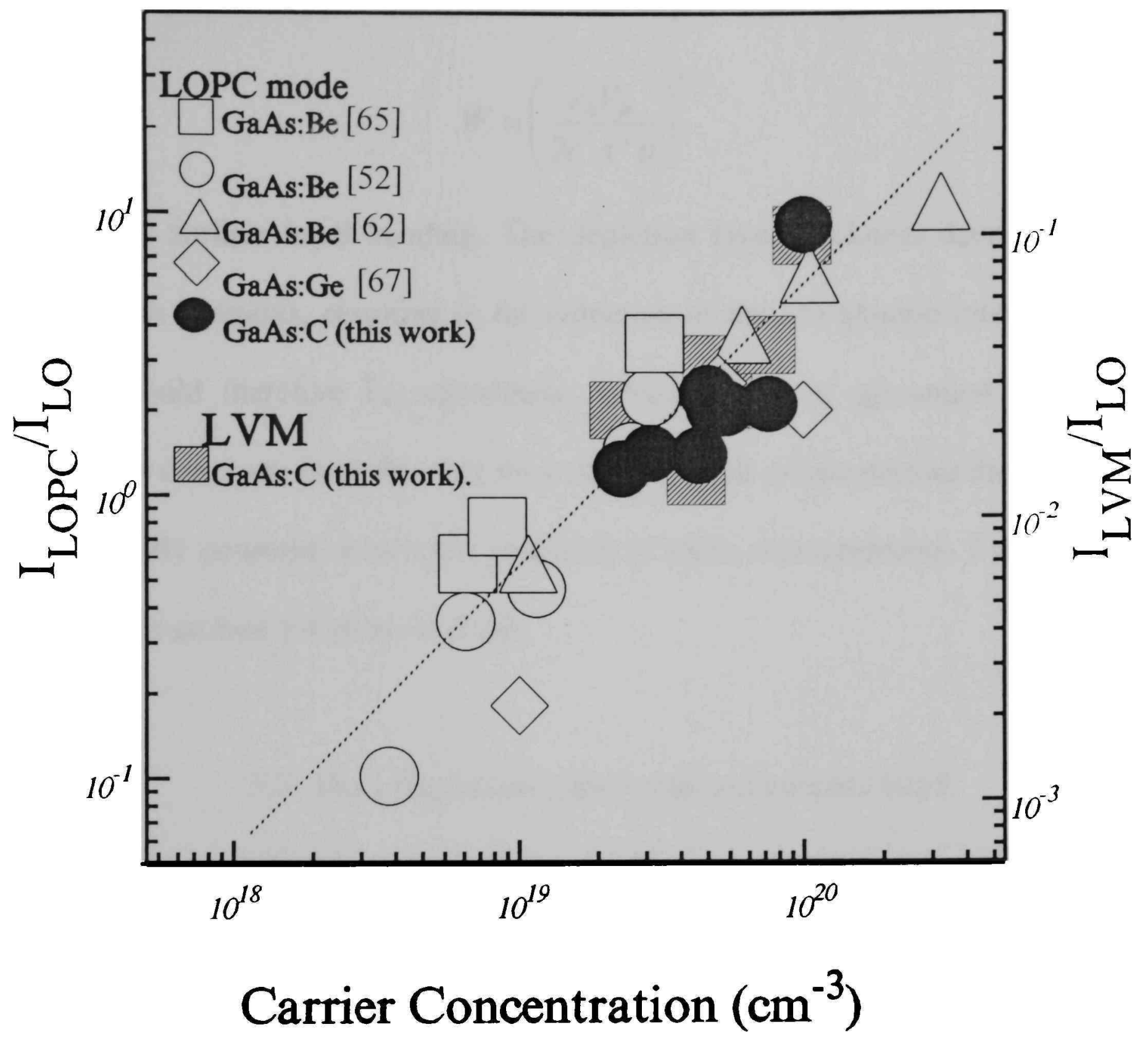

Figure 5.3: Compilation of relative intensities of the $\mathrm{C}_{\mathrm{As}}$ LVM (gray squares, right-hand scale) and the LOPC band (filled circles. left-hand scale) to the LO mode from this study. Other data are for GaAs doped with Be from Refs. 52, 62, 65 and Ge, Ref. 67. The dashed line corresponds to direct dependence between the relative intensities and the carrier concentration. 
phonon overtone intensity was used as the internal reference. This is unexpected. since the "bare" LO phonon stems from the near-surface hole-depletion region. The thickness of the hole-depletion layer. W, should decrease as $p$ increases [136]:

$$
W=\left(\frac{\varepsilon_{0} I_{B}}{2 e^{2} \pi^{2} p}\right)^{1 / 2}
$$

where $V_{B}$ is surface band bending. The depletion layer thickness decreases as carrier concentration increases, resulting in the reduction of the LO phonon intensity. The ratio $\mathrm{I}_{\mathrm{LVM}} / \mathrm{I}_{\mathrm{LO}}$ should therefore be superlinear, which is not in agreement with what we observe. We conclude from this that for extremely high $p$-type doping the hole-depletion width is nearly constant, within the precision of these measurements. I will discuss this conclusion in section 5.4 in more detail.

\subsection{The Longitudinal optic-plasmon coupled band}

Raman spectra are shown in Figure 5.4 for varying carbon concentrations over the optic phonon energy range in the $(\mathrm{x}, \mathrm{y})$ polarization configuration with $2.71 \mathrm{eV}$ of excitation energy. The positions of two vertical lines correspond to energies of $\mathrm{TO}$ and LO phonon in bulk GaAs. Two main peaks are observed in each spectrum. The bands exhibit the polarization properties of the LO phonon, that is, allowed in $(x, y)$ and $\left(x^{\prime} . x^{\prime}\right)$, and forbidden in $(x, x)$ and $\left(x^{\prime}, y^{\prime}\right)$ conditions. This will be discussed in more detail in Figure 5.5. The peak near $270 \mathrm{~cm}^{-1}$ is identified as the LOPC band. This identification is based on the peak position, polarization selection rules. and intensity trends. as discussed later. The second band near $292 \mathrm{~cm}^{-1}$ is the GaAs LO phonon mode coming primarily from the near-surface, hole-depletion region $[62,65.137]$. Both bands are symmetric. 


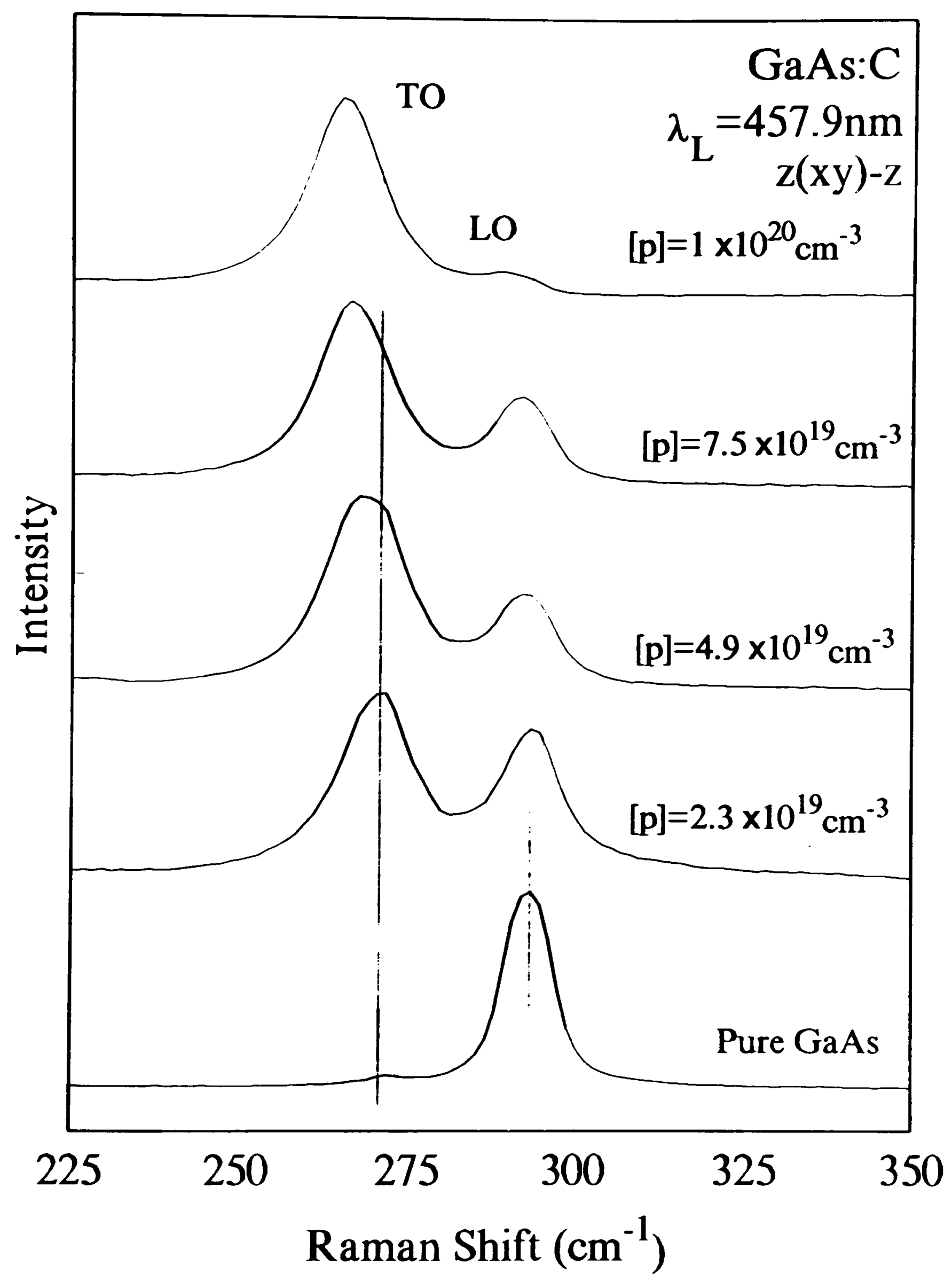

Figure 5.4: Room-temperature, symmetry allowed Raman spectra of carbon doped GaAs samples studied here, spanning the range of hole concentrations studied. Data are normalized with respect to LOPC band intensity. A systematic change in relative intensities and red shifting are observed. 
(a) $\quad p=2.3 \times 10^{19} \mathrm{~cm}^{-3}$
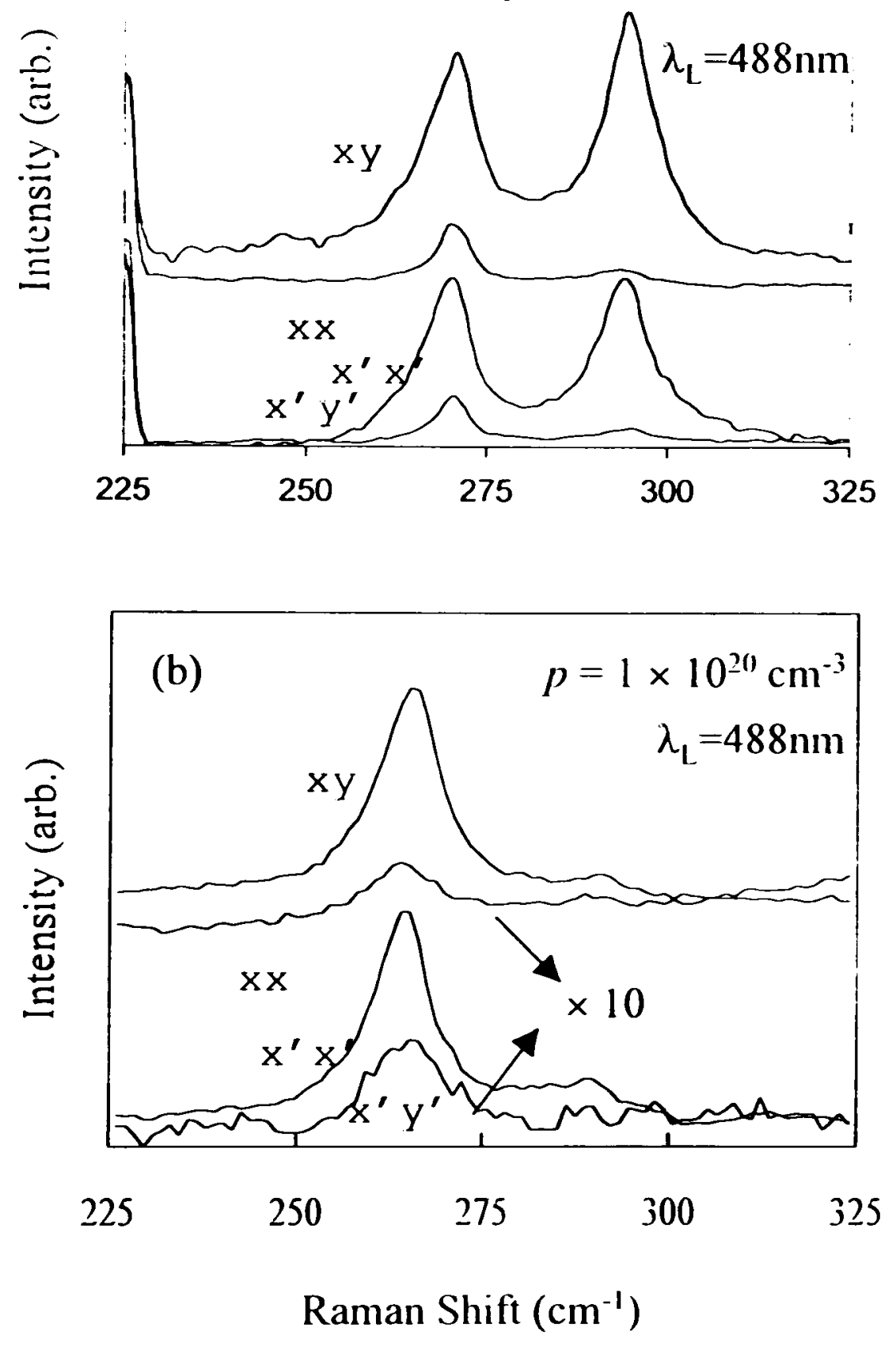

Figure 5.5: Polarized Raman spectra of the (a) most lightly and (b) most heavily doped samples studied here. Line shapes and positions in (b) do not vary significantly between the different spectra. which is different in (a). 
and fit well by Lorentzian line shapes with widths ranging between 8 and $10 \mathrm{~cm}^{-1}$. Thesc shapes vary little across the full range of $p$ studied, showing no significant asymmetry' as is characteristic of the LOPC at lower carrier concentrations [51, 59.65.67].

The intensity of the LOPC mode exceeds that of the LO phonon band in each spectrum shown (high $p$ ). This is in contrast to the Raman spectrum from undoped GaAs for backscattering along this crystal axis, which is dominated by the LO phonon at 292 $\mathrm{cm}^{-1}$, and exhibits only weak forbidden scattering from the TO line near $268 \mathrm{~cm}^{-1}$ due to experimental factors [138]. Two trends are clear in Fig. 5.4. First. the LOPC band intensity grows with increasing doping. As we discuss later, this is in direct proportion to the carrier concentration. The second trend in Fig. 5.4 is that both bands red shift at extremely high $p$. Over the range of $p$ studied here. the LOPC and LO lines shift by $-6 \pm$ $1 \mathrm{~cm}^{-1}$ and $-5 \pm 1 \mathrm{~cm}^{-1}$. respectively, below the TO-phonon $\left(268 \mathrm{~cm}^{-1}\right)$ and LO-phonon $\left(292 \mathrm{~cm}^{-1}\right)$ energies in undoped GaAs. Red shifts of the LOPC band have also been reported in GaAs doped with $\mathrm{Zn}[51,66]$, Be [52], and Ge [67].

To better understand the phonons in GaAs:C. we also examined forbidden scattering across the range of carrier concentrations in Fig. 5.4. Figure 5.5 shows spectra for the $2.3 \times 10^{19} \mathrm{~cm}^{-3}$ and $1 \times 10^{20} \mathrm{~cm}^{-3}$ samples in both allowed and forbidden scattering, obtained using $488.0 \mathrm{~nm}$ excitation. Spectra for the allowed $(x . y)$ and $\left(x^{\prime} . x^{\prime}\right)$ conditions were the same for each $p$. Likewise for the $(x . x)$ and $\left(x^{\prime} \cdot y^{\prime}\right)$ forbidden polarization conditions. The forbidden LO phonon was not observed in Fig. 5.5 . Therefore, the surface field effect is considered negligible according to Table 2.3. Since the band we measure near $270 \mathrm{~cm}^{-1}$ exhibits the polarization properties of the LO phonon. we believe it to be the LOPC band rather than TO phonon scattering. Enhanced scattering 
by TO phonons (e.g.. when due to the presence of impurities in high concentrations). which are forbidden in backscattering along the (001) axis, should be seen in all polarizations. Under these circumstances, we would also expect broadening of the T() and LO bands. The symmetric lineshape observed in this carrier concentration range is consistent with experimentally and theoretically established trends in p-type Gats 151. 59. 60, 62]. At lower $p$ in Fig. 5.5 (a), the forbidden "LOPC" mode is slightly narrower than in the allowed configuration, and the two lines are closer in intensity. This band. when observed in forbidden scattering, is most likely a superposition of the TO phonon and the LOPC band. At higher $p$, as shown in Fig. 5.5 (b). the forbidden LOPC band dominates in all polarizations, since the band positions and shapes are identical. The experimental equivalence between spectra taken in the $(x, y)$ and $\left(x^{\prime} \cdot x^{\prime}\right)$ and in the $(x, x)$ and $\left(x^{\prime}, y^{\prime}\right)$ configurations agrees with what is reported by Irmer ct al. [51]. In backscattering along the (001) surface with $\left(x^{\prime}, x^{\prime}\right)$ configuration. the deformation potential (DP). electro-optic and charge density fluctuation contribute to the LOPC intensity. while with $(\mathrm{x}, \mathrm{y})$ polarization only DP and electro-optic mechanisms contribute [51]. Irmer et al. could not measure any remarkable difference in $\left(x^{\prime}, x^{\prime}\right)$ and $(x . y)$ spectra and conclude from this result that DP and electro-optic scattering dominate over the charge density mechanism in this carrier concentration range. Peak energies in allowed and forbidden scattering geometries red shift along the same trend established in Fig. 5.4 with $457.9 \mathrm{~nm}$ excitation. Our measurements do not permit us to conclusively determine the TO phonon energy in our samples. 
The Stokes Raman scattering rate by LOPC mode, $I_{s}(\omega)$, can be written in the long-wavelength limit by taking into account both allowed deformation potential and electro-optic contribution as [62]:

$$
I_{s}(\omega) \propto\left(n_{\omega}+1\right) \frac{\left[\omega_{T o}{ }^{2}(1+C)-\omega^{2}\right]^{2}}{\left(\omega_{T o}{ }^{2}-\omega^{2}\right)^{2}} \operatorname{Im}\left(\frac{-1}{\varepsilon(\omega)}\right)
$$

where $\varepsilon(\omega)$ is the total dielectric function of the coupled system given by Eq. 2.24. $n_{(0)}$ is the Bose-Einstein function as follows:

$$
n_{\omega}=\frac{1}{e^{\frac{\hbar \omega}{k_{k} T}}-1}
$$

where $k_{\mathrm{B}}$ is Boltzmann constant. $\mathrm{C}$ in Eq. 5.2 is the Faust-Henry coefficient. This coefficient determines the relative magnitude of the atomic displacement and electrooptic contributions $[69,139]$. The value of the Faust-Henry coefficient may be obtained from data on the relative intensity of Raman scattering by polaritons, TO phonons and LO phonons $[54,140,141]$. It can be written down as follows [68]:

$$
C=\frac{e^{*} \hat{\varepsilon}_{l,} \cdot(\partial \chi / \partial u) \cdot \hat{\varepsilon}_{s}}{\mu \omega_{T o}{ }^{2} \hat{\varepsilon}_{l .} \cdot(\partial \chi / \partial E) \cdot \hat{\varepsilon}_{S}}
$$

In Eq. 5.4, $e^{*}$ and $\mu$ are the dynamical charge and the reduced mass of the ions, respectively. The tensor $(\partial \chi / \partial u)$ describes light scattering by the deformation potential mechanism, and $(\partial \chi / \partial E)$ electro-optic contribution. The value of the Faust-Henry coefficient $\mathrm{C}$, for $\lambda_{\text {exc }}=488.0 \mathrm{~nm}$ and $\mathrm{T}=300 \mathrm{~K}$ has been taken $\mathrm{C}=-0.57$ [138]. The $\operatorname{Im}\{-1 / \varepsilon(\omega)\}$ term can be expressed as follows: 


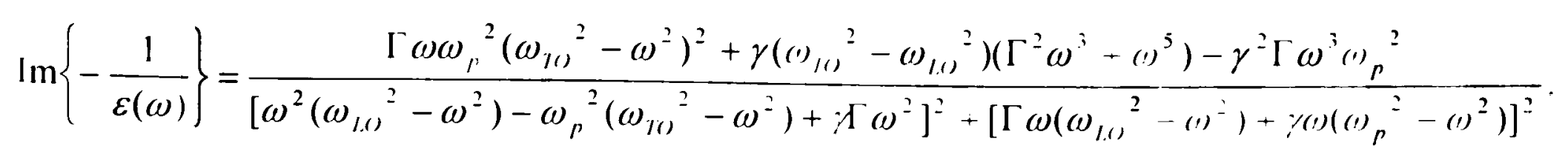

By substituting $\operatorname{Im}\{-1 / \varepsilon(\omega)\}$ into Eq. 5.2. $I_{5}(\omega)$ takes the following form

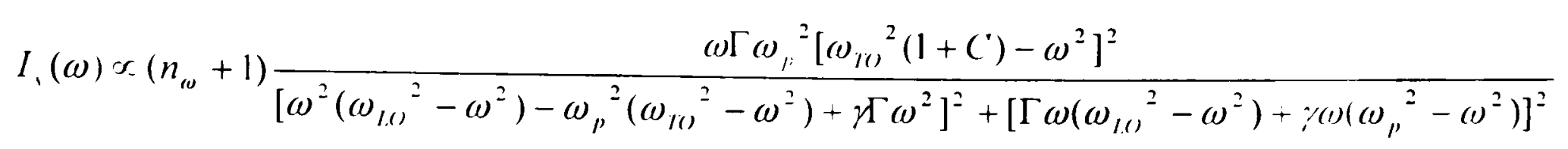

In this relation, $\gamma$-dependent terms in the numerator are neglected because the phonon damping. $\gamma$, is comparatively smaller than the plasmon damping $\Gamma$.

Figure 5.6 represents the calculated $I_{s}(\omega)$. The parameters used in the calculation are shown in Table 5.1. Normalized Raman rates are grouped with the same plasmon damping constant, $\Gamma$. At small $\Gamma$, two LOPC modes are clearly visible, and their behaviors as a function of plasmon frequency coincide with what is described in Fig. 2.5. At high $\Gamma$, only one LOPC mode is detected. Its FWHM and asymmetry decrease as $\omega_{\mathrm{p}}$ $\left(>500 \mathrm{~cm}^{-1}\right)$ increases.

Figure 5.7 represents the calculated $I_{(}(())$which is grouped with the same plasmon frequency. At low plasmon frequency. the shape and peak position of higher branch LOPC modes do not change as $\Gamma$ increases. This trend is changed for $\approx \omega_{\mathrm{p}}$. The shape and peak position of LOPC modes change drastically. And this trend is reversed again in higher plasmon frequencies. At $\omega_{\mathrm{p}}=1500 \mathrm{~cm}^{-1}$. the LOPC mode shows up near $\omega_{\mathrm{TO}}$, and its FWHM increases slightly as $\omega_{\mathrm{p}}$ increases.

At carrier concentration $\leq 2 \times 10^{19} \mathrm{~cm}^{-3}$ published lineshapes vary drastically. The lineshape of the LOPC band has been interpreted using the DP and Fröhlich electronphonon interaction mechanisms $[51,59,62.64,144]$. In our measurements for very high 


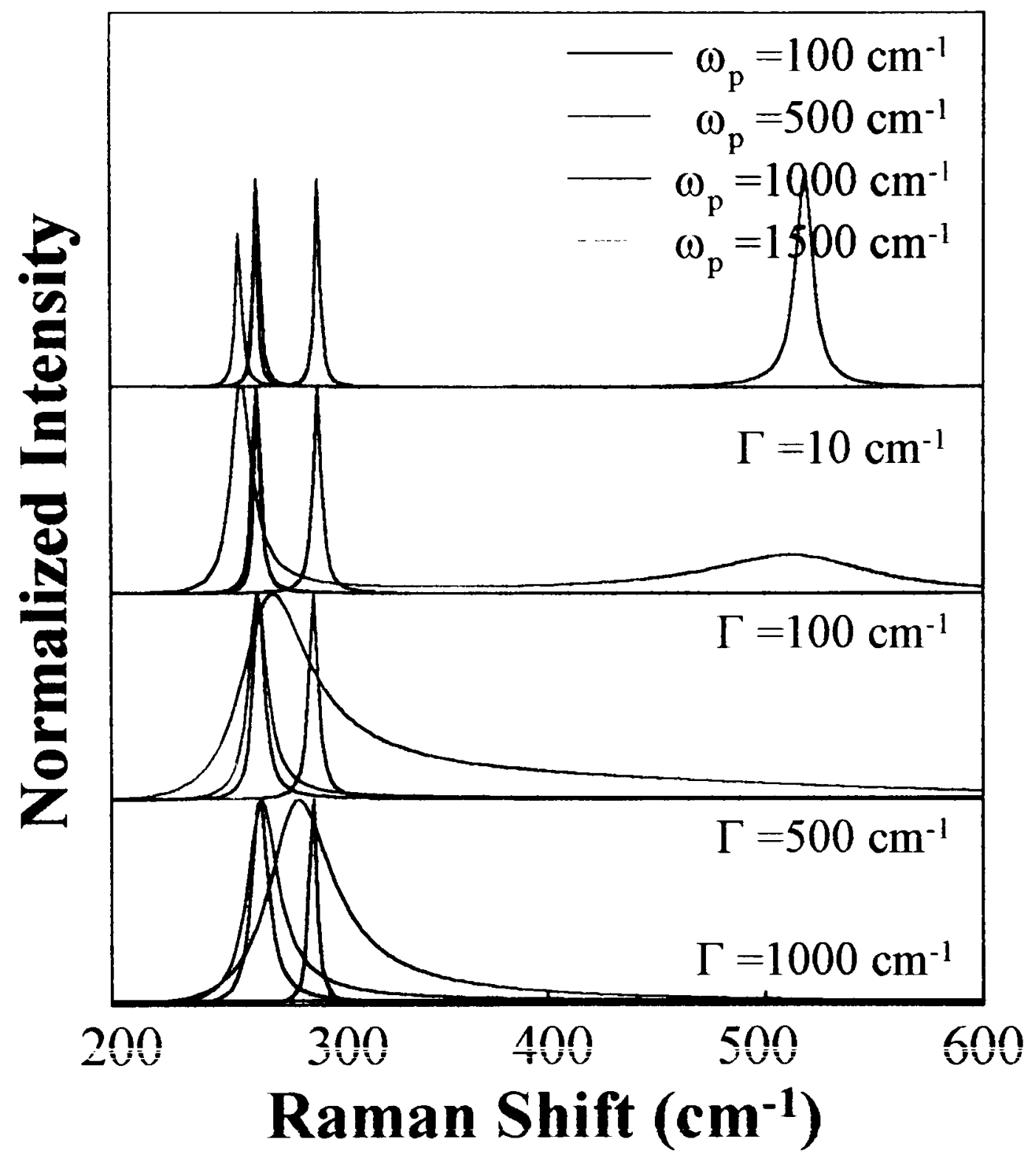

Figure 5.6: The Raman scattering rate calculated by Eq. 5.6. Spectra are grouped by the same plasmon damping constant. 
Table 5.1: List of parameters used for Eq. 2.24 and Eq. 5.6.

\begin{tabular}{lcc}
\hline Simbol & Description & Value \\
\hline$\varepsilon_{\infty}$ & $\begin{array}{c}\text { High-frequency } \\
\text { dielectric constant } \\
\text { Heavy-hole effective } \\
\mathrm{m}_{\mathrm{hh}}{ }^{*}\end{array}$ & $10.89^{\mathrm{a}}$ \\
$\mathrm{m}_{\mathrm{hh}}{ }^{*}$ & $\begin{array}{c}\text { mass } \\
\text { Light-hole effective } \\
\text { mass }\end{array}$ & $0.50 \mathrm{~m}_{0}^{\mathrm{a}}$ \\
$\gamma$ & $\begin{array}{c}\text { Phonon damping } \\
\text { constant }\end{array}$ & $0.076 \mathrm{~m}_{0}^{\mathrm{a}}$ \\
$\omega_{\mathrm{LO}}$ & $\begin{array}{c}\text { LO-phonon frequency } \\
\omega_{\mathrm{TO}}\end{array}$ & $2.5 \mathrm{~cm}^{-1 \mathrm{~b}}$ \\
$\mathrm{~T}$ & $\begin{array}{c}\text { TO-phonon frequency } \\
\text { Temperature }\end{array}$ & $292 \mathrm{~cm}^{-1}$ \\
\hline
\end{tabular}

${ }^{\mathrm{a}}$ Reference 142.

${ }^{b}$ Reference 143. 


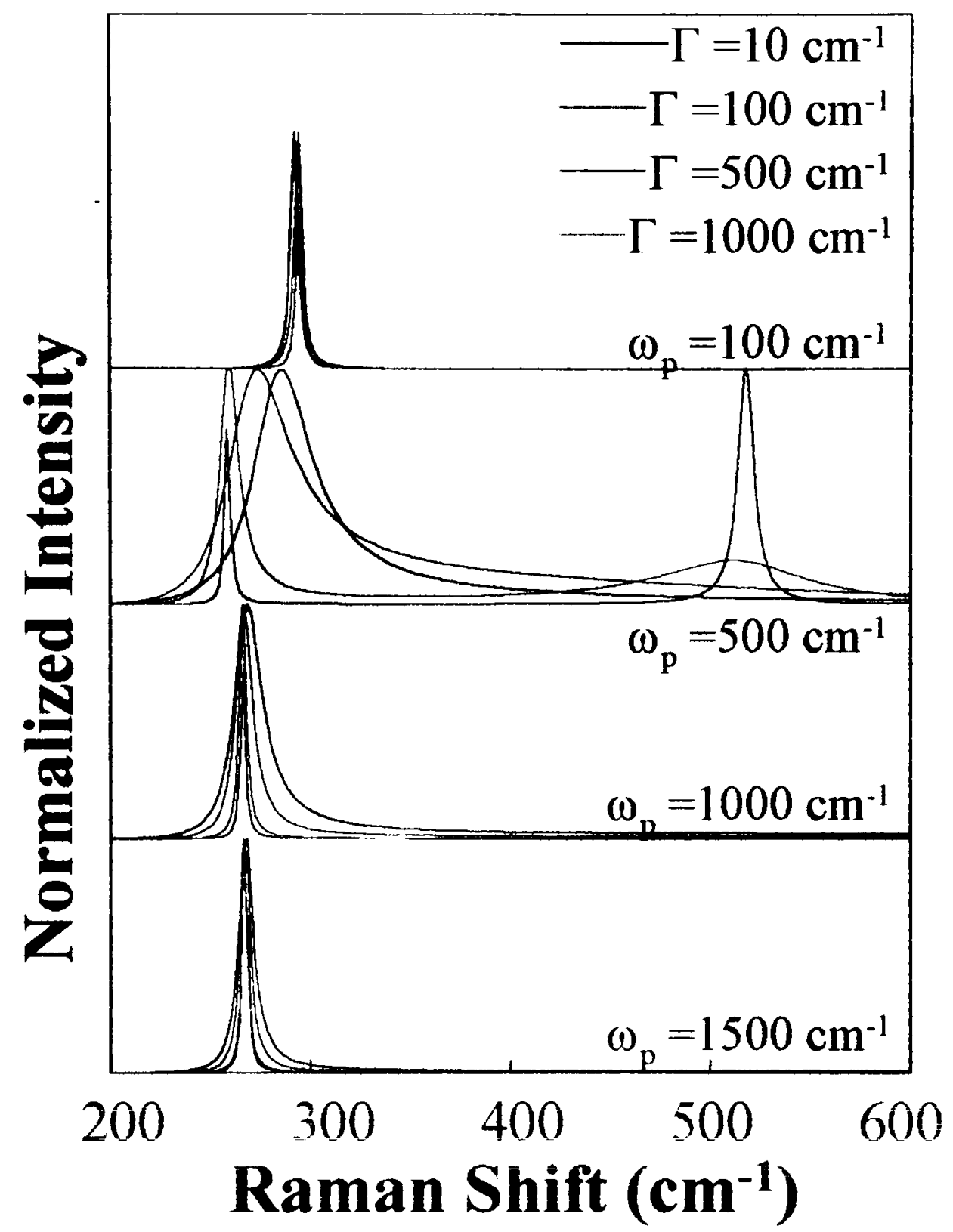

Figure 5.7: The Raman scattering rate calculated by Eq. 5.6. Spectra are grouped by the same plasmon frequency. 
carrier concentrations the LOPC lineshape shows no systematic variation 1 ith carrier concentration as shown in Fig. 5.4. This is consistent with what is observed and expected based on other studies $[51,62,66,145]$. This behavior makes no systematic information to be drawn from the lineshape analysis. Therefore, we focus our attention on the LOPC mode energy which shows a systematic shift in our experiments. Figure 5.8 summarizes changes in the peak positions of the LO phonon, which is believed to come from the depletion region, and the LOPC band versus carrier concentration. The solid squares are our data. Included in Fig. 5.8 (a) is the result from GaAs:Be [1+5] for the I.O mode and in Fig. 5.8 (b) are the results from GaAs:Zn [51] and GaAs:Be [52] for the LOPC mode. The solid line is a fit to my data in Fig. 5.8 (a). The redshift for our samples agrees with what has been established for Be doped GaAs [52] but is larger than that of Irmer et al. for $\mathrm{Zn}$ doped GaAs [51]. Interestingly, both the carbon and beryllium doped GaAs show stronger red shifting in both data sets in Fig. 5.8 (a) and (b). The data in Fig. 5.8 (a) suggest that it shifts more for small atom substitutional doping than in the case of GaAs doped with $\mathrm{Zn}$ which is in the same row of the periodic table as Ga and As. I suggest below that LO phonon red shift must be taken into account in treating the LOPC energy.

The LOPC mode red shift is explained by considering the dielectric function of $p$ type GaAs, $\varepsilon(\omega)$ [51, 52]. The dielectric function is well described by the sum of a Lorentzian form for phonons and a Drude form for free carriers as shown in Eq. 2.24 [52$54,57,58,60-64]$. The plasma frequency. $\omega_{p}$, in Eq. 2.24 is defined as

$$
\omega_{n}{ }^{2}=\frac{4 \pi p c^{2}}{\varepsilon_{s} m_{h}^{*}}
$$



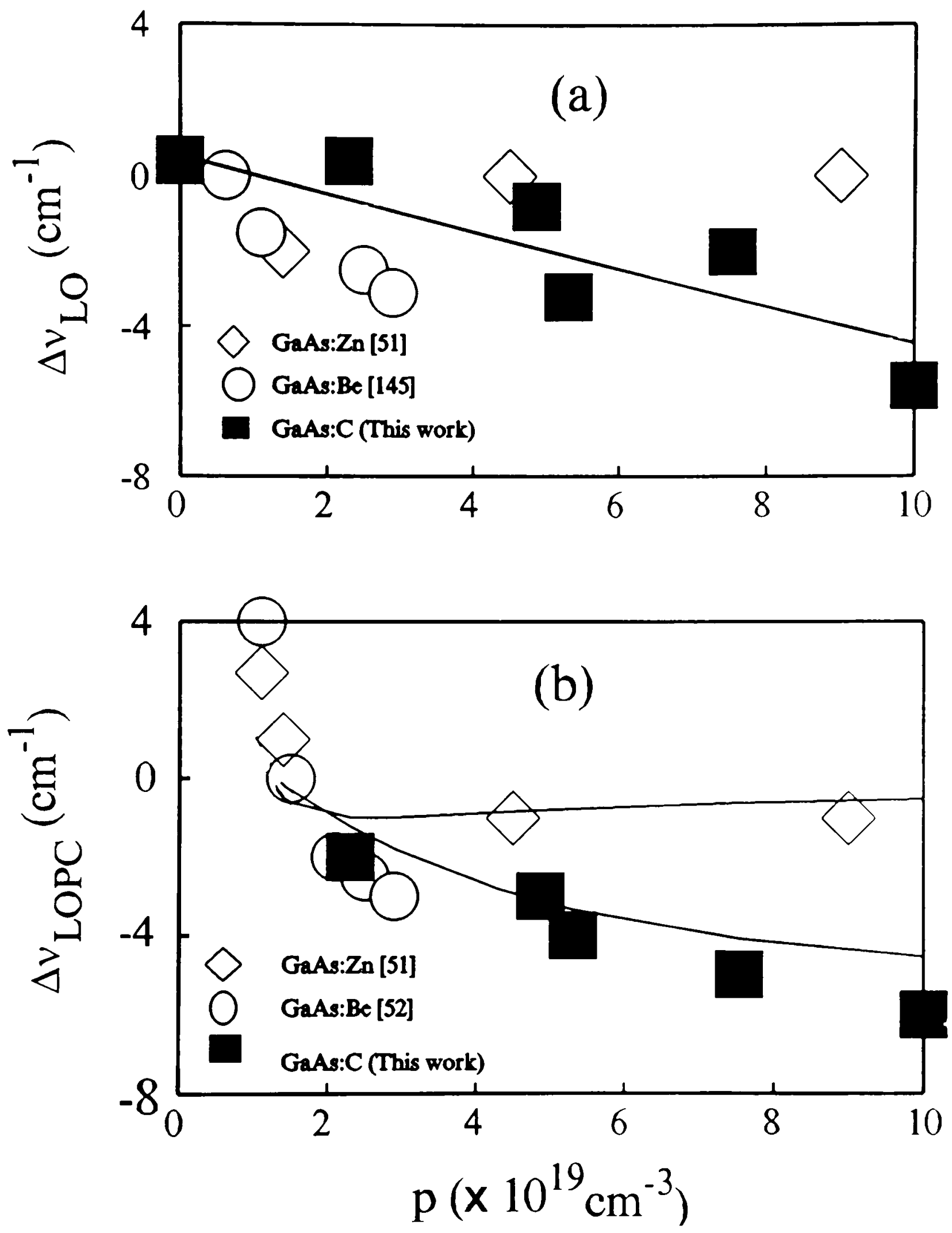

Figure 5.8: LO phonon (a) and LOPC band (b) positions isersus hole concentration (457.9 nm excitation). The solid squares represent our data while data for $\mathrm{Zn}$ and Be doped samples are included as diamonds and circles, respectively. (a) The solid line is a linear fit to the GaAs:C data. (b) The upper curve is from Eq. 2.24 with the parameter listed in Table 5.1. The lower curve includes the optic-phonon softening implied by the data in (a). 
where $p$ and $\mathrm{m}_{\mathrm{h}}{ }^{*}$ are the free hole density. and the average effective hole mass. In this form, $\varepsilon(\omega)$ accounts for one carrier type with plasma and phonon damping [51]. Solving for the roots of $\varepsilon(\omega)$ gives the frequencies of the LOPC modes. The real part of the complex root is the mode frequency and the imaginary part is the mode damping. not necessarily identical to $\gamma$ or $\Gamma$ [63]. Parameters used in solving the equation are summarized in Table 5.1. The average effective hole mass is estimated to be $m_{h}{ }^{*}=0.38 m_{0}$ by using [146]

$$
m_{l} *=\frac{m_{l l 1} * 32+m_{l l l} * 3 / 2}{m_{l l 1} * 12+m_{l l 1} * 1 / 2}
$$

with respective light and heavy hole masses in Table 5.1. The plasmon damping constant $\Gamma$ in Eq. 2.24 can be estimated from

$$
\Gamma=\frac{1}{\tau}=\frac{e}{m_{h}^{*} \mu}
$$

where $\tau$ is the carrier relaxation time averaged over all scattering mechanisms, and $\mu$ is the mobility of free carriers. The concentration and the mobility of the free carriers can be obtained by analyzing the frequency and bandwidth of the LOPC modes [62. 147]. In the case of $n$-type semiconductors no significant differences between the optically determined concentration $n$ and mobility $\mu$ and the results of electrical Hall and conductivity measurements were reported $[54,56,57.147]$. In $p$-type semiconductors the carrier concentration $p$ and the mobility $\mu$ differ from the electrically obtained values by

$$
\begin{aligned}
& p=\gamma_{H} p_{H} \\
& \mu=\frac{\mu_{H}}{\gamma_{H}}
\end{aligned}
$$


where $p_{\mathrm{H}}$ and $\mu_{\mathrm{H}}$ are the Hall carrier concentration and the Hall mobility, respectively, and $\gamma_{H}$ is the so-called Hall factor. Hall factor might be come from the fundamental differences between carrier mobility, which can be determined by an optical measurement, and Hall mobility [52]. That is, by electrical measurements only mobilityweighted carrier concentrations are obtained, whereas the optical characterization is sensitive to the total carrier concentration and to an average mobility. The discrepancy is attributed to the fact that the carrier mobility is calculated from average relaxation time which is a dynamical one, i.e., for $\omega \approx \omega_{p}$, whereas the Hall mobility is at $\omega=0$, that is static one $[61,62]$. When both acoustic phonon scattering and ionized impurity scattering mechanisms contribute to the Hall mobility, the resultant damping constant $\gamma_{H}$ is given by the following equation [56]:

$$
\gamma_{H}=\frac{3 \pi}{8} \frac{e}{m_{h}^{*} \mu_{a c}}+\frac{315 \pi}{512} \frac{e}{m_{h}^{*} \mu_{i}}
$$

where $\mu_{\mathrm{ac}}$ and $\mu_{\mathrm{i}}$ represent the Hall mobilities of acoustic phonon and ionized impurity scattering processes, respectively, when each mechanism is present alone. If the scattering by acoustic phonons is dominant, $\gamma_{\mathbf{H}}=3 \pi / 8 \approx 1$, and if the impurity scattering is dominant, $\gamma_{\boldsymbol{H}}=315 \pi / 512 \approx 2$. Irmer et al. [51] estimated the effective Hall factors 5.14, 2.02, and 1.11 for polar-optic mode scattering, deformation scattering, and ionized impurity scattering, respectively. In each case, only one dominant scattering mechanism is assumed. As shown from the above incoherent discussions on the Hall factor, the Hall factor for holes is difficult to measure or calculate. Effective Hall factors between 1 and 3.8 in p-type GaAs were theoretically expected and experimentally measured [61, 147 152]. In this study, a value of $\gamma_{\mathbf{H}}=1.9$ is used to explain data. The plasmon damping 
constant used in solving $\varepsilon(\omega)=0$ ranged between 560 and $690 \mathrm{~cm}^{-1}$, based on the effective hole mass carrier concentration dependence [153]. This effective mass increase with carrier concentration is discussed by Mlayah et al. in discussing beryllium doped GaAs system [62]. They attributed this behavior either to nonparabolicity of the valence bands structure since the Fermi level is below the top of the valence bands, or to changes in the band edge and density of states induced by carrier-carrier and carrier-dopant ion interactions $[18,62]$.

The curves in Fig. 5.8 (b) are calculated using Eq. 2.24. The upper curve is calculated using fixed $\omega_{\text {LO }}$ and $\omega_{\text {TO }}$ values (Table 5.1). This curve shows good agreement with the data of Irmer et al. for GaAs:Zn [51] but poor agreement with our data on GaAs:C and the GaAs:Be study of Fukasawa et al. [52]. We incorporate the systematic red shifting of the GaAs:C phonons in the lower curve in Fig. 5.8 (b). This is done for both the TO and LO phonons using the shift rate of the solid line in Fig. 5.8 (a) rather than fixed phonon energies in Eq. 2.24. Agreement with our data, and those from beryllium-doped $\mathrm{GaAs}$, is excellent.

The LOPC peak position, for varying $p$, is calculated by solving Eq. 2.24 for the roots of $\varepsilon(\omega)$. We obtain good agreement with Irmer et al. [51] for the LOPC positional dependence on $p$ with fixed $\omega_{\text {TO }}$ and $\omega_{\text {LO }}$. Their model determined the LOPC energy based on expressions for the calculated Raman intensity using both DP and Fröhlich scattering mechanisms. The good agreement between their calculation of the LOPC energy vs. $p$ and mine indicates that the dielectric function roots are adequate for modeling the LOPC mode position. 
Several factors influence the optic phonon (and consequently the LOPC) energies. The first factor is plasma screening of the macroscopic electric field produced by the L.O phonon [45]. At very high plasma frequencies the longitudinal field is screened and the LO phonon energy approaches that of the TO mode. This carrier screening effect is used to explain LOPC mode Raman shift in highly beryllium doped GaAs system by Mlayah et al. [62]. Even though carrier screening effect can explain LOPC mode red shift. it can not explain LO phonon mode red shift because this effect should not be a strong factor in the depletion region, where the plasma density is low. The second factor is a self-energ! effect [144] which has been used to describe the red shift seen in allowed scattering by TO phonons in heavily $\mathrm{Zn}$ doped GaAs. At low temperatures, this red shift is accompanied by a Fano lineshape to the TO band. The Fano effect stands for the change from symmetrical to asymmetrical phonon line shapes due to interference between a discrete state and a continuum. Examination of the allowed TO band, at reduced temperature, would be helpful to understand any similar effect in carbon doped GaAs. A third factor which can induce a phonon red shift is impurity scattering [144]. This effect can activate $\vec{k} \neq 0$ vibrational modes which are broadened, asymmetrical and shifted [62]. In our data, LO phonon and LOPC mode lineshapes are symmetric and do not vary for the carrier concentrations studied (Fig. 5.4). This implies that impurity scattering is weak in our samples. The fourth, and last factor which is naturally suggested by Fig. 5.8, is a dopant size effect. Since carbon and beryllium are both small atoms relative to gallium and arsenic, we interpret this as support of our suggestion that substitutionalatom size plays a role in the vibrational spectrum of GaAs when heavily doped with these elements. Estimation of the carbon size effect, assuming an analogy with GaAsN alloys 
[16] yields a total shift of $\approx-0.3 \mathrm{~cm}^{-1}$ at a carbon concentration of $1 \times 10^{20} \mathrm{~cm}^{-3}$. Since this only accounts for a small part of the needed $-5 \mathrm{~cm}^{-1}$ to describe our calculated curve in Fig. 5.8 (a), either the analogy with GaAsN is not correct or there are other factors influencing the observed red shift. Of these four factors. only the size effect is expected to be different when comparing carbon or beryllium doped GaAs with zinc as the dopant impurity. Unfortunately. the simple strain calculation does not adequately describe the observed red shift in the LO and LOPC bands. We cannot rule out the possibility that the size effect amplifies one or more of the other factors. such as impurity scattering. through local strain. However. we have no further evidence that this is the case. The differences between the data sets for small and same-size impurities in GaAs. as summarized in Fig. 5.6, require further work to become better established and understood.

We now turn our attention to the intensity of the LOPC mode. Fig. 5.3 presents $\mathrm{I}_{\mathrm{LOPC}} / \mathrm{I}_{\mathrm{LO}}$ data versus $p$ on a $\log -\log$ scale. Also included in Fig. 5.3 are data from GaAs doped with beryllium [52.62,65] and germanium [67] each in the same hole concentration range. Agreement between the trend in Fig. 5.3 at high carrier concentrations and in the lower range further substantiates our identification of the band near $270 \mathrm{~cm}^{-1}$ as the LOPC mode. At low $p$ the TO phonon and LOPC band are fully resolvable. Thus, the intensity rise with carrier concentration below $\sim 10^{19} \mathrm{~cm}^{-3}$ is not enhanced TO phonon scattering. Although the TO and LOPC are not resolved at high $p$, there is no reason to expect any enhancement of the TO band to follow the same intensity trend as the LOPC line. I note here that the LOPC in zinc doped GaAs showed the same trend as the data in Fig. 5.3, slightly displaced to higher LOPC intensities [51, 66]. Variations between data sets may stem from the variety of excitation photon energies 
used in the literature and polarization configurations. These data establish the intensity ratio $\mathrm{I}_{\mathrm{LOPC}} / \mathrm{I}_{\mathrm{LO}}$ as a non-destructive, Raman (optical) method for assessing high hole concentrations in GaAs. This is far superior to using the LVM intensity due to the comparatively strong intensities of the first-order Raman spectrum.

The basic agreement between the LVM intensity and $\mathrm{I}_{\mathrm{LOPC}} / \mathrm{I}_{\mathrm{LO}}$ dependence on $p$ in Fig. 5.3 indicates that the latter ratio is directly proportional to carrier concentration. Our LVM intensity results suggest that the LO phonon intensity varies little over the range of $p$ we studied. This weak dependence of $\mathrm{I}_{\mathrm{LO}}$ on $p$ (for high $p$ ) has been reported previously for GaAs:Zn [66] and GaAs:Be [137] and will be discussed in section 5.3.

\subsection{Discussion of the LO mode intensity}

The above arguments rely on the use of the LO phonon as an internal intensity standard. That is, $\mathrm{I}_{\mathrm{LO}}$ is independent of $\mathrm{p}$ for $p>1 \times 10^{19} \mathrm{~cm}^{-3}$. In heavily doped $\mathrm{p}$-type $\mathrm{GaAs}$, the Fermi level in the epilayer is very close to or below the valence band maximum. The Fermi level at the surface is pinned at the mid gap due to surface states, leading to band bending [12] as shown in Figure 5.9. The surface band bending results from the fact that in equilibrium the Fermi level must remain constant from the epilayer to surface $[1,12]$. The band edge must then vary as depicted in the Fig. 5.9, resulting in a surface hole depletion region in this case. It is generally accepted that the bare LO phonon intensity stems from the hole-depletion region. For our samples, we estimate that hole-depletion region thickness, $\mathrm{W}$, ranges from 3.1 (for $p=1 \times 10^{20} \mathrm{~cm}^{-3}$ sample) to 5.8 $\mathrm{nm}$ (for $p=1 \times 10^{20} \mathrm{~cm}^{-3}$ sample) based on Eq. 5.1. As for the low frequency dielectric constant, $\varepsilon_{0}=12.85$ is used [142]. The surface band bending $V_{B}$ was calculated based on 


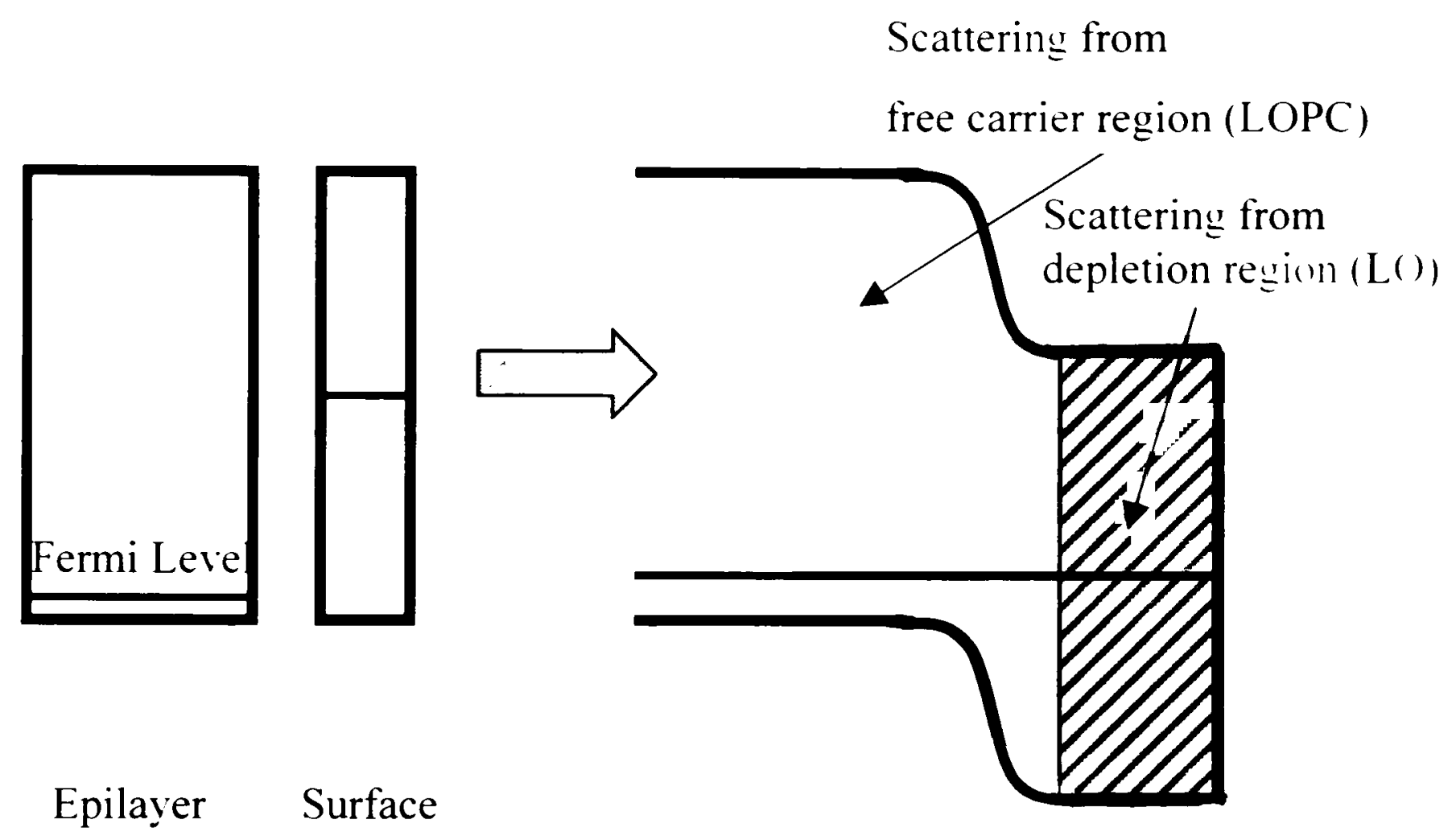

Equilibrium Condition

Figure 5.9: The Fermi level at the epilayer is very close to or under the top of valence band. and is pinned at the midgap at the surface. The Fermi level should be a straight line under equilibrium condition. resulting in the depletion region near the surface. 
the values of Fermi level at the surface which is $0.5 \mathrm{eV}$ from the top of valence band [154] and bulk Fermi level position for different carrier concentrations [76, 155]. Thus, the intensity should have a scattering-volume (length) term

$$
I_{1.0} \propto \int_{0}^{1} e^{-2 \alpha z} d z \approx W
$$

where $\alpha=\alpha\left(\hbar \omega_{1}\right) \approx \alpha\left(\hbar \omega_{s}\right)$ is the optical absorption coefficient at the incident- and scattered-light photon energies [118]. The final approximation in Eq. 5.6 is for $\mathrm{W}<<$ $1 /(2 \alpha)$. If this relation holds, then $I_{L O}$ should diminish by a factor of $\approx 2$ over our range of $p$. Evidently. variation in the depletion-region thickness is not sufficient to influence the LO band Raman intensity. Indeed, measurements of the absolute LO phonon intensity in GaAs:Zn show only a weak dependence on $p$ in the $1 \times 10^{19}$ to $1 \times 10^{20} \mathrm{~cm}^{-3}$ range [66]. The $\mathrm{I}_{\mathrm{LO}}$ saturation is also observed from Be doped GaAs [137] consistent with our conclusion that the depletion width saturates at high $p$. This limitation on the depletion width may be partially attributed to surface effects which become important with such narrow hole-depletion regions. Surface states (of the GaAs or the native oxide), for example, may play an influential role in determining the nature of the hole-depletion region for extremely small values of $\mathrm{W}$.

\subsection{Raman spectroscopy from annealed GaAs: $\mathrm{C}$}

Raman spectra are taken from annealed samples. Figure 5.10 compares Raman spectra taken from unannealed and annealed $p=4.6 \times 10^{19} \mathrm{~cm}^{-3}$ samples. Samples are annealed during 1 hour at different temperatures ranging from 350 to $850{ }^{\circ} \mathrm{C}$. Raman spectrum from unannealed $p=4.6 \times 10^{19} \mathrm{~cm}^{-3}$ sample shows LO and LOPC mode peaks 


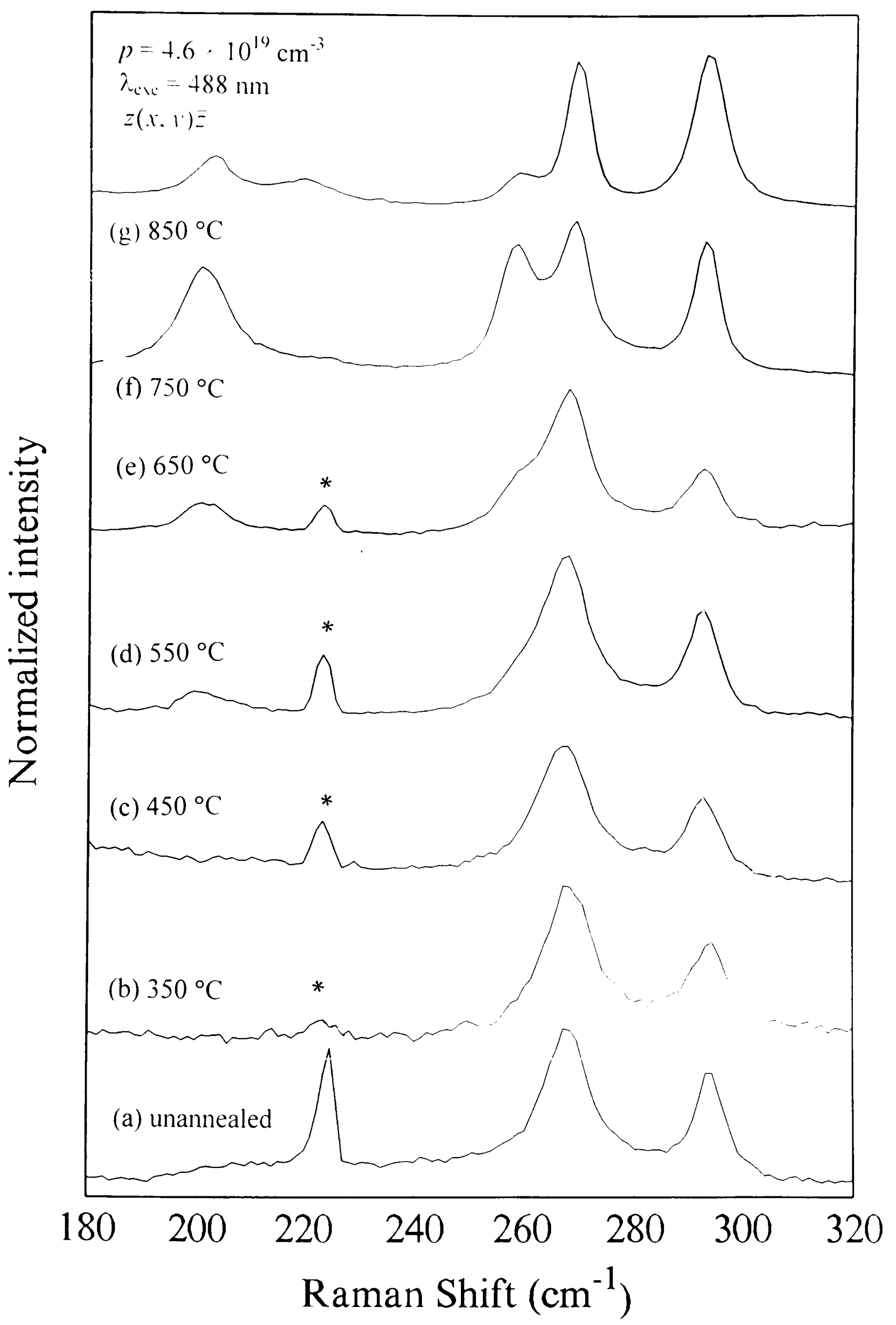

Figure 5 10: Room-temperature Raman spectra taken from (a) unannealed and (b) (g) annealed $p=4.6 \times 10^{19} \mathrm{~cm}^{-3}$ samples. Samples are annealed between $\left.3500^{\circ} \mathrm{C}-8.50\right)^{\circ} \mathrm{C}$ during I hour. Peaks $w$ ith * sign are Ar plasma lines. 
and another near $223 \mathrm{~cm}^{-1}$. The peak near $223 \mathrm{~cm}^{-1}$ is one of Ar plasma lines used for calibration.

The series of Raman spectra in Fig. 5.10 shows very interesting features. From the low wavenumber, the intensity and/or FWHM of 200, 223, 258, and $270 \mathrm{~cm}^{-1}$ peaks show strong dependence on annealing temperature. The intensity of 200 and $258 \mathrm{~cm}^{-1}$ peaks increases as annealing temperature, $T_{a}$, increases up to $750^{\circ} \mathrm{C}$. The intensity decreases if $T_{a}$ is higher than that. The peak near $223 \mathrm{~cm}^{-1}$ is broadened at high $T_{a}$ Raman spectra as shown in Fig. 5.10 (f) and (g). The FWHM of peak near $270 \mathrm{~cm}^{-1}$ decreases at high temperature annealing as shown in Fig. 5.10 (a) and (g), and the position of the peak is blueshifted at high $\mathrm{T}_{\mathrm{a}}$. The peaks at $200 \mathrm{~cm}^{-1}$ and $258 \mathrm{~cm}^{-1}$ are suggested as arising from microcrystalline arsenic which is clustered upon annealing between 300 and $600{ }^{\circ} \mathrm{C}$ [156]. When samples are annealed, Ga is preferentially oxidized, leaving As behind [157]. This might be due to not enough purging the annealing chamber before annealing. Crystalline As has rhombohedral structure and $D_{3 d}$ symmetry [157]. The peak identification is based on comparison between various III-V semiconductor Raman spectra, polarization selection rule and peak position. The frequencies of the peaks are almost the same as those for the doubly degenerate $E_{\mathrm{g}}$ modes $\left(195 \mathrm{~cm}^{-1}\right)$ and the $A_{i g}$ mode $\left(257 \mathrm{~cm}^{-1}\right)$ of crystalline arsenide. The weakening of the peaks at $200 \mathrm{~cm}^{-1} 258 \mathrm{~cm}^{-1}$ was observed after the $600{ }^{\circ} \mathrm{C}$ annealing [156]. The peak around $223 \mathrm{~cm}^{-1}$ in Fig. 5.10 (c) $\sim$ (e) is considered as one of Ar plasma lines as identified in Fig. 5.10 (a). However, the peak in Fig. $5.10(\mathrm{f})$ and $(\mathrm{g})$ is not due to Ar plasma lines. The peak is attributed to the mode of As precipitate [156]. It is very interesting that the weakening of 200 and 258 $\mathrm{cm}^{-1}$ peaks happens synchronously with the strengthening of $223 \mathrm{~cm}^{-1}$ peak. This 
suggests that the As cluster makes precipitate as $T_{a}$ increases. Finally, the peak near 270 $\mathrm{cm}^{-1}$ in Fig. $5.10(\mathrm{~g})$ is considered as mainly forbidden TO phonon, even though the peak near $270 \mathrm{~cm}^{-1}$ in Fig. 5.10 (b) is the LOPC mode. This identification is based on the FWHM and position of the peak, and comparison with Raman spectrum taken from annealed pure GaAs as shown in Fig. 5.11.

$\mathrm{I}_{\mathrm{LOPC}} / \mathrm{I}_{\mathrm{LO}}$ is calculated from annealed samples. As mentioned before, the forbidden TO phonon mode intensity increases as $T_{a}$ increases. Therefore, it should be subtracted from the $\approx 270 \mathrm{~cm}^{-1}$ peak to get $\mathrm{I}_{\mathrm{LOPC}} / \mathrm{I}_{\mathrm{LO}}$. It is done by fitting Raman spectra as shown in Fig. 5.12. $\mathrm{I}_{\mathrm{CAS}} / \mathrm{I}_{\mathrm{LO}}$ and $\mathrm{I}_{\mathrm{LOPC}} / \mathrm{I}_{\mathrm{LO}}$ are both decreased from annealing as shown in Figures 5.13 and 5.14. It is not clear whether the As clustering and/or precipitation shown in Fig. 5.10 is related to the reduction of $I_{C A s} / I_{L O}$ and $I_{L O P C} / I_{L O}$ because the reduction is also observed even when As cluster and/or precipitate peak is very weak as shown in figure 5.13. $\mathrm{I}_{\mathrm{LOPC}} / \mathrm{I}_{\mathrm{LO}}$ decrease was also observed when samples are exposed to strong incident laser light. To see if this change is reversible or not, samples are intentionally exposed to strong incoming light, and then, the incoming light was blocked for 30 minutes. The initial ratio was not recovered. After this observation, the laser power was limited to low $(\approx 20 \mathrm{~mW}$ on a spot $\sim 10 \mu \mathrm{m}$ diameter $)$. Even though $\mathrm{I}_{\mathrm{LOPC}} / \mathrm{I}_{\mathrm{LO}}$ decreases for three different samples, the decrease is more drastic for higher carrier density. Because the ratio change for lower carrier concentration is small, it seems like that $\mathrm{I}_{\mathrm{LOPC}} / \mathrm{I}_{\mathrm{LO}}$ is approaching a certain value. As suggested in section $5.2, \mathrm{I}_{\mathrm{LOPC}} / \mathrm{I}_{\mathrm{LO}}$ is proportional to carrier concentration (Fig. 5.3). Therefore, the observed behavior of $\mathrm{I}_{\mathrm{LOPC}} / \mathrm{I}_{\mathrm{LO}}$ mentioned above implies that carrier density reduction is enhanced for high hole density samples, and the carrier concentration of annealed sample approaches to a limiting $p$ of $6 \times 10^{18}$ 


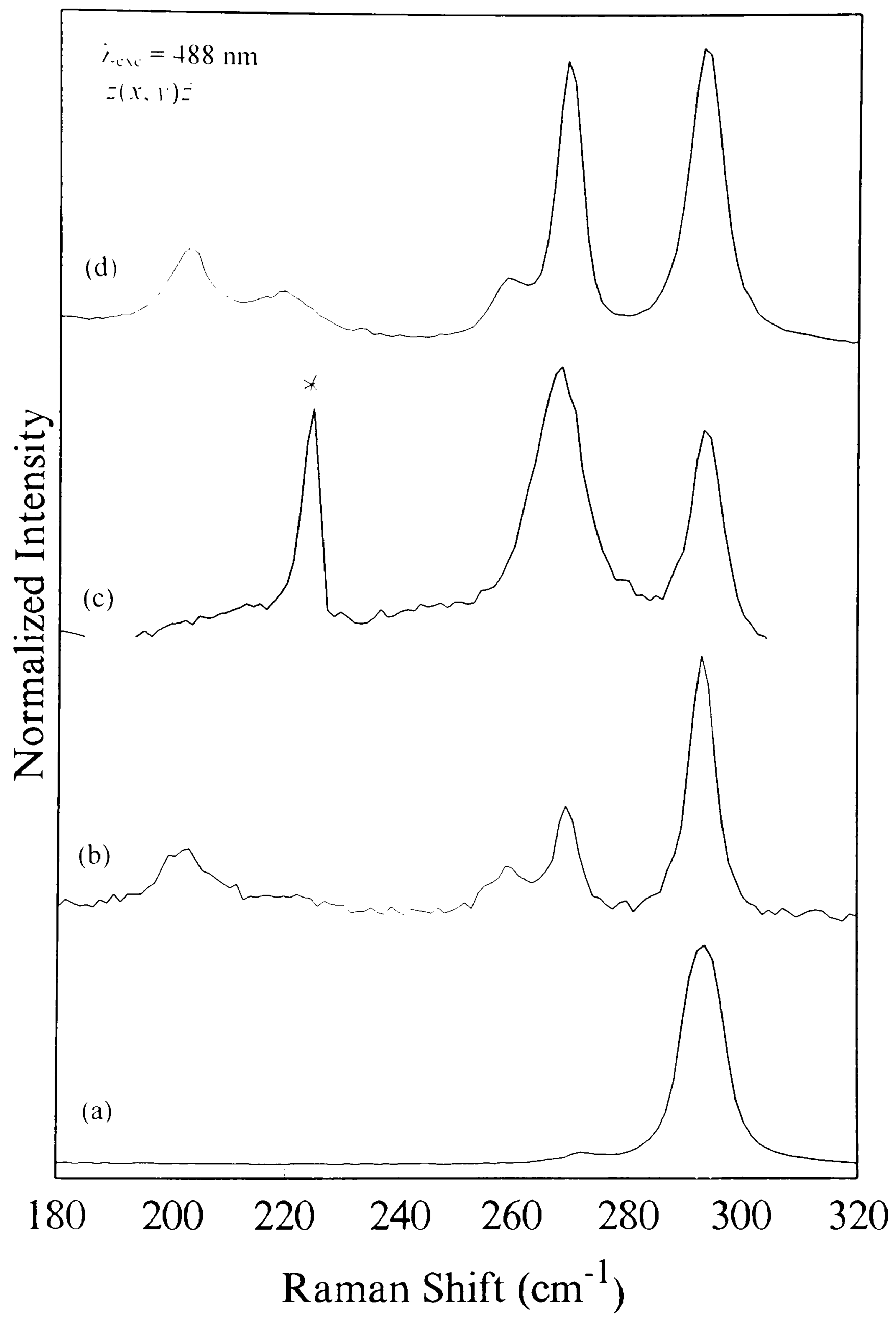

Figure 5 11: Raman spectra taken from (a) unannealed and (b) annealed bulk (ials and (c) unannealed and (d) annealed $p=4.6 \times 10^{14} \mathrm{~cm}^{-3}$ samples. Annealing condition is 8511 "C and 1 hour. 


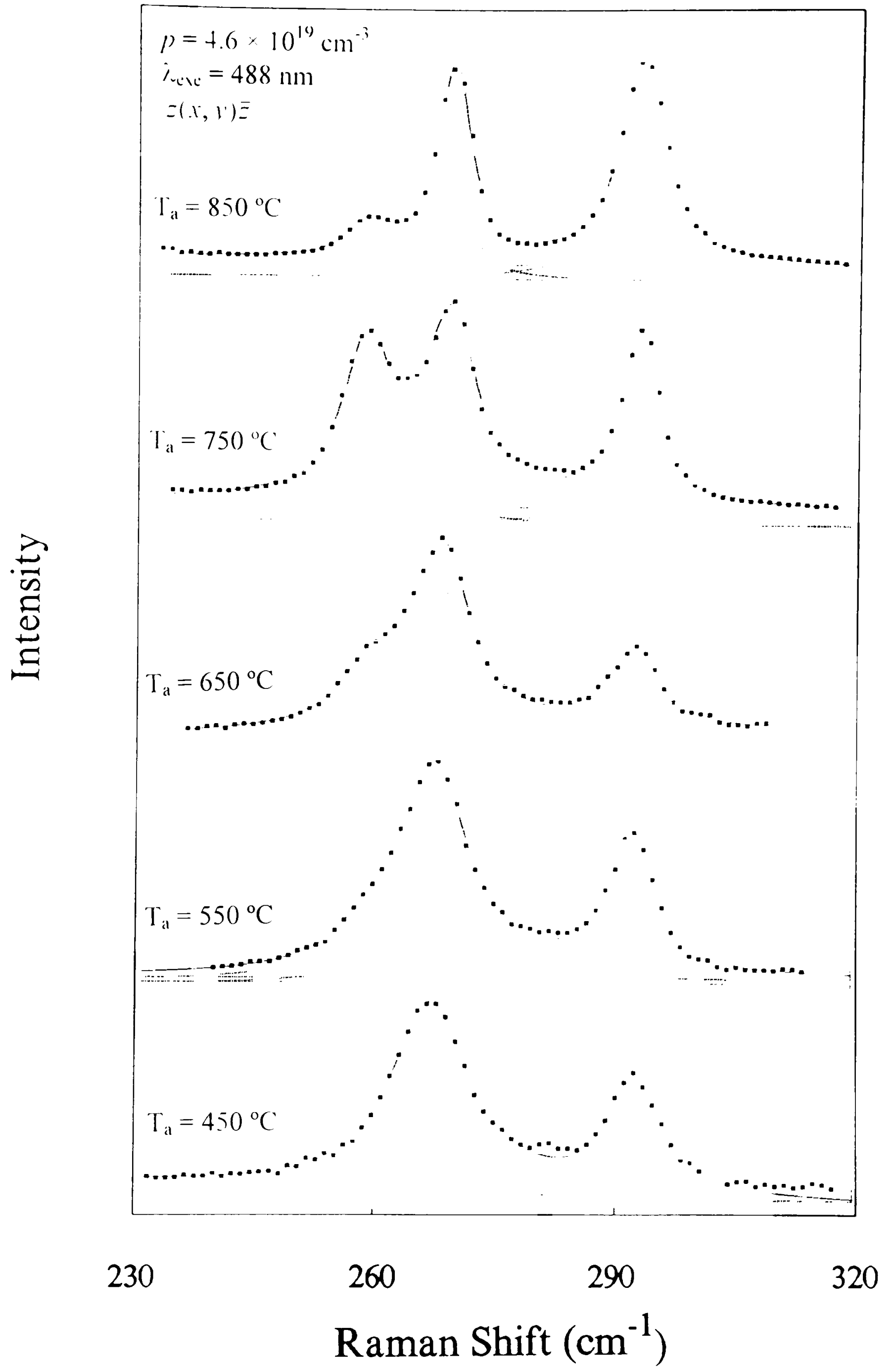

Figure 5.12: Raman spectra taken from annealed $p=4.6 \times 10^{19} \mathrm{~cm}^{-3}$ samples. Sample are annealed 1 hour at different temperatures. Spectra are fitted with $2 \sim+$ peaks 1 calculate $\mathrm{I}_{1}$ ope $\mathrm{l}_{1}$ o ratio. 


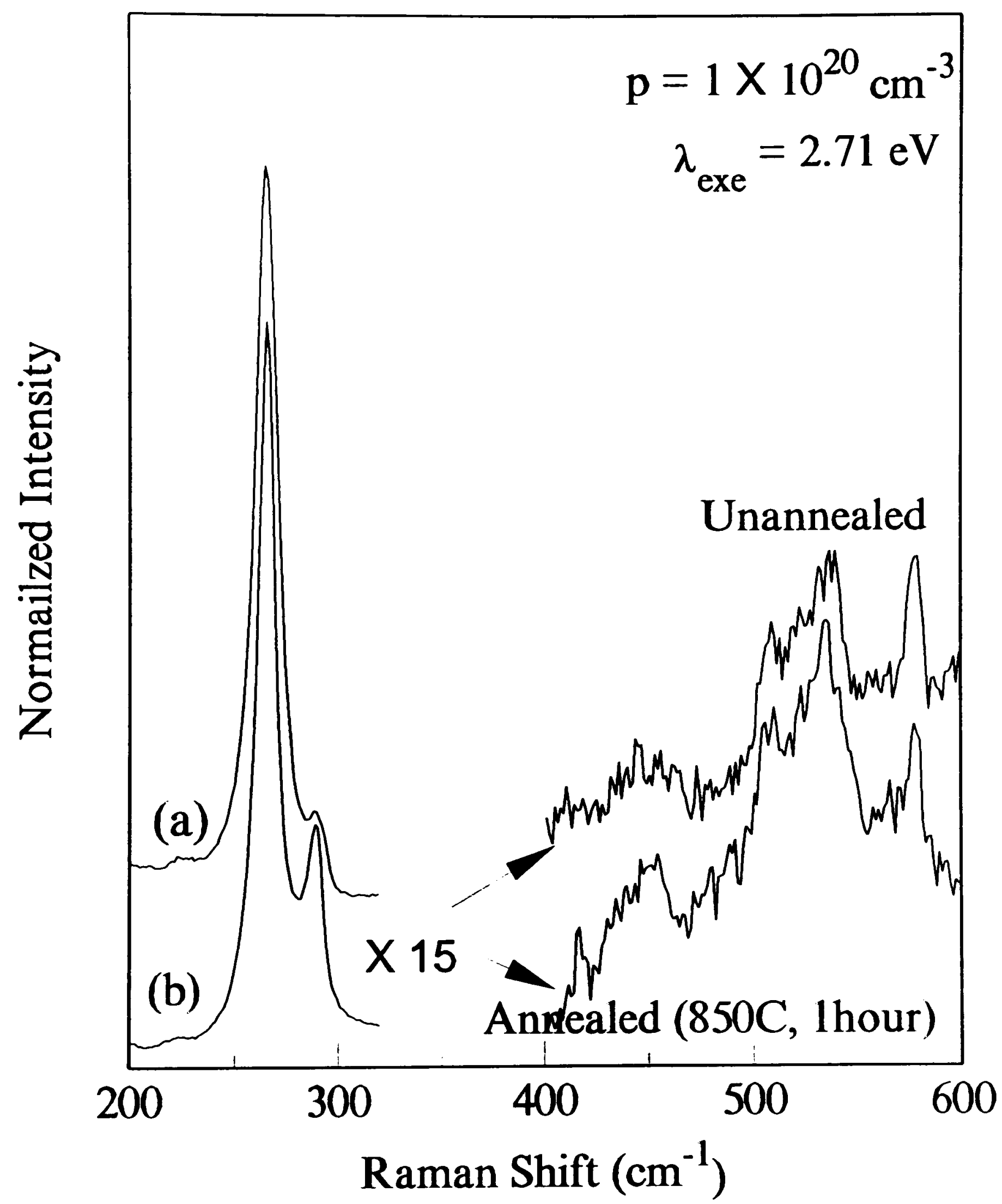

Figure 5.13: Raman spectra taken (a) unannealed and (b) annealed $p=1 \times 10^{20} \mathrm{~cm}^{-3}$ samples. $I_{L O P C} / I_{L O}$ and $I_{L V M} / I_{L O}$ decrease upon annealing. 


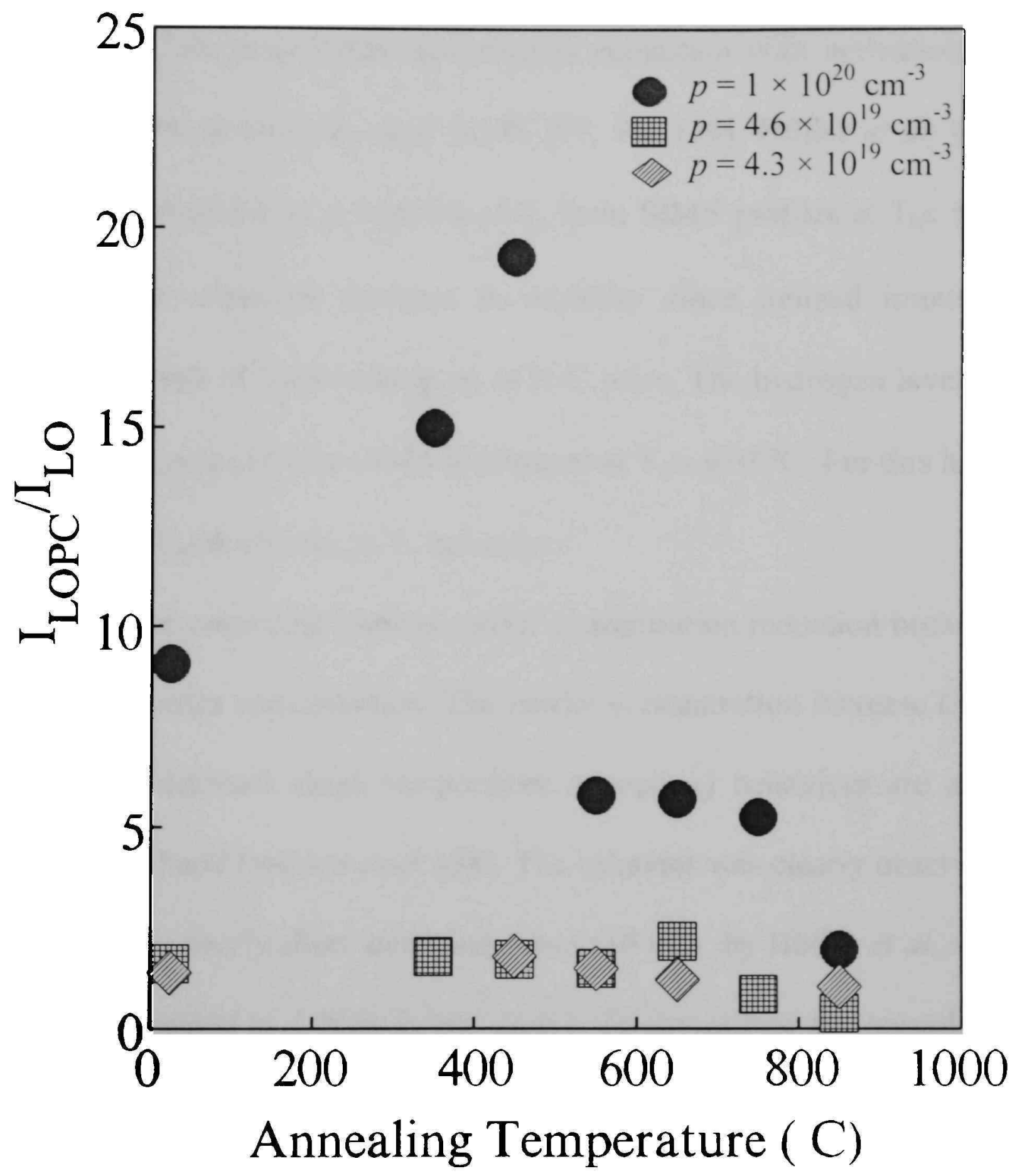

Figure 5 14: $\mathrm{I}_{\mathrm{LOPC}} / \mathrm{I}_{\mathrm{LO}}$ calculated from unannealed and annealed samples. 
$\mathrm{cm}^{-3}$. This behavior was observed by many other studies $[97,100,101,103]$. even though different limiting carrier concentrations are suggested from these studies, ranging from low $10^{19} \mathrm{~cm}^{-3}[101]$ to $6 \times 10^{19} \mathrm{~cm}^{-3}[100] . \mathrm{I}_{\mathrm{LOP}} / \mathrm{I}_{\mathrm{lO}}$ normalized with respect to that of unannealed sample is plotted as a function of annealing temperature in Figure 5.15 . $\mathrm{I}_{\mathrm{LOPC}} / \mathrm{I}_{\mathrm{LO}}$ is increased when the samples are annealed at low temperatures. This carrier concentration increase upon low temperature annealing is consistent with activation of carbon tied up in $\mathrm{H}_{-} \mathrm{C}_{\mathrm{As}}$ pairs, as studied using SIMS $[97.98,158]$. Höfler 't al. and Fushimi et al. observed $[\mathrm{H}]$ decrease as a function of $\mathrm{T}_{\mathrm{a}}$ from SIMS profiles at $\mathrm{T}_{\mathrm{a}}<550$ ${ }^{\circ} \mathrm{C}$. They also observed the expected decrease in mobility since ionized impurity scattering is increased as a result of the breaking up of $\mathrm{H}-\mathrm{C}$ pairs. The hydrogen level in the epilayer reached the background of the SIMS instrument at $\mathrm{T}_{\mathrm{a}}>650^{\circ} \mathrm{C}$. For this high annealing temperature, $\mathrm{I}_{\mathrm{LOPC}} / \mathrm{I}_{\mathrm{LO}}$ decreases as $\mathrm{T}_{\mathrm{a}}$ increases.

$\mathrm{I}_{\mathrm{LOPC}} / \mathrm{I}_{\mathrm{LO}}$ decrease upon annealing implies carrier concentration reduction because $\mathrm{I}_{\mathrm{LOPC}} / \mathrm{I}_{\mathrm{LO}}$ is proportional to carrier concentration. The carrier concentration increase (low temperature annealing) and decrease (high temperature annealing) behaviors are also published by Höfler et al. [97] and Fushimi et al. [98]. The behavior was clearly observed even though they used comparatively short annealing times $(10 \mathrm{~min}$. by Höfler $\mathrm{et} \mathrm{al}$. and 3 min. by Fushimi et al. compared to 1 hour in my study). To investigate the annealing time effect on carrier concentration reduction, different anneal times (20 minutes and 1 hour) are used for each $650{ }^{\circ} \mathrm{C}$ and $850{ }^{\circ} \mathrm{C}$ annealing. Even though $\mathrm{I}_{\mathrm{L}, O P C} / \mathrm{I}_{\mathrm{LO}}$ decreases are observed in all cases, it did not show any trend. These observation suggests that a significant decrease in hole concentration occurs at the early stage of anneal, and then a decrease slows down as the anneal proceeds [101]. 


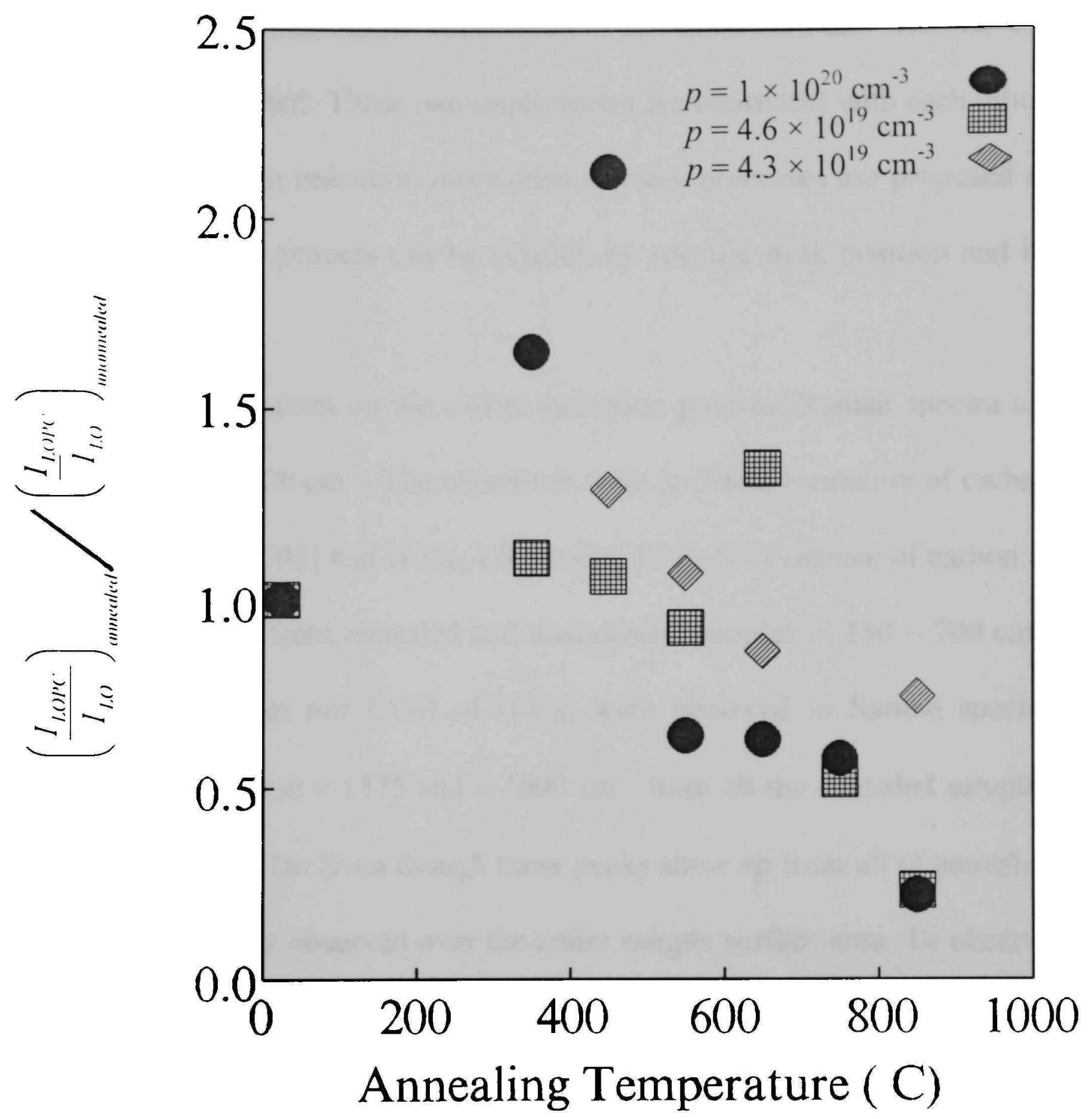

Figure 5.15: Normalized $\mathrm{I}_{\mathrm{LOPC}} / \mathrm{I}_{\mathrm{LO}}$ with respect to that of unannealed samples. 
In Figure 5.13, $\mathrm{C}_{\mathrm{As}}$ modes are shown with LOPC modes. As LOPC mode intensity decreased, $I_{C A s}$ is also seen to decrease in the annealed samples. These measurements imply that the free-carrier concentration has decreased and that the $C_{A s}$ concentration has also diminished. These two implications are consistent with each other. As for the carrier concentration reduction mechanisms, many processes are proposed as described in section 2.7. Each process can be verified by specific peak position and its polarization selection rule.

To obtain more information on the carrier reduction process, Raman spectra are taken in the range of $150 \sim 2000 \mathrm{~cm}^{-1}$. The objectives were to detect formation of carbon precipitations [102] and C-C [105] and $\mathrm{H}-\mathrm{C}_{\mathrm{As}}$ LVMs [39. 109]. No evidence of carbon at gallium site LVM is observed from annealed and unannealed samples in $150 \sim 700 \mathrm{~cm}^{-1}$ range. Neither di-carbon center nor LVM of $\mathrm{H}-\mathrm{C}_{\mathrm{As}}$ were observed in Raman spectra taken in this study. Two peaks at $\approx 1375$ and $\approx 1600 \mathrm{~cm}^{-1}$ from all the annealed samples showed up as seen in Figure 5.16. Even though those peaks show up from all of annealed samples, they are not uniformly observed over the entire sample surface area. To observe the peaks, sample surfaces are inspected with microscope and the incident beam is focused on black pits seen on the annealed sample surface (Fig. 4.4 (d)). This is different from what is observed for $I_{L O P C} / I_{L O}$. Decrease in this latter quantity is observed uniformly from the annealed sample surface. The intensity and FWHM of the $\approx 1375$ and $\approx 1600$ $\mathrm{cm}^{-1}$ peaks vary significantly from spot to spot, especially for the peak $\approx 1375 \mathrm{~cm}^{-1}$. Because of significant spot to spot fluctuation of the intensity and feature width, it was 


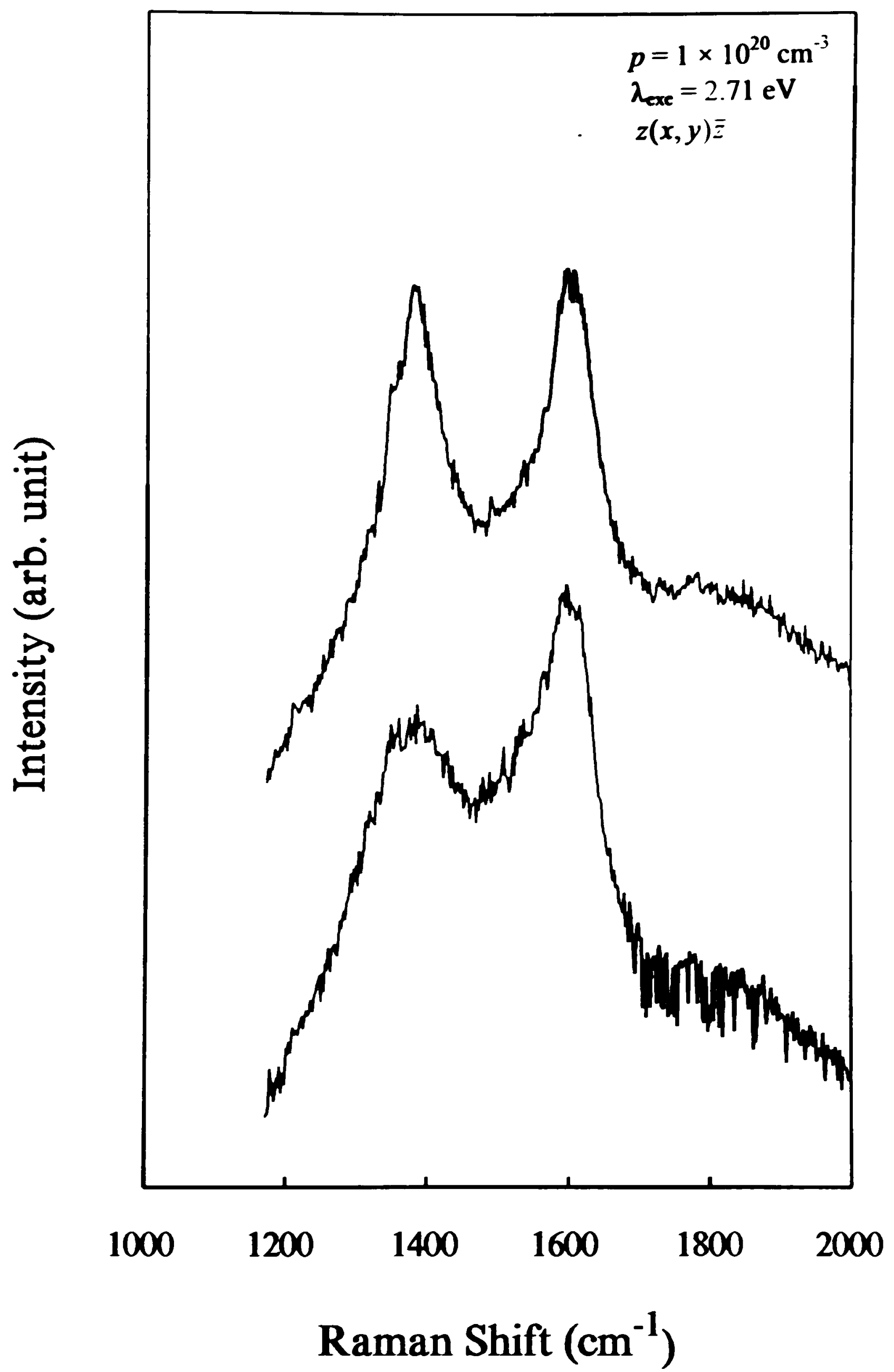

Figure 5.16: Raman spectra taken from $p=1 \times 10^{20} \mathrm{~cm}^{-3}$ sample at different spot. Sample is annealed at $850^{\circ} \mathrm{C}, 1$ hour. 
hard to extract any trend on intensity and linewidth variation as a function of carrier concentration or annealing conditions. The two strong peaks around 1585 and $1355 \mathrm{~cm}^{-1}$ are unambiguously assigned to $\mathrm{C}-\mathrm{C}$ precipitating in the $\mathrm{sp}^{2}$ phase [102]. Two peaks are designated as the D-band (centered between 1340 and $1390 \mathrm{~cm}^{-1}$ ) and G-band (centered between 1540 and $1590 \mathrm{~cm}^{-1}$ ), and correspond to the nano-crystalline graphite phase [119]. Crystalline graphite has two $\mathrm{E}_{2 \underline{\mathrm{g}}}$ Raman active vibrational modes. One of the $\mathrm{E}_{2 \underline{\mathrm{g}}}$ modes is due to stretching of the in-plane bonds, resulting in a vibration at $1581 \mathrm{~cm}^{-1}$. Finite-size disorder in graphite will be evident in Raman spectrum in several ways. The first is a broadening of the $1581 \mathrm{~cm}^{-1}$ mode and an upshift towards $1600 \mathrm{~cm}^{-1}$. The second is the emergence of a band at $\sim 1350 \mathrm{~cm}^{-1}$, believed to correspond to a zone-edge vibration mode $\left(\mathrm{A}_{\mathrm{lg}}\right)$. These peaks are extensively studied. and summarized by Dennison [15.9]. From the above observations, it is believed that carbon precipitates into a nanocrystalline graphite phase upon annealing. The crystal size of the carbon precipitates can be estimated from the Raman spectra. The integrated intensity ratio of the $D$ and $G$ bands, $I_{D} / I_{G}$, is a function of $\mathrm{sp}^{2}$ domain size in disordered graphite. An inverse relationship between domain size, $L_{\mathrm{a}}$, and $\mathrm{I}_{\mathrm{D}} / \mathrm{I}_{\mathrm{G}}$ ratio in microcrystalline graphite has been found by Tuinstra and Koenig [160] using combined Raman and X-ray studies:

$$
L_{a}(n m)=4.4 \times\left(\frac{I_{1)}}{I_{(i}}\right)^{-1} .
$$

The size of the nanocrystalline graphite can be estimated by using D and G integrated intensity band ratio, $I_{G} / I_{D}$, based on Eq. 5.7. Graphite domain size is calculated between 3.8 and $7.9 \mathrm{~nm}$ from the ratio ranging from 0.86 to 1.8 for annealed samples. 
The reduction of hole density identified from $I_{L_{. O P C}} / \mathrm{I}_{\mathrm{I}_{(O)}}$ decrease in annealed samples implies a decrease of the carbon-at $A$ s site population. It is suggested that the migrated carbon is responsible for the nanocrystalline graphite, as shown in Fig. 5.16. Due to this migration, carrier concentration reduction is observed through out the sample area. This migration is accelerated at high temperature annealing, resulting in bigger carrier concentration reduction. The process is considered as non-reversible.

\subsection{Summary}

The $\mathrm{C}_{\mathrm{As}}$ LVM (Figures 5.1 and 5.2) is found to have an intensity directly proportional to $p$, consistent with what is expected from Eq. 2.4. Both the LOPC mode and LO phonon are investigated in the optic-phonon regime. The LO phonon mode is attributed to coming from the depletion region. The LOPC mode showed symmetric shape in the carrier concentration range studied here. This is different from what was observed for $p \leq 2 \times 10^{19} \mathrm{~cm}^{-3}$ samples from other studies [51,59,62, 144]. The lineshape of the LOPC band has been calculated by using the DP and electron-phonon interaction mechanisms $[51,59,62,64,144]$. The calculated LOPC mode shape was asymmetric at low carrier concentration and more symmetric at high carrier concentration. Only one LOPC mode shows up in calculated LOPC lineshape under high plasmon damping condition. The calculated LOPC band shape shows experimentally observed behavior.

In our measurements for very high carrier concentrations the LOPC lineshape shows no systematic variation with carrier concentration. This is consistent with what is observed and expected based on other studies $[51,62,66,145]$. Therefore I focus on the 
LOPC mode energy which shows a systematic shift in my experiments because no systematic information can be drawn from the lineshape analysis. Both of LO and LOPC the peaks in Figs. 5.4 and 5.8 redshift between carrier concentrations of $2.3 \times 10^{19}$ and 1 $\times 10^{20} \mathrm{~cm}^{-3}$. The LOPC peak position, for rarying $p$. is calculated by solving Eq. $2.2+$ for the roots of $\varepsilon(1))$. We obtain good agreement with Irmer et al. [51] for the LOPC positional dependence on $p$ with fixed $\omega_{\text {TO }}$ and (1)LO. Therefore. the LOPC band red shift is adequately described by a dielectric function which includes phonon and free carrier contributions. However, it failed to explain the LOPC mode redshift observed at very high carrier concentration in GaAs:Be [52.145] and GaAs:C. Moreover, the red shift of the LO band is not fully described by the influence of high hole concentrations on the dielectric function [67]. We attribute the difference to internal strain due to the relative smallness of dopants in the GaAs crystal. We must include. thereforc. the softening of the optic phonon branches implied by the position of the LO phonon. By examining the relative intensities of the LOPC band and LO phonon, we find that $I_{I O P C} / I_{L O}$ is in proportion to $p$. This implies that the relatively straightforward measurement of this intensity ratio can be used to estimate the carrier concentration.

The elevated temperature is inevitable in device processing, and capable of altering its properties unexpectedly. To understand these phenomena, several anneals are conducted. $\mathrm{I}_{\mathrm{LOPC}} / \mathrm{I}_{\mathrm{LO}}$ decreases upon annealing. implying the carrier concentration reduction. To verify the suggested carrier concentration reduction mechanisms in section 2.7, Raman spectra are taken in the range of $150 \sim 2000 \mathrm{~cm}^{-1}$. None of carbon at gallium site LVM. di-carbon center, and LVM of $\mathrm{H}-\mathrm{C}_{\mathrm{A}}$, was observed. Two peaks at $=1375$ and $=1600 \mathrm{~cm}^{-1}$ from all the annealed samples showed up as seen in Fig. 5.16. The two peaks 
are assigned to C-C precipitates. From the observation, it is believed that carbon precipitates into a nanocrystalline graphite upon annealing. The crystal size of the carbon precipitates was estimated from the peak intensity ratio, $\mathrm{I}_{1375 \mathrm{~cm}-1} / \mathrm{I}_{1600 \mathrm{~cm}-1}$, and Eq. 5.7 . High temperature $\left(>600^{\circ} \mathrm{C}\right)$ anneals thus produce several deleterious side effects: Carrier-concentration reduction, arsenic precipitation, and carbon precipitation. While nitride or oxide caps can limit As expulsion from the GaAs. it is not known if carbon will also be kept from leaving the crystal or, more importantly, from leaving their arsenic sites. 


\section{CHAPTER VI}

\section{SUMMARY}

To characterize samples, photoluminescence and Raman spectroscopy are used. PL study was done to get a better characterization of samples and to see if some complementary chemical level information could be obtained to compare with Raman spectra. Photoluminescence spectra in $1.3 \sim 1.6 \mathrm{eV}$ range at $77 \mathrm{~K}$ show four peaks. Peak around $1.508 \mathrm{eV}$ is considered as conduction band-to-valence band emission from the - substrate. As carrier concentration increases, the intensity of the peak decreases due to the carrier concentration dependent band gap energy and the energy gap dependent absorption coefficient. Peak B in Fig. 4.1 is attributed to conduction band-to-acceptor level emission from the peak position and carrier concentration dependent intensity. As carrier concentration increases, the width of peak B increases. From the low-energy tail edge the band gap energy is calculated. The band gap shrinkage in this study shows good agreement with the results of Borghs et al. [77]. A shoulder at the high-energy side of peak B is observed only from most highly doped sample. Therefore, no clear identification on the peak can be made in this study without the information on carrier concentration dependence of peak position. The high-energy tail in PL spectra is attributed to hot-electron PL.

Many annealing treatments are inevitable during device processing. To investigate how these treatments affect the physical properties of heavily carbon doped GaAs, annealing study is conducted. PL spectra taken from annealed samples show drastic PL 
intensity decrease. This is attributed to hole concentration reduction and introduction of nonradiative recombination centers like carbon precipitates.

Raman spectroscopy is taken to investigate lattice vibrations. which is very sensitive to local structure. long-range crystalline order. and the effects of doping and their interactions with other excitations such as plasmon. Raman spectra from heavily carbon doped GaAs show carbon at arsenic local vibrational mode. LO phonon and LO phonon-plasmon coupled mode in $200 \sim 600 \mathrm{~cm}^{-1}$ range. The vibration amplitude is localized mainly to carbon atom and its nearest neighbors because carbon is lighter than host atoms. The LVM intensity normalized with respect to internal reference. $I_{2}$ To. is directly proportional to the carrier concentration. This makes $I_{L, M}$ a carrier density calibrator, even though its practical used might be limited due to weakness of the $C_{\text {is }}$ LVM.

Both the LOPC mode and LO phonon are investigated in the optic-phonon regime. The LO phonon mode is attributed to coming from the depletion region near surface. The LOPC mode is due to the interaction between the plasma oscillations of the free carriers and LO phonons which are related to electric dipole moment. The lineshape of the LOPC band has been calculated by using the DP and electron-phonon interaction mechanisms. The calculated LOPC mode shape is asymmetric at low carrier concentration and more symmetric at high carrier concentration, which agrees on the observed LOPC features. The calculated LOPC Raman scattering rate also shows one mode behavior observed from high plasmon damping system.

In our measurements for very high carrier concentrations, the LOPC lineshape shows no systematic variation with carrier concentration. On the contrary, the LOPC 
mode energy shows a systematic shift in my experiments. Therefore I focused on the LOPC mode energy' because no systematic information can be draun from the lineshape analysis. Both of the LO phonon mode and LOPC mode peaks redshift between carrier concentrations of $2.3 \times 10^{19}$ and $1 \times 10^{20} \mathrm{~cm}^{-3}$ (Figures 5.t and 5.8). The LOPC peak position. for varying $p$. is calculated by solving Eq. 2.23 for the roots of dielectric function. $\varepsilon(\omega)$. The roots with fixed $\omega_{\text {TO }}$ and (1), show good agreement with the LOPC positional dependence on $p$ observed by Irmer et al. [51] for Zn doped GaAs. However. the roots with fixed $\omega_{\text {TO }}$ and (1) LO failed to explain larger LOPC mode redshift observed from Be doped GaAs [52] and carbon doped GaAs. We tentatively attribute the difference to internal strain due to the relative smallness of dopants in the GaAs crystal because the red shift of the LO band is not fully described by the influence of high hole concentrations on the dielectric function. The LOPC band red shift is adequately described by a dielectric function which includes phonon. free carrier contributions and the softening of the optic phonon branches implied by the position of the LO phonon. B: examining the relative intensities of the LOPC band and LO phonon. we find that $\mathrm{I}_{\mathrm{LOPC}} / \mathrm{I}_{\mathrm{LO}}$ is in proportion to $p$. This implies that the relatively straightforward measurement of this intensity ratio can be used to estimate the carrier concentration.

The LOPC mode intensity reduces drastically upon annealing. Higher carrier concentration samples show bigger reduction in LOPC mode intensity. This implies that carrier concentration decreases upon annealing because LOPC intensity is proportional to carrier concentration as shown in Fig. 5.3. To understand the carrier reduction process. Raman spectra are taken in the range of $150 \sim 2000 \mathrm{~cm}^{-1}$. No evidence of carbon at gallium site LVM. di-carbon center. and LVM of $\mathrm{H}-\mathrm{C}_{\mathrm{As}}$ were observed in Raman spectra 
taken in this study. From the peaks at $\approx 1375$ and $\approx 1600 \mathrm{~cm}^{-1}$ from all the annealed samples, it is believed that carbon precipitates into a nanocrystalline graphite phase upon annealing. 


\section{REFERENCES}

[1] R.F. Pierret. Semiconductor Device Fundamentals. Addison-Wesley Publishing Co., New York, 1996.

[2] R. Klauder. Ann. Phys. 14:43, 1961.

[3] J. Serre and A. Ghazali. Phys. Rev. B, 28:4704, 1983.

[4] B.I. Halperin and M. Lax. Phys. Rev., 148:722, 1966.

[5] E.O. Kane. Phys. Rev., 131:790, 1961.

[6] B.E. Sernelius. Phys. Rev. B, 34:5610, 1986.

[7] P. Bhattacharya. semiconductor optoelectronic devices. Prentice Hall, New Jersey, 1994.

[8] F. Stern and R.M. Talley. Phys. Rev., 100:1638, 1955.

[9] J.I. Pankove. Optical processes in semiconductors. Dover, New York, 1971.

[10] M. Seon, M. Holtz, Ta-Ryeong Park, O. Brafman, and J. C. Bean. Phys. Rev. B, 58:4779, 1998.

[11] O. Brafman, M. A. Araújo Silva, F. Cerdeira, R. Manor, and J. C. Bean. Phys. Rev. $B, 51: 17800,1995$.

[12] P.Y. Yu and M. Cardona. Fundamentals of Semiconductors. Springer, Berlin, 1996.

[13] M. Holtz, J. C. Carty, and W. Duncan. Appl. Phys. Lett., 74:2008, 1999.

[14] M. Holtz, M. Seon, T. Prokofyeva, H. Temkin, R. Singh and Aland Chin. Appl. Phys. Lett., Submitted.

[15] M. Holtz, M. Seon, O. Brafman, R. Manor, and D. Fekete. Phys. Rev. B, 54:8714, 1996.

[16] T. Prokofyeva, T. Sauncy, M. Seon, M. Holtz, Y. Qui, S. Nikishin, and H. Temkin. Appl. Phys. Lett., 73:1409, 1998.

[17] W. Shockley. Bell. Syst. Tech. J., 28:435, 1949.

[18] H.S. Bennett. J. Appl. Phys., 60:2866, 1986. 
[19] C. Kittel. Introduction to solid state physics. 6" Ed.. John Wile! \& Sons. New York. 1986.

[20] M. Weyers, N. Putz, H. Heinecke. M. Heyen. H. Luth and P. Balk. J. Electon. Mater. 15:57. 1986.

[21] R.J. Malik, R.N. Nottenberg, E.F. Schubert, J.F. Walker. and R.W. Ryan. Appl. Phys. Lett., 53:2661, 1988.

[22] B.T. Cunningham, M.A. Haase, M.J. McCollum, J.E. Baker. and G.E. Stillman. Appl. Phys. Lett., 54:1905, 1989.

[23] M.A. Tischler. IBM J. Res. Develop. 34:828, 1990.

[24] B.T. Cunningham, M.A. Haase, M.J. McCollum. J.E. Baker and G.E. Stillman. Appl. Phys. Lett., 54:1905, 1989.

[25] M. Konagai, T. Yamada, T. Akatsuka, K. Saito, and E. Tokumitsu. J. ('rist. Growth. 98:167, 1989.

[26] C.R. Abernathy, S.J. Pearton, R. Caruso, F. Ren, and J. Kovalchik. Appl. Phys. Lett., 55:1750, 1989.

[27] B.J. Aitchison, N.M. Haegel, C.R. Abernathy, and S.J. Pearton. Appl. Phys. Lett., 56:1154, 1990.

[28] H.M. You, T.Y. Tan, U.M. Gösele, S.-T. Lee. G. Höfler. K.C. Hsieh. and N. Holonyak, Jr. J. Appl. Phys. 74:2450, 1993.

[29] A.S. Barker. Jr., and A.J. Sievers. Rev: Mod. Phis., 47:Suppl. No. 2. 1975.

[30] J.W. Allen. J. Phys. (':Solid State Phys, 3:L48, 1969.

[31] B. Pajot, R.C. Newman. R. Murray, A. Jalil. J. Chevallier. and R. Azoulay. Phys. Rev. B., 37:4188, 1988.

[32] R.S. Leigh and R.C. Newman. Semicond. Sci. Technol., 3:84, 1988.

[33] R.C. Newman. Semiconductors and Semimetals, vol. 38: Imperfections in III/V Materials. Edited by R.K. Willardson, A.C. Beer. and E.R. Weber. Academic Press, New York, 1993.

[34] K. Woodhouse, R. C. Newman, T. J. de Lyon, J. M. Woodal, G. J. Scilla, and F. Cardone. Semicond. Sci. Technol. 6:330, 1991.

[35] D.G. Thomas. J.J. Hopfield. Phys. Rel., 150:680, 1966. 
[36] J. Wagner, M. Maier, R. Murray, R.C. Newman, R.B. Beall. and J.J. Harris. J. Appl. Phys., 69:971, 1991.

[37] M. Holtz, R. Zallen, A.E. Geissberger and R.A. Sadler. J. Appl. Phys. 59:1946, 1986.

[38] T. Nakamura and T. Tatoda. J. Appl. Phys., 57:1084, 1985.

[39] J. Wagner, M. Maier, Th. Lauterbach, K.H. Bachem, A. Fischer, K. Ploog, G. Mörsch, and M. Kamp. Phys. Rev. B, 45:9120, 1992.

[40] R. Trommer and M. Cardona. Phys. Rev. B, 17:1865, 1978.

[41] M. Chandrasekar, H.R. Chandrasekar, M. Grimsditch, and M. Cardona. Phys. Rev. B., 22:4825, 1980.

[42] T.L. Chow. Classical Mechanics. John Wiley \& Sons, New York, 1995.

[43] N. W. Ashcroft and N. D. Mermin. Solid state physics. Saunders college publishing, Fort Worth, 1976.

[44] F.H. Pollak. Test and Measurement World. 5:120, 1985.

[45] R.A. Cowley and G. Dolling. Phys. Rev. Lett., 14:549, 1965.

[46] M. Born and K. Huang. Dynamical theory of crystal lattices, Oxford University Press, New York, 1954.

[47] A. Mooradian, and G.B. Wright. Phys. Rev. Lett., 16:999, 1966.

[48] K. Murase, S. Katayama, Y. Ando, and H. Kawamura. Phys. Rev. Lett., 33:1481, 1974.

[49] G. Abstreiter, R. Trommer, M. Cardona, and A. Pinczuk. Solid State Communications, 30:703, 1979.

[50] T. Yuasa and M. Ishii. Phys. Rev. B, 35:3962, 1987.

[51] G. Irmer, M. Wenzel, and J. Monecke. Phys. Rev. B, 56:9524, 1997.

[52] R. Fukasawa, K. Sakai, and S. Perkowitz. Jpn. J. Appl. Phys., 36:5543, 1997.

[53] D.T. Hon and W.L. Faust. Appl. Phys., 1:241, 1973. 
[54] G. Irmer, V.V. Toporov, B.H. Bairamov, and J. Monecke. Phys. Status Solidi B, 119:595, 1983.

[55] W. Bala, F. Firszt, M. Grinberg, M. Drozdowski, and M. Kozielski. Acta Phys. Pol. $A, 75: 75,1989$.

[56] H. Yugami, S. Nakashima, K. Sokai, H. Kojima, M. Hangyo, and A. Mitsuishi. J. Phys. Soc. Jpn., 56:1881, 1987.

[57] S. Nakashima, H. Yugami, A. Fujii, and M. Hangyo. J. Appl. Phys., 64:3067, 1988.

[58] T. Kosawa, T. Kachi, H. Kano, Y. Taga, M. Hashimoto, N. Koide, and K. Manabe. J. Appl. Phys., 75:1098, 1994.

[59] K. Wan and J.F. Young. Phys. Rev. B, 41:10772, 1990.

[60] R. Fukasawa and S. Perkowitz. Phys. Rev. B, 50:14119, 1994.

[61] M. Gargouri, B. Prevot, and C. Schwab. J. Appl. Phys., 62:3902, 1987.

[62] A. Mlayah, R. Carles, G. Landa, E. Bedel, and A. Muñoz-Yagüe. J. Appl. Phys., 69:4064, 1991.

[63] R. Fukasawa and S. Perkowitz. Jpn. J. Appl. Phys., 35:132, 1996.

[64] T. Kamijoh, A. Hashimoto, H. Takano, and M. Sakuta. J. Appl. Phys., 59:2382, 1986.

[65] K. Wan, J.F. Young, R.L.S. Devine, W.T. Moore, A.J. Spring Thorpe, C.J. Miner, and P. Mandeville. J. Appl. Phys., 63:5598, 1988.

[66] D. Olego and M. Cardona. Phys. Rev. B, 24:7217, 1981.

[67] R.A. Muñoz-Hernández, S. Jiménez-Sandoval, G.Torres-Delgado, C. Roch, X.K. Chen, and J.C. Irwin. J. Appl. Phys., 80:2388, 1996.

[68] M. Cardona and G. Güntherodt. (eds.) Light Scattering in Solids IV, Springer, Berlin, . 1984.

[69] M. Cardona. (ed.) Light Scattering in Solids I, Springer, Berlin, 1975.

[70] D.A. Long. Raman Spectroscopy, McGraw-Hill. London, 1977.

[71] C.V. Raman and K.S. Krishnan. Nature, 121:501, 1928. 
[72] R.S. Drago. Physical Methods for Chemist, Harcourt Brace Jovanovich College Publisher, Mexico City, 1977.

[73] W. Hayes and R. Loudon. Scattering of Light by Crystals, John Wiley \& Sons, New York, 1978.

[74] M.I. Nathan and G. Burns. Phys. Rev., 129:125, 1963.

[75] D. Olego and M. Cardona. Phys. Rev. B, 22:886, 1980.

[76] L. Wang, N.M. Haegel, and J.R. Lowney. Phys. Rev. B., 49:10976, 1994.

[77] G. Borghs, K. Bhattacharya, K. Deneffe, P. Van Mieghem and R. Mertens. J. Appl. Phys., 66:4381, 1989.

[78] H.D. Chen, M.S. Feng, P.A. Chen, K.C. Lin, and C.C. Wu. J. Appl. Phys., 75:2210, 1994.

[79] Z.H. Lu, M.C. Hanna, and A. Majerfeld. Appl. Phys. Lett., 64:88, 1994.

[80] S.I. Kim, M.S. Kim, S.K. Min, and C. Lee. J. Appl. Phys., 74:6128, 1993.

[81] J. Lee, I. Kim, B. Choe, W.G. Jeong, Y.K. Sin and W.S. Min. J. Appl. Phys., 79:9278, 1996.

[82] E.W. Williams and H.B. Bebb. Transport and optical phenomena, volume 8 of Semiconductors and semimetals, chapter 5, Academic Press, New York, 1972.

[83] H.B. Bebb and E.W. Williams. Transport and optical phenomena, volume 8 of Semiconductors and semimetals, chapter 4, Academic Press, New York, 1972.

[84] W.H. Louisell. Radiation and noise in quantum electronics, chapter 5, McGraw-Hill, New York, 1964.

[85] H.A. Bethe. Intermediate quantum mechanics, chapter 12, Benjamin, New York, 1964.

[86] R.K. Ahrenkiel. Monority carriers in III-V semiconductors, volume 39 of Semiconductors and semimetals, chapter 2, Academic Press, San Diego, 1993.

[87] D.R. Vij. Luminescence of solids, chapter 3, p.101, Plenum Press, New York, 1998.

[88] Y.P. Varshni. Physica, 39:149, 1967.

[89] J. S. Blakemore. J. Appl. Phys., 53:520, 1982. 
[90] J.C. Sarace, R.H. Kaiser, J.M. Whelam, R.C.C. Leite. Phys. Re'v. A. 137:623. 1965.

[91] E. Burstein. Phys. Rev, 93:632, 1954.

[92] T.S. Moss. Proc. Phys. Soc. B, 76:775, 1954.

[93] H.S. Bennett and J.R. Lowney. J. Appl. Phys., 62:521. 1987.

[94] M.I. Nathan, G. Burns, S.E. Blum, and J.C. Marinace. Phy:s. Rer.. 132:1482, 1963.

[95] T.N. Morgan. Bull. Am. Phys. Soc., 9:77. 1964.

[96] H.C. Casey and F. Stern. J. Appl. Phys, 47:631, 1976.

[97] G.E. Höfler, H.J. Höfler, N. Holonyak. Jr. and K.C. Hsieh. J. Appl. Phys. 72:5318. 1992.

[98] H. Fushimi and K. Wada. J. Appl. Phys., 82:1208, 1997.

[99] W.Y. Han, Y. Lu, H.S. Lee, M.W. Cole, S.N. Schauer, R.P. Moerkirk, K.A. Jones and L.W. Yang. Appl. Phys. Lett., 61:87, 1992.

[100] M.C. Hanna, Z.H. Lu, and A. Majerfeld. Appl. Phys. Lett., 58:164, 1991.

[101] S. Nozaki, K. Takahashi, M. Shirahama. K. Nagao, J. Shirakashi, E. Tokumitsu, and M. Konagai. Appl. Phys. Lett., 62:1913. 1993.

[102] A.J. Moll. E.E. Haller, J. W. AgerIII, and W. Walukiewicz. Appl. Phys. Lett. 65:1145, 1994.

[103] S.P. Westwater, T.J. Bullough. J. ('rys. Growth. 170:752, 1997.

[104] W.E. Hoke, P.J. Lemonias, D.G. Weir. H.T. Hendriks. and G.S. Jackson. J. Appl. Phys., 69:511, 1991.

[105] J. Wagner, R. C. Newman, B. R. Davidson, S. P. Westwater, T. J. Bullough, T. B. Joyce, C. D. Latham. R. Jones, and S. Öberg. Phys. Rev. Lett., 78:74, 1997.

[106] H. Sohn, E.R. Weber, S. Nozaki, M. Konagai, K. Takahashi. Mat. Res. Soc: Symp. Proc., 262:129, 1992.

[107] M. Uematsu, K. Wada, and U. Gösele. Appl. Phys. A. 55:301. 1992.

[108] N. Pan, S. S. Bose. M. H. Kim, G. E. Stillman, F. Chambers. G. Devane. C. R. Ito. and M. Feng. Appl. Phys. Lett., 51:596, 1987. 
[109] J. Wagner, K. H. Bachem, B. R. Davidson, R. C. Newman. T. J. Bullough, and T. B. Joyce. Phys. Rev. B, 51:4150, 1995.

[110] C.R. Abernathy, S.J. Pearton, M.O. Manasreh, D.W. Fischer, and D.N. Talwar. Appl. Phys. Lett., 57:294, 1990.

[111] S. Kim, K.S. Eom, Y. Kim, M.S. Kim, S.K. Kim, C. Lee, M.H. Kwak and D.S. Ma. J. Crystal Growth, 126:441, 1993.

[112] D.M. Szmyd, M.C. Hanna, and A. Majerfeld. J. Appl. Phys., 68:2376, 1990.

[113] S.A. Campbell. The science and engineering of microelectronic fabrication. Oxford University Press, New York, 1996.

[114] B.T. Cunningham, M.A. Haase, M.J. McCollum, J.E. Baker, and G.E. Stillman. Appl. Phys. Lett. 54:1905, 1989.

[115] G.B. Stringfellow. Organometallic vapor-phase epitaxy: Theory and practice. Academic Press, San Diego, 1989.

[116] T.F. Kuech. Materials Science Reports, 2:1, 1987.

[117] T. Sauncy. Photoluminescence studies of 100- and 111 grown indium gallium arsenide strained single quantum wells under hydrostatic pressure, Ph.D. dissertation, Texas Tech U., Lubbock, 1998.

[118] D.E. Aspnes and A.A. Studna. Phys. Rev. B, 27:985, 1983.

[119] T. Dallas. Structural phases of disordered carbon materials, Ph. D. dissertation, Texas Tech U., Lubbock, 1996.

[120] C. Adjouri, A. Elliasmine and Y. Le Duff. Spectroscopy, 11(6):44, 1996.

[121] W.D. Duncan, Private communication with W.M. Duncan. May 27, 1997.

[122] S.I. Kim, M.S. Kim, Y. Kim, K.S. Eom, S.K. Min, and C. Lee. J. Appl. Phys., 73:4703, 1993.

[123] Z.H. Lu and A. Majerfeld. J. Appl. Phys. 75:2648, 1994.

[124] A.N. Tikotov, E.I. Chal̀kina, E.M. Komora, and N.G. Ermakova. Sov. Phys. Semicond., 15:198, 1981.

[125] R.C. Miller, D.A. Kleinman, W.A. Nordland, Jr., and R.A. Logan. Phys. Rev. B, 23:4399, 1981. 
[126] D.N. Nasledov. V.V. Negreskul. and B.V. Tsarenkov. Sov. Phys. S'micond. 3:1012. 1970.

[127] S.M. Sze. Physics of semiconductor devices. Wiley. New York. 1981.

[128] P. Enquist. J. Appl. Phys., 71:704. 1992.

[129] K. Watanabe and H. Yamazaki. Appl. Phys. Lett., 59:434. 1991.

[130] E.W. Williams. Phys. Rev., 168:922. 1968.

[131] H. Kressel, F.Z. Hawrylo, M.S. Abrahams. and C.J. Buiocchi. J. Appl. Phys. 39:5139, 1968.

[132] K. Saito. E. Tokumitsu. T. Akatsuka. M. Miyauchi. T. Yamada. M. Konagai. and K. Takahashi. J. Appl. Phys. 64:3975. 1988.

[133] J.S. Lee. I. Kim, B.D. Choe, and W.G. Jeong. J. Appl. Phy'. 76:5079, 1994.

[134] T.F. Kuech, M.A. Tischler. P.-J. W'ang. G. Scilla, R. Potemski. and F. Cardone. Appl. Phy:s. Lett. 53:1317. 1988.

[135] B.T. Cunningham. J.E. Baker. J.S. Major. Jr.. N. Holonyak. Jr.. and G.E. Stillman. Appl. Phys. Lett. 55:687, 1989.

[136] H. Shen, F. Pollak, and R.N. Sacks. Appl. Phys. Lett. 47:891. 1985.

[13.7] R. Fukasawa, M. Wakaki, K. Ohta, and H. Okumura. Jpn. J. Appl. Phys. . 25:652. 1986.

[138] J. Bielmann, B. Prevot, and C. Schwab. J. Phys. ('. Solid State Phys. 16:1135. 1983.

[139] M. Cardona and G. Güntherodt. (eds.) Light Sccuttering in Solids II. Springer. Berlin, 1982.

[140] S. Ushioda, A. Pinczuk. E. Burstein. D.L. Mills. Light scattering spectra of solids, ed. by G.B. Wright, Springer. New York. 1969. p.347-358.

[141] W.D. Johnston, I.P. Kaminow. Phis. Rev.. 188:1209. 1969.

[142]O. Madelung. Data in Science and Technology: Semiconductors Group II' Elements and III-I' 'ompounds, Springer-Verlag, New York 1991.

[1+3] O.K. Kim and W.G. Spitzer. J. Appl. Phys. 50:4362. 1979. 
[144] D. Olego, M. Cardona. Phys. Rev. B, 23:6592, 1981.

[145] R. Fukasawa, S. Katayama, A. Hasegawa, and K. Ohta. J. Phys. Soc. Jpn., 57:3632. 1988.

[146] J.D. Wiley and M. DiDomenico, Jr.. Phys. Rev. B, 2:427, 1970.

[147] M. Wenzel, G. Irmer, J. Monecke, and W. Siegel. Semicond. Sci. Technol.. 13:505. 1998.

[148] H.J. Lee and D.C. Look. J. Appl. Phys., 54:4446, 1983.

[149] L.A. Borisova, A.F. Kravcenko, K.N. Kot, and E.M. Skok. Sov. Phys. Semicond, 6:693, 1972.

[150] H. Neumann and Van Nam Nguyen. Krist. Tech.. 13:211. 1978.

[15.1] K. Takeda, N. Matsumoto, A. Taguchi, H. Taki, E. Ohta, and M. Sokata. Phys. Rev. $B, 32: 1101,1985$.

[152] L. Gouskov, S. Bilac, J. Pimentel, and A. Gouskov. Solid State Electron. 28:653. 1977.

[153] F.P. Kesamanly, Yu.V. Maltsev, D.N. Nasledov, and Yu.I. Ukhanov. Phys. Stat. Sol., 13:K119, 1966.

[154] W.E. Spicer, P.W. Chye, C.M. Garner, I. Lindau and P. Pianetta. Surface Science, 86:763, 1979.

[155] P.E. Gregory, and W.E. Spicer. Phys. Rev. B, 13:725, 1976.

[156] R.S. Berg, N. Mavalvala, T. Steinberg, and F.W. Smith. J. of Electronic Materials, 19:1323, 1990.

[157] R.L. Farrow, R.K. Chang, S. Mroczkowski, and F.H. Pollak. Appl. Phys. Lett., 31:768, 1977.

[158] S.A. Stockman, A.W. Hanson, S.M. Lichtenthal, M.T. Fresina, G.E. Höfler, K.C. Hsieh, and G.E. Stillman. J. Electron. Mater., 21:1111, 1992.

[159] J.R. Dennison, M. Holtz, and G. Swain. Spectroscopy, 11 8:38, 1996.

[160] F. Tuinstra and J. L. Koenig. J. Chem. Phys., 53:1126, 1970. 